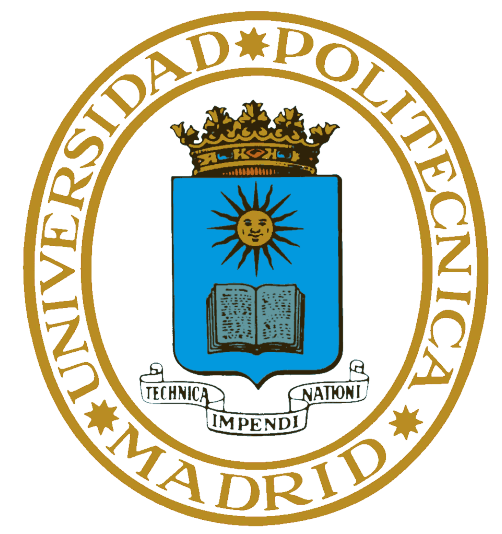

ESCUELA TÉCNICA SUPERIOR DE INGENIEROS INFORMÁTICOS

Learning secrets and models from execution time PH.D THESIS

\author{
José Vila Bausili
}

MSc in Computer Engineering 
Copyright(C)2020 by José Vila Bausili 
DEPARTAMENTAMENTO DE LENGUAJES Y SISTEMAS INFORMÁTICOS E INGENIERIA DE SOFTWARE

ESCUELA TÉCNICA SUPERIOR DE INGENIEROS INFORMÁTICOS

\title{
Learning secrets and models from execution time
}

SUBMITTED IN PARTIAL FULFILLMENT OF THE REQUIREMENTS FOR THE DEGREE OF:

Doctor of Philosophy in Software, Systems, and Computation

\author{
Author: José Vila Bausili \\ MSc in Computer Engineering \\ Advisor: Prof. Boris Köpf \\ Doctor of Sciences
}

May 2020

Thesis Committee:

Chair

Prof. Frank Piessens - KU Leuven, Belgium

Secretary

Prof. Juan Caballero - IMDEA Software Institute, Spain

Members

Dr. Clémentine Maurice - CNRS, France

Prof. Jan Reineke - Saarland University, Germany

Dr. Yuval Yarom - University of Adelaide, Australia 


\section{Abstract of the Dissertation}

In this dissertation we study some of the problems arising on computer systems that leak information through execution time. We study several instances of how these leaks can be used to both learn secrets - of a confidential computation - and models - of an underlying component-, and we provide examples that violate previous assumptions about systems' security or about the attackers' capabilities.

In particular, we study time leakage under three different scenarios, providing multiple independent contributions in each of them:

- First, we show that event-driven software systems are susceptible to sidechannel attacks. The key observation is that event loops form a resource that can be shared between mutually distrusting programs. Hence, contention of this resource by one program can be observed by the others through variations in the time the latter processes take for dispatching their events. We exploit two different shared event loops in the Chrome web browser, and use the information obtained in three different attacks: for web page fingerprinting, for keystroke detection, and for a cross-origin covert channel.

- Then, we show that our contributions are both theoretical and practical. On the theoretical side, we formalize the problem of finding minimal eviction sets, a key primitive for several microarchitectural attacks, and devise novel algorithms that improve the state-of-the-art from quadratic to linear. On the practical side, we perform a rigorous empirical analysis that exhibits the conditions under which our algorithms succeed or fail.

- Finally, we present a practical end-to-end solution for inferring deterministic cache replacement policies using off-the-shelf techniques for automata learning and program synthesis. The enabling contribution is a chain of two abstractions: a clean interface to the hardware cache replacement policies based on timing measurements on a silicon CPU; and a mapper that 
exposes a membership oracle to the cache replacement policy abstracting away the details regarding cache content management.

The results of this thesis constitute an evidence that better models and quantification methods, for both software and hardware systems, are required in order to reason about the soundness and trade-offs of security countermeasures; and provide a basis for principled countermeasures against, or paths for further improving the efficiency of, several side channel attacks. 


\section{Resumen de la Tesis Doctoral}

La presente disertación estudia los problemas surgidos en sistemas informáticos que filtran información a través del tiempo de ejecución. El trabajo se centra en cómo dichas fugas pueden ser utilizadas para aprender secretos - de computaciones confidenciales - o modelos - de los componentes subyacentes - proporcionando ejemplos que violan suposiciones previas sobre la seguridad de los sistemas o sobre los límites de un atacante.

El estudio se centra en la fuga de información a través del tiempo de ejecución en tres escenarios distintos, aportando múltiples contribuciones independientes en cada uno de ellos:

- Primero, se muestra cómo los sistemas orientados a eventos son susceptibles a ataques laterales. La observación clave es que los bucles de eventos forman un recurso compartido entre varias partes desconfiadas. De este modo, la contención de este recurso por un programa puede ser observada por otros a través de variaciones en el tiempo de procesamiento del proceso de control. En concreto, se explotan dos bucles de eventos compartidos en el navegador Chrome, y se usa la información obtenida en tres ataques distintos para: identificar paginas web, detectar pulsaciones de teclado del usuario, y transmitir información entre páginas de distinto origen.

- Después, se demuestra que las contribuciones de esta disertación son tanto teóricas como prácticas. En lo teórico, se formaliza el problema de encontrar conjuntos mínimos de desalojo en cachés, un paso clave para varios ataques microarquitecturales; y se presenta un nuevo algoritmo que mejora el estado del arte desde coste cuadrático a lineal. En la práctica, llevamos a cabo un riguroso análisis empírico que exhibe las condiciones bajo las cuales el algoritmo tienen o no éxito.

- Finalmente, se presenta una solución práctica y completa para aprender políticas de reemplazamiento de cachés deterministas utilizando técnicas de 
aprendizaje de autómatas y síntesis de programas. La contribución clave es una cadena de dos abstracciones que expone un oráculo a la política de reemplazamiento de la caché, basándose únicamente en los tiempos de acceso a memoria de la CPU.

Los resultados de esta tesis constituyen una evidencia de que se requieren mejores modelos y métodos para evaluar tanto la seguridad de los sistemas informáticos como la de las medidas contra ciberataques; y establecen una base para: definir contramedidas formales antes, y mejorar la eficiencia de, varios ataques laterales. 
"All we have to decide is what to do with the time that is given us." - Gandalf 


\section{Acknowledgments}

It is customary to thank people that helped the thesis to be performed such as the advisor, paper collaborators, family, and whoever the author would like to thank. 
It seemed funny - and I was tempted - to end this section with the previous paragraph, but that would have not been fair to all the people that have helped me in some way or another during these years.

First and foremost, I want to thank Boris Köpf, my advisor, who gave me the opportunity to do research, the freedom to seek whatever I found interesting and exciting at any time, the guidance to look at problems in a unique way, and, from time to time, the extra focus I needed to continue on track. I also have to thank Arantxa, for her patience all these years, for bearing with my healthy work-life balance, and for all her support. I suspect she will be even happier than myself for finally having written this. My parents, who have always supported me. I realize that I keep pissing you off more than I should, but I love you and I am very grateful for all the sacrifices you have made. Finally, thanks to all the people I met and learned from along the way, all the folks at the IMDEA Software Institute, and all the colleagues that in some way challenged or inspired me.

Doing a PhD was never on my plans. I started with very little idea of what research and academia were about, half intrigued by its apparent freedom and half disenchanted by my short experience in industry, almost by chance. But today, I find myself with a complete different appreciation for many topics that, without doing a doctorate, would have been very difficult for me to cultivate. Overall, I am deeply grateful for how this whole experience has woken up a profound passion for science.

When friends and family often asked me the unspoken question- "When are you graduating?" - I used to answer with a "Don't be hasty", and how I was not pursuing a title, but on a journey where my main job was to learn things, and what else should I be doing other than enjoy it? Now that this part of the journey has reached its end, I can sincerely say that I have enjoyed all the way, with all its uncertainty, frustration, excitement, and discovery. And I hope that whatever I embark on next, I will continue to be driven by this desire for learnign. 
1 Introduction $\quad \mathbf{1}$

1.1 Thesis Contributions . . . . . . . . . . . . . 6

1.2 Thesis Organization . . . . . . . . . . . . . . . . 9

2 Background $\quad \mathbf{1 0}$

2.1 Security and Architecture of Web Browsers . . . . . . . . . . . . 10

2.1.1 Same Origin Policy . . . . . . . . . . . . . . . 10

2.1.2 Overview of the Chrome Architecture . . . . . . . . . . . . 10

2.1.3 Sharing in the Renderer Processes . . . . . . . . . . . . . . 12

2.1.4 Sharing in the Host Process . . . . . . . . . . . . . . 13

2.2 A Primer on Caching and Virtual Memory . . . . . . . . . . . 13

2.2 .1 Caches . . . . . . . . . . . . . . . . . . . . . 13

2.2.2 Virtual Memory . . . . . . . . . . . . . . . . 14

3 Timing Attacks on Shared Event Loops 17

3.1 Eavesdropping on Event Loops in Chrome . . . . . . . . . . . . . 19

3.1.1 The Renderer Process Event Loop . . . . . . . . . . . . 20

3.1.1.1 Threat Scenarios . . . . . . . . . . . 20

3.1.1.2 Monitoring Techniques . . . . . . . . . . 20

3.1.1.3 Interferences . . . . . . . . . . . . . . . 21

3.1.2 The Host Process Event Loop . . . . . . . . . . . . . . . . 22

3.1.2.1 Threat Scenarios . . . . . . . . . . . . . 22

3.1.2.2 Monitoring Techniques . . . . . . . . . . . . . . . 22

3.1.2.3 Interferences . . . . . . . . . . . . . . . . . . . . . . . . . . . . .

3.1 .3 The LoopScan Tool . . . . . . . . . . . . . . . . . 24

3.2 Attacks .............................. 24

3.2.1 Page identification ........................ 24

3.2.1.1 Sample Selection . . . . . . . . . . . 25 
3.2.1.2 Data Harvesting . . . . . . . . . . . . . . 25

3.2.1.3 Classification . . . . . . . . . . . . 26

3.2.1.4 Speed-up Techniques . . . . . . . . . . . . 27

3.2.1.5 Parameter tuning . . . . . . . . . . . . . . 28

3.2.1.6 Experimental Results . . . . . . . . . . . . . 29

3.2.1.7 Threats to Validity . . . . . . . . . . . . . 31

3.2 .2 Detecting User Behavior . . . . . . . . . . . . . . . . 31

3.2.2.1 Inter-keystroke Timing Attack on Google's OAuth login form . . . . . . . . . . . . . . . 32

3.2.2.2 Experimental Evaluation . . . . . . . . . . . 33

3.2.2.3 Experimental Results . . . . . . . . . . . . . 34

3.2.2.4 Open Challenges for Recognizing User Events . . 35

3.2 .3 Covert Channel . . . . . . . . . . . . . . . . . 36

3.2.3.1 Renderer Process . . . . . . . . . . . . . . . 36

3.2.3.2 Host Process . . . . . . . . . . . . . . 37

3.3 Discussion . . . . . . . . . . . . . . . . . . . . . . . . 38

3.3.1 Beyond Chrome . . . . . . . . . . . . . . 38

3.3.2 Countermeasures . . . . . . . . . . . . . . . . 39

3.4 Related Work . . . . . . . . . . . . . . . . . . . . . . 40

4 Finding Eviction Sets $\quad 42$

4.1 Eviction Sets . . . . . . . . . . . . . . . . 44

4.1 .1 Defining Eviction Sets . . . . . . . . . . . . . 44

4.1 .2 Testing Eviction Sets . . . . . . . . . . . . . . 45

4.1.3 The Distribution of Eviction Sets . . . . . . . . . . . . 46

4.2 Algorithms for Computing Minimal Eviction Sets . . . . . . . . . 50

4.2.1 The Baseline Algorithm . . . . . . . . . . . . . 51

4.2.2 Computing Minimal Eviction Sets for a Specific Address . 52

4.2.2.1 Threshold Group Testing . . . . . . . . . . . 52

4.2.2.2 A Linear-Time Algorithm for Computing Minimal Eviction Sets . . . . . . . . . . . . 52

4.2.3 Computing Minimal Eviction Set for an Arbitrary Address 54

4.2.4 Computing Minimal Eviction Sets for Many Virtual Addresses . . . . . . . . . . . . . . . 55

4.3 Evaluation . . . . . . . . . . . . . . . . 55

4.3.1 Design of our Analysis . . . . . . . . . . . . . 56

4.3.2 Evaluating Robustness . . . . . . . . . . . . . . 57

4.3.3 Evaluating Performance . . . . . . . . . . . . 61

4.3.4 Performance in Practice . . . . . . . . . . . . . . . 63

4.3.5 Summary ................... . . . 64

4.4 A Closer Look at the Effect of Modern Cache Replacement Policies 64

4.4.1 Adaptive Replacement Policies . . . . . . . . . . . . 65

4.4.2 Evaluating the Effect of Adaptivity . . . . . . . . . . . 66 
4.5 Related Work . . . . . . . . . . . . . . . . . . . 68

5 Learning Replacement Policies from Hardware Caches $\quad 71$

5.1 Modeling Caches and Policies . . . . . . . . . . . . . . 74

5.1 .1 Replacement Policy Model . . . . . . . . . . . . . . . . 74

5.1 .2 Cache Model . . . . . . . . . . . . . . . . . . 75

5.2 PolcA: Learning Replacement Policies . . . . . . . . . . . . . . 77

5.2.1 A Primer on Automata Learning . . . . . . . . . . 77

5.2 .2 Membership Queries . . . . . . . . . . . . . . . 77

5.2 .3 Equivalence Queries . . . . . . . . . . . . . . . 80

5.2 .4 Tool Implementation . . . . . . . . . . . . . . . . . . . 80

5.3 CacheQuery: An Interface to Hardware Memory Caches . . . . 81

5.3 .1 Domain Specific Language . . . . . . . . . . . . . 81

5.3.2 Architecture . . . . . . . . . . . . . . . . . 83

5.3.3 Implementation Challenges . . . . . . . . . . . . . 84

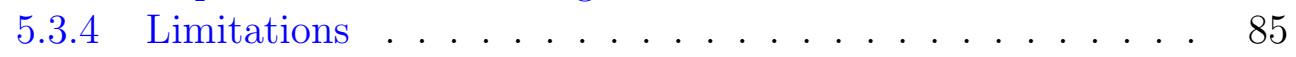

5.4 Explaining policies . . . . . . . . . . . . . . . 85

5.5 Case Study: Learning from Software-Simulated Caches . . . . . . 88

5.6 Case Study: Learning from Hardware . . . . . . . . . . . . . . . . 90

5.6 .1 Setup . . . . . . . . . . . . . . . 90

5.6 .2 Results.......................... 92

5.6 .3 Identifying Leader Sets . . . . . . . . . . . . . . . . 93

5.7 Case Study: Synthesizing Explanations . . . . . . . . . . . . . 93

5.7 .1 Setup . . . . . . . . . . . . . . . 94

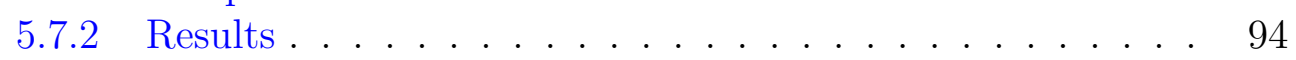

5.8 Discussion . . . . . . . . . . . . . . . . . . . . . . . . 97

5.9 Related Work . . . . . . . . . . . . . . . . . . 97

6 Conclusions \& Future Work $\quad 99$

6.1 Conclusions . . . . . . . . . . . . . . . . . . . . . . 99

6.2 Future Work . . . . . . . . . . . . . . . . . . . . . . . . . . . 99

$\begin{array}{lr}\text { Bibliography } & 102\end{array}$ 


\section{List of Figures}

1.1 Selection of abstraction layers in a modern computer: from the underlying physics (bottom), to a high-level JavaScript app running in a web browser (top). Highlights the main contributions of this thesis and their place in the hierarchy of abstractions. . . . . . .

2.1 Overview of Chrome's architecture. . . . . . . . . . . . .

2.2 Mapping from physical addresses to cache sets for Intel Skylake LLC, with 4 cores, 8 slices $(s=3), 1024$ cache sets per slice $(c=$ $10)$, lines of 64 bytes $(\ell=6)$, and associativity $a=12$. The figure also displays page offsets and frame numbers for $4 \mathrm{~KB}$ pages $(p=12)$ and $2 \mathrm{MB}$ huge pages $(p=21)$. The set index bits that are not part of the page offset determine the page color. . . . . . .

2.3 Page walk on a 64-bit system with four levels of page tables: PageMap Level 4, Page Directory Pointer, Page Directory, and Page Table for $4 \mathrm{~KB}$ pages, respectively. $2 \mathrm{MB}$ huge pages can be implemented by using a PD Entry directly as PT Entry. CPU's Control Register 3 (CR3) points to the PML4 of the running process. 15

3.1 Shared event loop. A enqueues multiple short tasks and records the time at which each of them is processed. The time difference between two consecutive tasks reveals whether $V$ has posted tasks in-between, and how long they took to execute. . . . . . . . .

3.2 Delays observed while loading different web pages, by an attacker tab sharing the renderer process. Horizontal axis depicts elapsed real time, vertical axis depicts time taken by the event loop for processing the tasks inserted by the attacker. All pages are clearly distinguishable, both by the human eye and by classification techniques. . . . . . . . . . . . . . . . . 
3.3 The path in the upper right square represents the optimal alignment between points in the time series corresponding to 'google.com' (horizontal axis) with points in the time series of 'youtube.com' (vertical axis). . . . . . . . . . . . . . . .

3.4 The top figure represents a raw trace of 200.000 time measurements from the renderer's main thread extracted while loading "google.com". The bottom figure displays the same data after being converted into a time series with $P=20 \mathrm{~ms}$, i.e. using only 250 data points. The difference in the height of the peaks is due to the accumulation of small events in the raw data, which are not perceptible in the top figure. . . . . . . . . . . . .

3.5 A global window constraint defines an envelope limiting the search space for optimal warping paths: (a) Itakura parallelogram, and (b) Sakoe-Chiba band. . . . . . . . . . . . . . . .

3.6 Web page identification performance after tuning with traces from the renderer on Linux machine (1). Effect of $P$, traceDuration, and windowSize, with three combinations of stepPattern and windowType. 29

3.7 Delay pattern generated by a keystroke in the Google OAuth login form, measured across origins on Chrome Canary v61 on OSX. The two consecutive delays of approx. 2ms each, correspond to keydown and keypress event listeners. . . . . . . . . . . .

3.8 Experimental setup for evaluating effectiveness of automatic, crossrenderer keystroke detection. . . . . . . . . . . . . .

3.9 Mouse movement captured by LoopScan tool. The graph shows 3 delays of $0.1 \mathrm{~ms}$ duration (at $t$ equals 3350,3358 and 3366), with

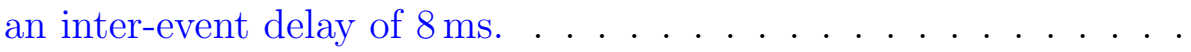

3.10 Mouse down event captured by LoopScan tool. The bottom part shows the minimap (the highlighted intervals correspond to mouse movement); the top part shows a zoomed selection. Horizontal axis depicts elapsed real time; vertical axis depicts event delays, all in milliseconds. . . . . . . . . . . . . . . . . . .

3.11 Covert channel through the I/O event loop of the Chrome's host process. Tabs in different renderer processes (one of them navigating in Incognito mode) communicate. . . . . . . . . . . . .

4.1 Eviction test for a specific address $a_{v}$ : (1) Access $a_{v}$. (2) Access $S=\left\{a_{0}, \ldots, a_{n-1}\right\}$. (3) Access $a_{v}$. If the time for (3) is larger than a threshold, then $S$ is an eviction set for $a_{v} \ldots \ldots \ldots$

4.2 Eviction test for an arbitrary address: (1) Access $S=\left\{a_{0}, \ldots, a_{n-1}\right\}$. (2) Access $S$ again. If the overall time for (2) is above a threshold, $S$ is an eviction set. . . . . . . . . . . . . . 
4.3 Robust eviction test for an arbitrary address: (1) Access $S=$ $\left\{a_{0}, \ldots, a_{n-1}\right\}$. (2) Access $S$ again, measuring access time of each element. If the access times of more than $a$ elements in (2) is above a threshold, $S$ is an eviction set. . . . . . . . . . .

4.4 Probability of random sets to be eviction sets as a function of their size, based on our theoretical models. We use $P(C)=2^{-7}$ to represent an attacker with $4 \mathrm{~KB}$ pages in the machine from Figure 2.2. The blue-circle line shows the multinomial model for an "arbitrary" eviction set. The red-cross line shows the binomial model for an "specific" eviction set. . . . . . . . . . . . . . . .

4.5 Expected number of memory accesses for finding an eviction set as a function of its size. The dashed blue line represents $P(C)=2^{-3}$, an attacker with huge pages (i.e. controls all $\gamma=10$ set index bits). The plain orange line represents $P(C)=2^{-7}$, an attacker with $4 \mathrm{~KB}$ pages (i.e. controls $\gamma=6$ ). The dotted green line represents $P(C)=2^{-13}$, an attacker w/o any control over the set index bits (i.e. $\gamma=0) . \ldots \ldots \ldots \ldots \ldots$

4.6 Skylake: Eviction for specific address $x$ on cache set zero, compared to our binomial model. Each point is the average of 1000 reductions for sets of $N$ randomly chosen addresses. . . . . . . . .

4.7 Experiments on Skylake. Each point is the average of 100 reductions for sets of $N$ randomly chosen addresses. . . . . . . . . .

4.8 Experiments on Haswell. Each point is the average of 100 reductions for sets of $N$ randomly chosen addresses. . . . . . . . . . .

4.9 The vertical green bars (left axis) depict the average number of times one needs to pick a set of addresses for finding an eviction set. Triangles (right axis) show time in seconds: blue depicts the average execution time of group test reductions; orange depicts the average execution time of baseline reductions. Different plots illustrate attackers with huge pages, $4 \mathrm{~KB}$ pages, and w/o any control over the set index bits, respectively. . . . . . . . . . .

4.10 Skylake's eviction and reduction rates per set index. With a stride of $4 \mathrm{~KB}$ and a total of 4000 addresses (most of them non-congruent). The number of sets in-between two leaders is either 32 or 98 . We rely on huge pages to precisely control the target's set index bits.

4.11 Haswell's eviction and reduction rates per set index. With a stride of $4 \mathrm{~KB}$ and a total of 4000 addresses (most of them non-congruent). 66

4.12 Eviction rate and reduction rate per set index for initial sets of 4000 partially congruent addresses. 
4.13 Skylake's total execution time per set index using backtracking and repeat-until-success. Average time over 100 samples, all of them successful. Stride of $4 \mathrm{~KB}$ (simulate adversary) and initial set of 4000 addresses (most of them non-congruent). The lowest execution times (below 0.12s), correspond to sets with higher reduction rate. Horizontal line shows the overall average execution time. . .

5.1 Leveraging PolCA and CACHEQueRY to learn a toy replacement policy of a 2-way set associative CPU cache using the LearnLib [1] framework. . . . . . . . . . . . . . . .

5.2 Transition relation for a cache $\Longrightarrow$ given that of a replacement policy $\rightarrow$. Here, cc $[\mathbf{i}]$ denotes the block stored in cc's $\mathbf{i}$-th line and $\mathrm{cc}[\mathbf{i} \mapsto \mathrm{b}]$ the cache content obtained by replacing the block in the i-th line with b. . . . . . . . . . . . . . . .

5.3 MBL syntax. We assume a given ordered set of blocks $\mathbb{B}=$ $\left\{a_{1}, a_{1}, \ldots, a_{m}\right\}$ and a value $n \in \mathbb{N}$ representing the cache's associativity such that $n<m \ldots \ldots \ldots . \ldots . \ldots . \ldots 2$

5.4 LKM's virtual file system used by CACHEQuerY . . . . . . . . . 84

5.5 Hit and miss templates using several generators. . . . . . . . . 86

5.6 Generator example defining the grammar used for the promotion rule. . . . . . . . . . . . . . . . . 87

5.7 Synthesized high-level programs for previously undocumented replacement policies using the Extended template. . . . . . . . . . 


\section{List of Tables}

3.1 List of parameters tuned for optimizing web page identification . .

3.2 10-fold cross-validation results on different machines and different event loops, with the best configuration after tuning. Machines (1) and (2) refer to the Linux desktops, (3) to the OSX laptop, as described in Section 3.2.1.2. . . . . . . . . . . . . . .

4.1 TLB implementation information for Haswell and Skylake microarchitectures. Extracted from the Intel's Architectures Optimization Manual [2]. . . . . . . . . . . . . . . . . . . . . . .

4.2 $N$ shows the optimal set sizes for different attackers $(\gamma$ bits) on Skylake $(a=12)$ using 50 time measurements per test. Time shows the average execution time of our implementations of Algorithm 1 (baseline) and Algorithm 2 (group testing) under ideal conditions.

5.1 Policy and cache alphabets (associativity $n$ ) . . . . . .

5.2 Learning policies from software-simulated caches (with 36 hours timeout). . . . . . . . . . . . . . . . . . . 89

5.3 Processors' specifications $[2,3,4] \ldots \ldots \ldots$

5.4 Results of learning policies from hardware caches. $\dagger$ indicates that the associativity has been virtually reduced using CAT. The 'Sets' column specifies the analyzed cache sets (unless otherwise specified, the findings apply to all slices). $\mathrm{F}+\mathrm{R}$ denotes the use of Flush+Refill to reset the cache set state. . . . . . . . . . . .

5.5 Synthesizing explanations for policies (of associativity 4). In the Simple template, normalize is fixed to the identity function and the grammar for expressions is simpler. In contrast, the Extended template supports the normalize rule and has a more expressive expression grammar. . . . . . . . . . . . . . . 


\section{List of Publications}

This thesis comprises three papers published at top-tier peer-reviewed academic conferences. The first of them received a distinguished paper award. The following list summarizes the aforementioned publications:

- Pepe Vila, and Boris Köpf

Loophole: Timing Attacks on Shared Event Loops in Chrome *Distinguished Paper Award*

in Proceedings of the 26th USENIX Security Symposium (CORE A*), November 2017.

- Pepe Vila, Boris Köpf, and José F. Morales Theory and Practice of Finding Eviction Sets in Proceedings of the 40th IEEE Symposium on Security and Privacy (CORE $\left.\mathrm{A}^{*}\right)$,

May 2019.

- Pepe Vila, Pierre Ganty, Marco Guarnieri, and Boris Köpf CacheQuery: Learning Replacement Policies from Hardware Caches in Proceedings of the 41th ACM SIGPLAN Conference on Programming Language Design and Implementation (CORE A*),

June 2020. 


\section{Introduction}

Computer Science is a science of abstraction - creating the right model for a problem and devising the appropriate mechanizable techniques to solve it.

A. Aho and J. Ullman

\section{Abstractions and side channels}

In software engineering, abstraction is the fundamental process for governing complexity. Similar in nature to the process of generalization, it works by hiding low level - physical, spatial, or temporal - details of a system, and by establishing well defined and simpler interfaces that allow the programmer to focus attention on more important aspects. As systems become more complex, developers end up relying in a hierarchy of abstractions. We refer to this hierarchy as the abstraction layers of the system, and illustrate an example in Figure 1.1. Abstractions are one of the main ideas that allow developers and designers to continue pushing technology forward. Namely, it is what enables programmers to write platform-independent software without having to worry about the inner physics of a transistor, the specific arrangement of logic gates, or the underlying architecture.

Unfortunately, abstractions are leaky. The classic example of this problem is traversing a two-dimensional array: as programmers, we are allowed to pretend that all the elements in the array are somewhere in a flat memory, and that the access cost to each element is uniform; however, in reality, traversing the array horizontally or vertically involves a huge performance difference, due to details that our systems abstract away, like the memory management, caching, or prefetching. This means, as per Joel Spolsky [5] words, that although abstractions 

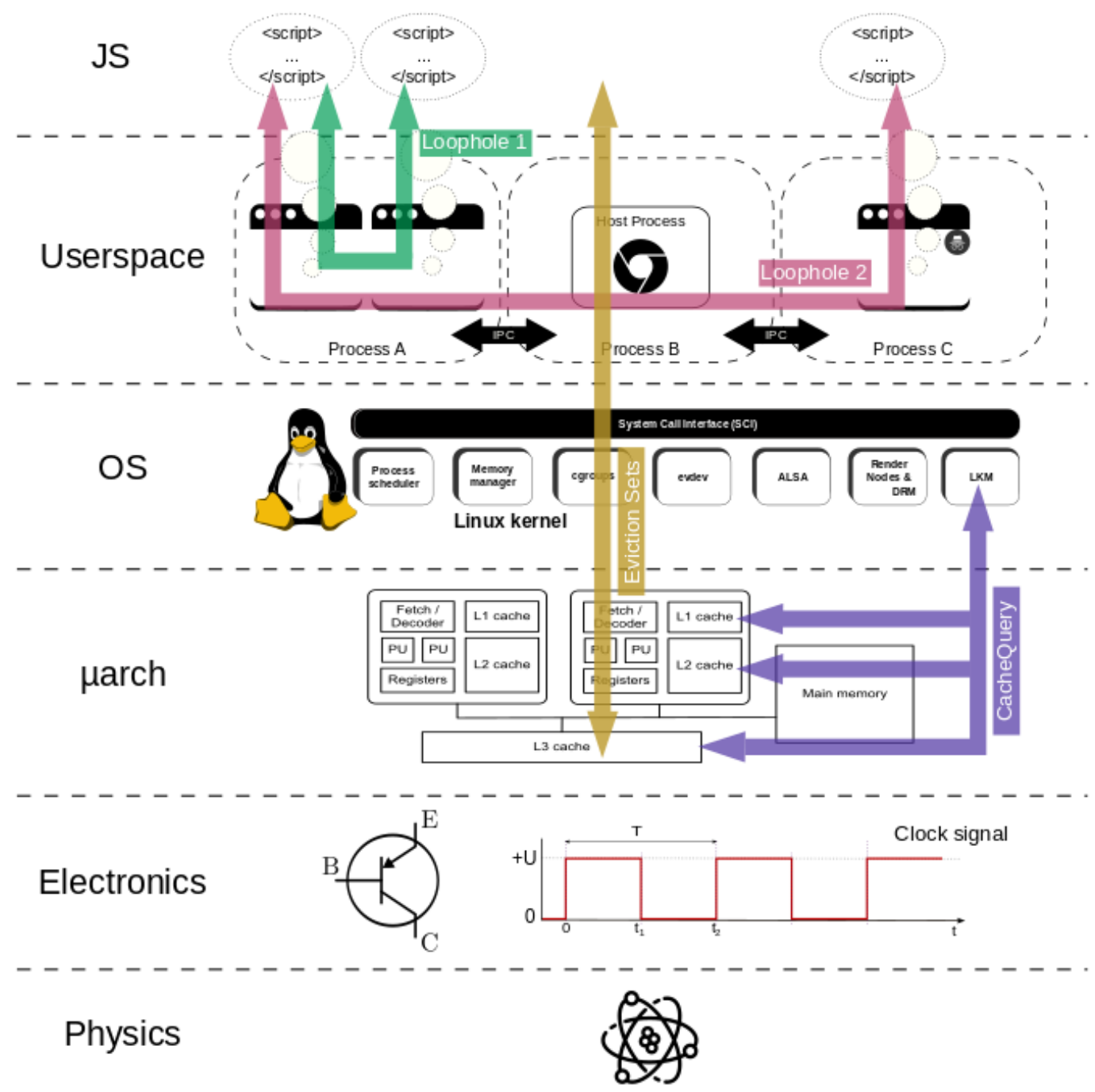

Figure 1.1: Selection of abstraction layers in a modern computer: from the underlying physics (bottom), to a high-level JavaScript app running in a web browser (top). Highlights the main contributions of this thesis and their place in the hierarchy of abstractions. 
"save us time working, they don't save us time learning". Good engineering - and specially security - , requires knowledge about the implementation details of the different abstraction layers in order to predict how leakage will affect the system.

For a long time, the main concern on leaky abstractions was about their effects on efficiency (e.g., speed or power performance), while security was swept under the rug. Now this negligence comes back to haunt us.

In the information security community, we refer to this other class of leaksthat have an effect on security - as side channels, and they occupy a central place in this thesis. Side channels are informally described as attacks based on information from the physical implementation rather than on weaknesses of the program itself. This class of attacks can be categorized in two different dimensions: the class of leakage, and the threat model. The class of leakage can be further refined based on

- the medium used, e.g., time, sound, temperature, energy, or electromagnetic radiation;

- and based on the source of leakage, e.g., control flow, cache, or memory usage.

As for the threat model, we focus on confidentiality, where we usually consider two parties - a sender and a receiver - and distinguish between

- covert-channels: where both parties - sender and receiver-cooperate in transmitting information through a channel that is not meant for communication;

- and side-channels: where an attacker - receiver-monitors the shared medium to extract information about the victim's - sender - computation, and to infer secrets (e.g., cryptographic keys, passwords, or user actions).

In this work, we leave fault attacks - which target integrity - out of scope, although some of our results are also useful to enhance software based fault attacks, like rowhammer $[6,7]$.

Side channel attacks have proven extremely useful, for example, for breaking cryptographic implementations. The first practical timing attacks against cryptographic systems were demonstrate in 1996 by Kocher [8], who was able to recover the secret keys for Diffie-Hellman, RSA, and DSS, using timing measurements. Similarly, Bernstein [9] demonstrated a complete recovery of an AES key by monitoring the cache accesses during secret-dependent table lookups. Other fundamental problems like electromagnetic emanations (or TEMPEST) have been known since 1972 [10], and have been more recently used for air-gap communication [11], spying, and breaking cryptographic implementations with power analysis [12]. 
After these seminal works, research on side-channel attacks and countermeasures has grown rapidly and across different fields, including cryptography, computer systems and architecture, programming languages, and formal methods. Ultimately, making the front page news in 2018, with the discovery of transient execution attacks $[13,14]$.

\section{Timing channels}

In this thesis, we specially focus on channels based on time measurements. The reasons for this are twofold:

- Software attacker and implicit clocks. First, in contrast to other side channels, like electromagnetic emanation, time is easily measurable from software ${ }^{1}$. It does not require a physical attacker. The only requirement is having software running on the target system, which in modern systems, like mobile or web platforms, is highly practical. Furthermore, restricting access to a precise clock has proven an arduous - if not impossibletask $[15,16,17]$. Even without explicit access to a timing API, almost any source of events can be converted into a high-resolution clock in a similar way as the oscillations of a pendulum can count seconds. For example, the number of iterations in a busy-wait loop between the start and end of a specific operation can serve as a time metric.

- Remote attacker. Second, while the capacity of most physical channels quickly degrades with distance - signal-to-noise decreases-, time remains practically the same for any fixed frame of reference. This fact enables remote timing attacks over the network $[18,19]$, even under the presence of noise.

Moreover, time does not only provide information about the duration of a secret computation, but it can also be leveraged to observe new, higher bandwidth, channels. An specially interesting class are contention based channels on shared resources. Contention [20] can be a consequence of an arbitration mechanism: when several processes request a resource at the same time we call these bandwidth resources; or when one process modifies the state of a shared resource, impacting the performance of another process, we call these storage resources. Note that execution time can be also affected by other mediums, allowing to indirectly observe them. For instance, a secret computation might affect the room temperature, which might affect the frequency of the computer, which might be measured through time.

Cache memories provide a classical example of a contention based channelthat transmits signals over time rather tan over space. In most systems, the cache

\footnotetext{
${ }^{1}$ Some systems include hardware sensors and provide APIs to access other physical medium from software, however, these are explicit channels that can be easily restricted.
} 
is fully transparent to the programmer-i.e., the Instruction Set Architecture's memory model abstracts it. However, when caches are shared among several running tasks on the system, its usage by one party can affect the performance of another. The abstraction hence leaks, opening a side channel. While it is not possible to directly ${ }^{2}$ observe or interact with the cache, time leaks become the perfect lens to look at them.

If the duration of a computation only gives coarse grained information (i.e., a time-based attacker). Using time, we can look at the cache state before and after the computation runs (i.e., an access-based attacker), what provides more fine grained information, for example, the subset of memory addresses accessed by the victim. In some cases, it is even possible to obtain a complete time seriesalso called trace - of the memory accesses during the whole computation (i.e., a trace-based attacker), rather than a single before-after picture.

\section{Topics of this thesis}

In this thesis we address three different types of questions. The first is related to exploitation, e.g., How can an attacker leverage a timing leak to break confidentiality? The second is related to quantification, e.g., What is the cost of breaking an abstraction?. The last is related to models, e.g., How does the underlying system, hidden by layers of abstractions, work?.

We approach all these questions with help of several techniques. For example, for exploitation we analyze time series using Dynamic Time Warping (DTW); for quantification we bound the adversary's capabilities by linking the problem to threshold group-testing; and finally, for modeling, we automatically learn models by using off-the-shelf automata learning and program synthesis tools.

While the questions addressed are not fundamentally novel, we approach them on new scenarios or from a different angle in comparison to prior work, which allow us to obtain interesting and novel results.

We now motivate each of these questions before summarizing the main contributionsof this thesis.

Exploitation Exploring new attacks and threats, discovering subtle implementation bugs, breaking established assumptions, and providing working proofs-ofconcept, are all key steps towards improving the security of computer systems. On one hand, this class of research directly contributes to a better understanding of how systems really work. On the other, it pressures vendors to address issues faster than they normally would. A perfect example of it is the impact of transient execution attacks, which has driven several industries to re-evaluate their security priorities.

\footnotetext{
${ }^{2}$ Performance counters in some systems provide an exception, but these often require special privileges.
} 
Quantification Most side channels arise from efficiency savings (e.g., resource sharing, speculation, or compiler optimizations), thus, it is commonly considered that securing systems require a trade-off in efficiency (or performance). However, trade-offs are not binary decisions, in order to reason about them we need mechanisms to quantify both efficiency and security. The former is usually measured in execution time, power consumption, surface occupancy, or cost, but for the latter we still lack standard tools. Instead, we often rely on adhoc definitions of security that depend on the specific environment and threat model, which makes comparison and evaluation of mechanisms difficult or impossible. A more formal approach can be, for instance, to leverage information theoretical notions to bound the amount of leakage of a system, or to use complexity, as in cryptography, to compute the cost of an attacker for achieving a certain goal. While these definitions do not provide a complete security measure by themselves, they are precise and measurable. This line of work is critical to better understand the security of our systems.

Models To leverage a time difference into a full practical attack or to propose a sound countermeasure, it becomes fundamental to understand the source of leakage. This understanding corresponds to having models of the underlying system - what is happening below all the abstraction layers. These models can be mental or formal, partial or complete, but they are necessary in order to reason across abstractions. Offensive security research, granted by the weakest link principle, rarely requires complete formal models in order to implement complex side-channel attacks. However, while not critical, more accurate models usually translate into higher capacity channels, which lead to finer grained observations, and at the end to more efficient attacks. On the contrary, on the defender's side, proposing countermeasures with only partial models or without complete understanding of the system and the attacker's capabilities (see previous point on quantification), often leads to suboptimal mechanisms that can be easily bypassed - these are so called "well-intentioned, but short-sighted mitigations" [21]. In both cases, researchers end up investing lots of resources in reverse engineering tasks with the aim of better understanding the systems and of uncovering new vulnerabilities. That is why the study of automatic techniques for inferring models from black-box systems is, at least while we lack more open designs, a keystone for security research.

\subsection{Thesis Contributions}

This thesis presents three independent contributions, each of them on a concrete scenario involving different abstraction layers. Figure 1.1 provides an overview of all of them, showing where each contribution resides in the abstraction hierarchy. 


\section{Timing attacks on shared event loops}

Event-driven programming (EDP) is the prevalent paradigm for graphical user interfaces, web clients, and it is rapidly gaining importance for server-side and network programming. Central components of EDP are event loops, which act as FIFO queues that are used by processes to store and dispatch messages received from other processes.

We demonstrate that shared event loops are vulnerable to side-channel attacks, where a spy process monitors the loop usage pattern of other processes by enqueueing events and measuring the time it takes for them to be dispatched. Specifically, we exhibit attacks against the two central event loops in Google's Chrome web browser: that of the I/O thread of the host process, which multiplexes all network events and user actions, and that of the main thread of the renderer processes, which handles rendering and Javascript tasks.

For each of these loops, we show how the usage pattern can be monitored with high resolution and low overhead, and how this can be abused for malicious purposes, such as web page identification, user behavior detection, and covert communication.

\section{Finding eviction sets}

Many micro-architectural attacks rely on the capability of an attacker to efficiently find small eviction sets: groups of virtual addresses that map to the same cache set. This capability has become a decisive primitive for cache side-channel, rowhammer, and speculative execution attacks. Despite their importance, algorithms for finding small eviction sets have not been systematically studied in the literature.

We perform such a systematic study. We begin by formalizing the problem and analyzing the probability that a set of random virtual addresses is an eviction set. We then present novel algorithms, based on ideas from threshold group testing, that reduce random eviction sets to their minimal core in linear time, improving over the quadratic state-of-the-art.

We complement the theoretical analysis of our algorithms with a rigorous empirical evaluation in which we identify and isolate factors that affect their reliability in practice, such as adaptive cache replacement strategies and TLB thrashing. Our results indicate that our algorithms enable finding small eviction sets much faster than before, and under conditions where this was previously deemed impractical.

\section{Learning replacement policies from hardware caches}

We show how to infer deterministic cache replacement policies using off-the-shelf automata learning and program synthesis techniques. For this, we construct and chain two abstractions that expose the cache replacement policy of any set in the 
cache hierarchy as a membership oracle to the learning algorithm, based on timing measurements on a silicon CPU. Our experiments demonstrate an advantage in scope and scalability over prior art and uncover 2 previously undocumented cache replacement policies.

\section{Tooling}

In order to facilitate the reproducibility of all the results, we accompany each individual contribution with its corresponding open-source implementations and datasets.

\section{LoopScan}

We implemented a JS/HTML monitoring tool for observing, in real time, shared event loops in the web browser. We use D3.js for the graphics, and provide support for 2 different event loops using 4 different techniques. Repository: https://github.com/cgvwzq/loopscan

Our page fingerprinting evaluation is done with the $\mathrm{R}$ language, for which we implemented a small library that is publicly available:

- R script for page fingerprinting: https://github.com/cgvwzq/rlang-loophole

- Dataset: https://software.imdea.org/cloud/index.php/s/fn9xduGKagwDK1Y

\section{EvSets}

To evaluate our results we implemented a $\mathrm{C}$ command line tool that allows to find minimal eviction sets using different algorithms and settings.

- EvSet's repository: https://github.com/cgvwzq/evsets

Furthermore, the repository includes a folder with the a reliable public implementation of eviction set finding for web browsers, using JavaScript and WebAssembly.

\section{CacheQuery and Polca}

CacheQuery consists on a Linux Kernel Module implementing a hardware interface to cache sets, and a Python interface providing a REPL environment for performing queries. The repository contains further instructions for installation and configuration.

Polca is our Java tool for learning automata models for cache replacement policies build on top of the LearnLib framework version 0.14.0. It is ready to use from command line with both software simulated caches and CacheQuery. 
- CacheQuery's repository: https://github.com/cgvwzq/cachequery

- Polca's repository: https://github.com/cgvwzq/polca

Besides the learning tool, Polca's repository also contains all the scripts and templates used for program synthesis, and the learned automata models.

\subsection{Thesis Organization}

This thesis is organized in six chapters: an introduction to the problem under study and its context, the necessary background and terminology used thorough the thesis, three technical contributions published in top tier conferences, and some conclusions.

Chapter 2 introduces the necessary knowledge and terminology for the rest of the thesis. We first provide a quick introduction to the security policies of modern web browsers and describe their multi-process architecture; we then introduce how cache memories are organized and how virtual memory works.

Chapter 3 contains our first technical contribution, namely, our work on timing attacks on shared event loops. We first show how to eavesdrop on different event loops of the Chrome web browser; we then present and evaluated three different attacks; and we conclude with a discussion about the implications of our work, and the effect of possible countermeasures.

Chapter 4 contains our work on finding minimal eviction sets. We provide the first formal definition of the problem and present the primitives available to an attacker; then, we present our novel algorithm for efficiently finding minimal eviction sets, inspired in threshold group testing; and we conclude with a discussion of our implementation and an exhaustive practical evaluation.

Chapter 5 contains our latest contribution for automatically learning cache replacement policies from hardware. We first present a parametric model for cache replacement policies; we then explain our automata learning approach; we present CacheQuery, a clean and deterministic interface to hardware memory caches, and its associated domain specific language; we explain how to obtain high-level descriptions with program synthesis; and we conclude with an exhaustive empirical evaluation.

Each technical chapter is accompanied by a specific section with all the related work.

Finally, Chapter 6 presents the conclusions of this thesis and discuss some future work ideas. 


\section{Background}

This chapters introduces all the necessary knowledge and terminology used thorough the thesis. We first provide a quick introduction to the security of modern web browsers; and then introduce how caches and virtual memory work.

\subsection{Security and Architecture of Web Browsers}

In this section we revisit the same origin policy and its variants, which are necessary to understand Chapter 3. We then discuss the relationship of these policies with the Chrome multi-process architecture, placing special emphasis on how resources are shared.

\subsubsection{Same Origin Policy}

The Same-Origin Policy (SOP) is a central concept in the web security model: The policy restricts scripts on a web page to access data from another page if their origins differ. Two pages have the same origin if protocol, port and host are equal.

The demand for flexible cross-origin communication has triggered the introduction of features such as domain relaxation, the postMessage API, Cross-origin Resource Sharing (CORS), Channel Messaging, Suborigins, or the Fetch API. This feature creep comes with an increase in browser complexity and attack surface, which has motivated browser vendors to move towards more robust multiprocess architectures.

\subsubsection{Overview of the Chrome Architecture}

The Chrome architecture is segmented into different operating system processes. The rationale for this segmentation is twofold: to isolate web content from the 
host [22], and to support the enforcement of origin policies by means of the OS [23]. For achieving this segmentation, Chrome relies on two processes:

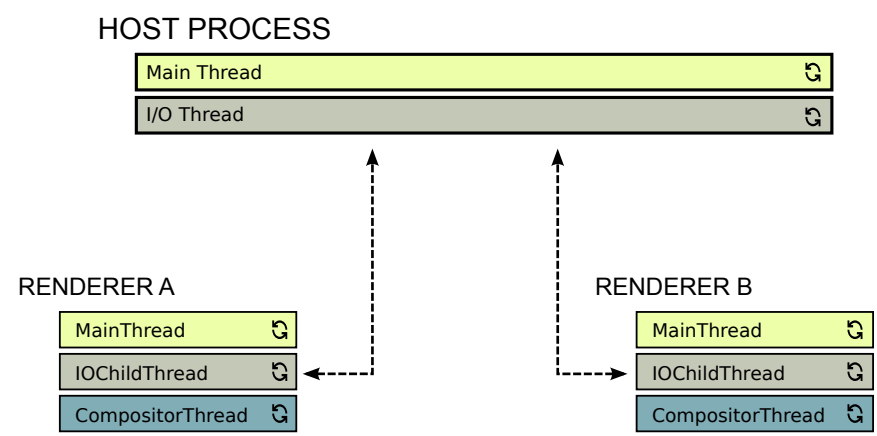

Figure 2.1: Overview of Chrome's architecture.

The host process runs the top-level browser window. It has access to system resources such as network, file system, UI events, etc., which it manages on behalf of the unprivileged renderer processes. The host process runs several threads; the most relevant ones are:

- the CrBrowserMain thread, which handles, e.g., user interaction events, and

- the IOThread, which handles, e.g., IPC, network stack, and file system.

The host process also stores all cookies in a global cookie jar. This makes stateful navigation across origins possible and provides the Internet's linkability capabilities, but it also introduces several privacy and security issues, see Section 5.9. To amend that, Chrome provides the Incognito (or private) navigation mode, which enables a temporary and independent cookie jar.

The renderer processes are sandboxed processes responsible for parsing, rendering and Javascript execution. Communication with the host process is done via an inter-process communication (IPC) system based on message passing. Each renderer runs several threads; the most relevant ones are:

- the MainThread where resource parsing, style calculation, layout, painting and non-worker Javascript runs,

- the IOChildThread, which handles IPC communication with the host process, and

- the CompositorThread, which improves responsiveness during the rendering phase by allowing the user to scroll and see animations while the main thread is busy, thanks to a snapshot of the page's state.

Each of the threads in the host and renderer processes maintains at least one event loop that is largely a FIFO queue. Inter-thread and inter-process 
communication are carried out via message passing through these queues. We next discuss scenarios where pages of different origin can share the event loops of host and renderer processes. In Section 3.1 we show how this sharing can be exploited for eavesdropping.

\subsubsection{Sharing in the Renderer Processes}

Chrome supports different policies that govern how web applications are mapped to renderer processes, and that influence whether or not event loops are shared.

The default policy is called process-per-site-instance. It requires using a dedicated renderer process for each instance of a site. Here, a site is defined as a registered domain plus a scheme. For example, https://docs.google.com and https://mail.google.com:8080 are from the same site - but not from the same origin, as they differ in subdomain and port. A site instance is a collection of pages from the same site that can obtain references to each other (e.g., one page opened the other in a new window using Javascript).

The other supported policies are more permissive. For example, the processper-site policy groups all instances of a site in the same renderer process, trading robustness for a lower memory overhead. The process-per-tab policy dedicates one renderer process to each group of script-connected tabs. Finally, the singleprocess policy lets both the host and renderer run within a single OS process (only used for debugging purposes).

Even in the restrictive default process-per-site-instance policy, there are some situations that force Chrome to host documents from different sites in the same renderer process, causing them to share the event loop:

- Iframes are currently hosted in the same process as their parent.

- Renderer-initiated navigations such as link clicks, form submissions, and scripted redirections will reuse the same renderer as the origin page.

- When the number of renderer processes exceeds a certain threshold, Chrome starts to reuse existing renderers instead of creating new ones.

On (64-bit) OSX and Linux, the threshold for reusing renderers is calculated by splitting half of the physical RAM among the renderers, under the assumption that each consumes $60 \mathrm{MB} .{ }^{1}$ In our experiments, on a machine with $4 \mathrm{~GB}$ of RAM we could spawn 31 new tabs before any renderer was shared, whereas on a machine with $8 \mathrm{~GB}$ of RAM we observed a threshold of approximately 70 renderers. There is no apparent grouping policy for the pages that can share a process when this threshold is exceeded, except for tabs in Incognito mode not being mixed up with "normal" tabs. In particular, we do not observe any preference for similar origins, same sites, or secure versus insecure pages. In fact, even filesystem pages (loaded with file://) can co-reside with an arbitrary HTTP site.

\footnotetext{
${ }^{1}$ On Android there is no threshold since the OS suspends idle processes.
} 


\subsubsection{Sharing in the Host Process}

The Chrome sandbox restricts access of renderers to privileged actions. In particular, renderers have to communicate with the host process for network requests or user input. The corresponding messages of all renderers pass through the event loop of the host process' I/O thread.

We illustrate this communication using two different examples: how user actions flow from the host to the corresponding renderer process, and conversely, how network requests flow from a renderer to the host process.

- UI flow: User actions such as mouse movements or clicks enter the browser through the main thread of the host process. The host main thread communicates the user event to the corresponding renderer by message passing between their I/O event loops, and the render acknowledges the receipt of this message. Even events with no Javascript listeners occupy the event loop of the renderer's main thread for a measurable interval.

- Net stack: Chrome's net stack is a complex cross-platform network abstraction. Any network request by a renderer is passed to the I/O thread of the host process, which forwards it to a global resource dispatcher that will pass it to a worker to fulfill the request. This worker will open a connection, if necessary, and request the resource. After the request is done, the response headers are received and sent back to the renderer process, which will respond with an ACK after reading, Finally, the body is received and the corresponding callbacks are triggered.

\subsection{A Primer on Caching and Virtual Memory}

In this section we provide the necessary background on hardware cache memories and virtual memory, and introduce the notation that will be used along Chapters 4-5.

\subsubsection{Caches}

Caches are fast but small memories that bridge the latency gap between the CPU and main memory. To profit from spatial locality and to reduce management overhead, main memory is logically partitioned into a set of blocks. Each block is cached as a whole in a cache line of the same size. When accessing a block, the cache logic has to determine whether the block is stored in the cache (a cache hit) or not (a cache miss). For this purpose, caches are partitioned into equally sized cache sets. The size or number of lines of cache sets is called associativity $a$ (or ways) of the cache. 
Cache Replacement Policies Since the cache is much smaller than main memory, a replacement policy must decide which memory block to evict upon a cache miss. Traditional replacement policies include least-recently used (LRU), pseudo-LRU (PLRU), and first-in first-out (FIFO). In modern microarchitectures, replacement policies are often more complex and generally not documented. For example, recent Intel CPUs rely on replacement policies [24, 25] that dynamically adapt to the workload.

Cache Hierarchies Modern CPU caches are organized in multiple levels, with small and fast lower-level caches per CPU core, and a larger but slower last-level cache (LLC) that is shared among different cores. The relationship between the content of different cache levels is governed by an inclusion policy. Intel caches, for instance, are usually inclusive. This means that the content of higher level caches (L1 and L2) is always a subset of the LLC's. In particular, blocks that are evicted from the LLC are also invalidated in higher levels. In this work we focus on inclusive LLCs.

Mapping Memory Blocks to Cache Sets The mapping of main memory content to the cache sets of a LLC is determined by the content's physical address. For describing this mapping, consider an architecture with $n$-bit physical addresses, cache lines of $2^{\ell}$ bytes, and $2^{c}$ cache sets. The least significant $\ell$ bits of a physical address $y=\left(b_{n-1}, \ldots, b_{0}\right)$ form the line offset that determines the position within a cache line. Bits $\left(b_{c+\ell-1}, \ldots, b_{\ell}\right)$ of $y$ are the set index bits that determine the cache set, and we denote them by $\operatorname{set}(y)$. The most significant $n-\ell-c$ bits form the $\operatorname{tag}$ of $y$. See Figure 2.2 for a visualization of the role of address bits on a Intel Skylake machine.

Cache Slicing Modern Intel CPUs partition the LLC into different $2^{s}$ many slices, typically one or two per CPU core. The slice is determined by an undocumented $s$-bit hash of the most significant $n-\ell$ bits of the address. With slicing, the $c$ set index bits only determine the cache set within each slice.

The total cache size $|M|=2^{s+c+\ell} a$ is then determined as the product of the number of slices, the number of cache sets per slice, the size of each line, and the associativity.

\subsubsection{Virtual Memory}

Virtual memory is an abstraction of the storage resources of a process that provides a linear memory space isolated from other processes and larger than the physically available resources. Operating systems, with help from the CPU's memory management unit (MMU), take care of the translation of virtual addresses to physical addresses. 


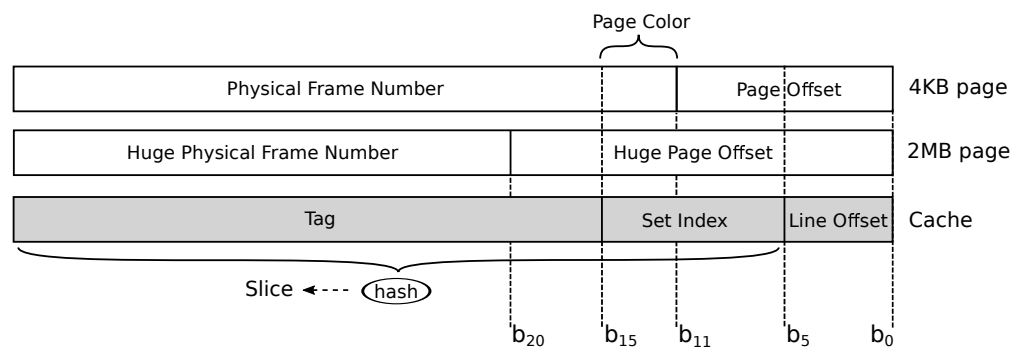

Figure 2.2: Mapping from physical addresses to cache sets for Intel Skylake LLC, with 4 cores, 8 slices $(s=3), 1024$ cache sets per slice $(c=10)$, lines of 64 bytes $(\ell=6)$, and associativity $a=12$. The figure also displays page offsets and frame numbers for $4 \mathrm{~KB}$ pages $(p=12)$ and $2 \mathrm{MB}$ huge pages $(p=21)$. The set index bits that are not part of the page offset determine the page color.

Virtual Address Translation Physical memory is partitioned in pages of size $2^{p}$. Common page sizes are $4 \mathrm{~KB}$ (i.e. $p=12$ ), or $2 \mathrm{MB}$ for huge pages (i.e. $p=21)$.

We model the translation from virtual to physical addresses as a function $p t$ that acts as the identity on the least significant $p$ bits (named page offset) of a virtual address $x=\left(x_{47}, \ldots, x_{0}\right)$. That is, the virtual and physical addresses coincide on $\left(x_{p-1}, \ldots, x_{0}\right)$. pt maps the most significant $48-p$ bits, named virtual page number (VPN), to the physical frame number (PFN). We discuss how $p t$ acts on these bits in Section 4.1.3. Figure 2.2 includes a visualization of the page offsets and physical frame numbers for small and huge pages, respectively.

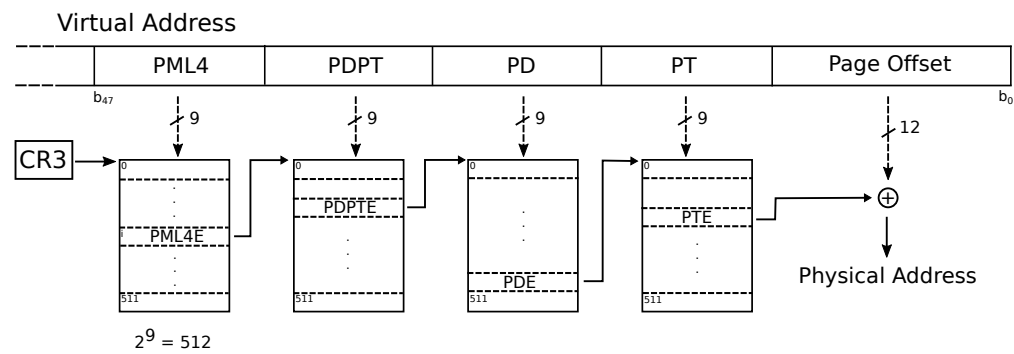

Figure 2.3: Page walk on a 64-bit system with four levels of page tables: PageMap Level 4, Page Directory Pointer, Page Directory, and Page Table for 4KB pages, respectively. $2 \mathrm{MB}$ huge pages can be implemented by using a PD Entry directly as PT Entry. CPU's Control Register 3 (CR3) points to the PML4 of the running process.

Implementing Virtual Address Translation Operating systems keep track of the virtual-to-physical mapping using a radix tree structure called page table (PT) that is capable of storing the required information efficiently. Whenever a 
virtual address is accessed, the MMU traverses the PT until it finds the corresponding physical address. This process, also known as a page walk, is illustrated in Figure 2.3. The bits of the VPN are divided into 9-bit indexes for each level of the PT, which can store up to 512 entries (of 8 bytes each). To avoid performing a page walk for each memory access, each CPU core has a translation lookaside buffer (TLB) that stores the most recent translations. A page walk only occurs after a TLB miss.

Huge Pages Modern operating systems implement support for large buffers of virtual memory to be mapped into contiguous physical chunks of $2 \mathrm{MB}$ (or 1GB), instead that of regular $4 \mathrm{~KB}$. These large chunks are called huge pages. On one hand, huge pages save page walks when traversing arrays of more than $4 \mathrm{~KB}$, improving performance. On the other hand, they increase the risk of memory fragmentation, what might lead to wasting resources.

On Linux systems, huge pages can be demanded explicitly or implicitly:

- Explicit requests are done by passing special flags to the allocation routine (e.g. flag MAP_HUGETLB to the mmap function). In order to satisfy these requests, the OS pre-allocates a pool of physical huge pages of configurable size (which by default is 0 in most systems).

- Implicit requests are referred as transparent huge pages [26]. THPs are implement with a kernel thread that, similarly to a garbage collector, periodically searches for enough contiguous $4 \mathrm{~KB}$ virtual pages that can be re-mapped into a free $2 \mathrm{MB}$ chunk of contiguous physical memory (reducing PTs size). THP can be configured as: always, meaning that all memory allocations can be re-mapped; never, for disabling it; and madvise, where the programmer needs to signal preference for being re-mapped via some flags (note that this is not a guarantee).

On other systems huge pages are implement differently, but the effect is generally the same. For instance, BSD's documentation refers to them as super pages, while Windows calls them large pages.

Interestingly, memory allocation in modern browsers is not backed by huge pages unless the system is configured with THP set to always. Hence, relying on them for finding eviction sets is not possible in most default systems. 


\section{Timing Attacks on Shared Event Loops}

Event-driven programming (EDP) consists of defining responses to events such as user actions, I/O signals, or messages from other programs. EDP is the prevalent programming paradigm for graphical user interfaces, web clients, and it is rapidly gaining importance for server-side and network programming. For instance, the HTML5 standard [27] mandates that user agents be implemented using EDP, similarly, Node.js, memcached, and Nginx, also rely on EDP.

In EDP, each program has an event loop which consists of a FIFO queue and a control process (or thread) that listens to events. Events that arrive are pushed into the queue and are sequentially dispatched by the control process according to a FIFO policy. A key feature of EDP is that high-latency (or blocking) operations, such as database or network requests, can be handled asynchronously: They appear in the queue only as events signaling start and completion, whereas the blocking operation itself is handled elsewhere. In this way EDP achieves the responsiveness and fine-grained concurrency required for modern user interfaces and network servers, without burdening programmers with explicit concurrency control.

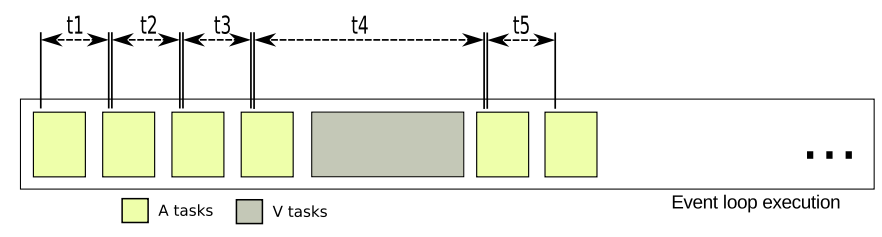

Figure 3.1: Shared event loop. A enqueues multiple short tasks and records the time at which each of them is processed. The time difference between two consecutive tasks reveals whether $V$ has posted tasks in-between, and how long they took to execute.

In this work we show that EDP-based systems are susceptible to side-channel attacks. The key observation is that event loops form a resource that can be shared between mutually distrusting programs. Hence, contention of this resource 
by one program can be observed by the others through variations in the time the control process takes for dispatching their events. Figure 3.1 illustrates such a scenario for a loop that is shared between an attacker $A$ and a victim $V$.

Attacks based on observable contention of shared resources have a long history [28] and an active present [29, 30, 31]; however, attacks against shared event loops have so far only been considered from a theoretical point of view [32]. Here, we perform the first attacks against real EDP-based systems. Specifically, we target shared event loops in the two central processes of Google's Chrome web browser: The host process, whose event loop is shared between all requests for common resources, such as network and user interface; and the renderer processes, whose loops can be shared between Javascript tasks of different tabs or iframes.

We build infrastructure that enables us to spy on both loops from a malicious HTML page. This is facilitated by the asynchronous programming model used in both Chrome and Javascript. Asynchronous function calls trigger new tasks that are appended to the same queue, in contrast to synchronous calls which are simply pushed onto the current task's call stack and executed without preemption, blocking the loop.

- For the event loop of the renderer we rely on the postMessage API, which is a Javascript feature for cross-window communication based on asynchronous callbacks. By posting messages to ourselves we can monitor the event loop with a resolution of $25 \mu \mathrm{s}$, with only one task in the loop at each point in time.

- For the event loop of the host process we rely on two different mechanisms: network requests to non-routable IP addresses, which enter the loop and abort very quickly, providing a resolution of $500 \mu \mathrm{s}$; and SharedWorkers, whose messages pass through the event loop of the host process, providing a resolution of $100 \mu \mathrm{s}$.

We use the information obtained using these techniques in three different attacks:

1. We show how event delays during the loading phase, corresponding to resource requests, parsing, rendering and Javascript execution, can be used to uniquely identify a web page. Figure 3.2 visualizes this effect using three representative web pages. While this attack shares the goal with the Memento attack [33], the channels are quite different: First, in contrast to Memento, we find that the relative ordering of events is necessary for successful classification, which motivates the use of dynamic time warping as a distance measure. Second, we show that page identification through the event loop requires only minimal training: we achieve recognition rates of up to $75 \%$ and $23 \%$ for the event loops of the renderer and host processes, respectively, for 500 main pages from Alexa's Top sites. These rates are obtained using only one sample of each page for the 
training phase.

2. We illustrate how user actions in cross-origin pages can be detected based on the delays they introduce in the event loop. In particular, we mount an attack against Google OAuth login forms, in which we measure the time between keystrokes while the user is typing a password. The timing measurements we obtain from the event loop are significantly less noisy or require less privileges than from other channels [34, 35, 36].
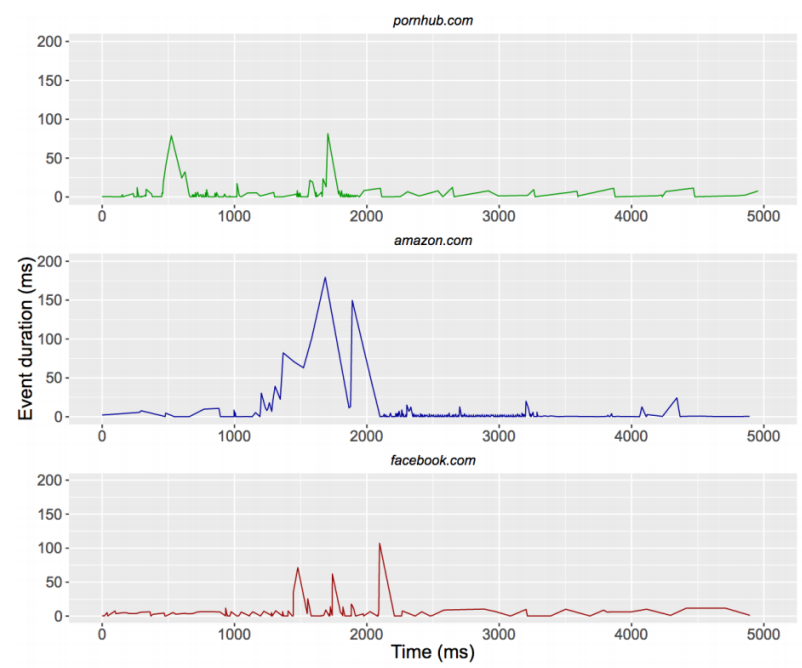

Figure 3.2: Delays observed while loading different web pages, by an attacker tab sharing the renderer process. Horizontal axis depicts elapsed real time, vertical axis depicts time taken by the event loop for processing the tasks inserted by the attacker. All pages are clearly distinguishable, both by the human eye and by classification techniques.

3. We demonstrate that shared event loops can be used to transmit information between cross-origin pages. Specifically, we implement a covert channel with a bandwidth of $200 \mathrm{bit} / \mathrm{s}$ through the renderer's main thread event loop, and another one working cross-processes of $5 \mathrm{bit} / \mathrm{s}$.

Our attacks show that event loops can be successfully spied on even with simple means. They work under the assumption that event loops behave as FIFO queues; in reality, however, Chrome's event loop has a more sophisticated structure, relying on multiple queues and a policy-based scheduler. We believe that this structure can be leveraged for much more powerful attacks in the future.

\subsection{Eavesdropping on Event Loops in Chrome}

In this section we describe how to violate the SOP by eavesdropping on the event loops of Chrome's host and renderer processes. For each of these processes, we 
describe potential threat scenarios and present a simple HTML page executing Javascript that can be used for spying. We then present our monitoring tool to visualize the event loops of the browser.

\subsubsection{The Renderer Process Event Loop}

\subsubsection{Threat Scenarios}

There are several scenarios in which an adversary site $A$ can share the event loop of the renderer's main thread with a victim site $V$. These scenarios are based on Chrome's policy for mapping sites to renderers, see Section 2.1.3. We give two examples:

- Malicious advertisement. In this scenario, $A$ runs as an advertisement iframed in $V$. The SOP protects $V$ 's privacy and integrity by logically isolating both execution environments. However, A's iframe is able to execute Javascript on $V$ 's event loop, enabling it to gather information about the user behavior in $V$.

- Keylogger. In this scenario, $A$ pops up a login form to authenticate its users via $V$ 's OAuth. Because the operation does not ask for special privileges and the password is never sent to $A$, the victim could trust it and fill the form. Meanwhile, $A$ 's page monitors keystroke timings (see Section 3.2.2), which can be used for recovering user passwords [37].

\subsubsection{Monitoring Techniques}

To monitor the renderer's event loop it is sufficient to continuously post asynchronous tasks and measure the time interval between subsequent pairs of events. We measure the monitoring resolution in terms of the interval between two subsequent measurement events on an otherwise empty loop.

The most common way of posting asynchronous tasks programmatically in Javascript is setTimeout. However, the resolution can be more than $1000 \mathrm{~ms}$ for inactive tabs, rendering this approach useless for the purpose of spying. To increase the resolution, we instead use the postMessage API for sending asynchronous messages to ourselves.

The code in Listing 3.1 shows how this is achieved. The call to performance.now() in line 2 of the function loop returns a high-resolution timestamp that is saved as described below. The call to self . postmessage $(0$, ' $*$ ') in line 3 posts message " 0 " into the renderer's event loop, where the second argument "*" indicates no restriction on the receiver's origin. Line 5 registers the function loop as an event listener, which enables it to receive the messages it has posted. This causes loop to recursively post tasks, while keeping the render responsive since other events are still being processed. 


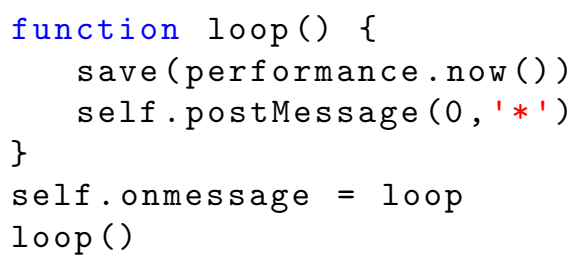

Listing 3.1: Javascript code to monitor the main thread's event loop with the postMessage API.

In order to minimize the noise introduced by the measurement script itself, the function save in line 2 uses a pre-allocated typed array (Float64Array) to store all the timing measurements. Contrary to normal Javascript's sparse arrays, typed arrays avoid memory reallocations and thus noisy garbage collection rounds, see below. With that we achieve an average delay between two consecutive tasks of around $25 \mu \mathrm{s}$ on our target machine. This resolution is sufficient to identify even short events. For example, a single mouse movement event (without explicit event listener) consumes around $100 \mu \mathrm{s}$.

\subsubsection{Interferences}

In modern browsers there are several sources of noise that affect measurement precision, beside the obvious effect of the underlying hardware platform and OS. They include:

- Just-in-time compilation (JIT). JIT can trigger code optimization or deoptimization, in the case of Chrome by the CrankShaft and Turbofan compilers, at points in time that are hard to predict. For our measurements we rely on a warm-up phase of about $150 \mathrm{~ms}$ to obtain fully optimized code.

- Garbage collection $(G C)$. In the case of V8, GC includes small collections (so-called scavenges) and major collections. Scavenges are periodical and fast $(<1 \mathrm{~ms}$ ); but major collections may take $>100 \mathrm{~ms}$, distributed into incremental steps. In our data, scavenges are easily identifiable due to their periodicity, while major collections could be spotted due to their characteristic size. On some browsers, such as Microsoft's Internet Explorer, GC rounds can be triggered programmatically, which helps to eliminate noise from the measurements enabling more precise attacks [38].

While all of these features reduce the effectiveness of our attacks, it is interesting to think of them as potential side-channels by themselves. For example, observable GC and JIT events can reveal information about a program's memory and code usage patterns, respectively [39]. 


\subsubsection{The Host Process Event Loop}

\subsubsection{Threat Scenarios}

The Chrome sandbox ensures that all of the renderer's network and user interaction events pass through the host process' I/O event loop, see Section 2.1.4. We describe two threat scenarios where this could be exploited.

- Covert channel. Pages of different origins running in different (disconnected) tabs can use the shared event loop to implement a covert channel, violating the browser's isolation mechanisms. This will work even if one (or both) pages run in incognito mode. This channel can be used for tracking users across sessions, or to exfiltrate information from suspicious web pages without network traffic.

- Fingerprinting. A tab running a rogue page of $A$ can identify which pages are being visited by the user in other tabs by spying on the shared event loop. Detecting the start of a navigation is facilitated by the fact that the I/O thread blocks for a moment when the user types in a URL and presses enter.

\subsubsection{Monitoring Techniques}

There are many ways to post asynchronous tasks into the event loop of the host process; they differ in terms of the resolution with which they enable monitoring the event loop and the overhead they imply. Below we describe two of the techniques we used.

Network Requests. The first technique is to use network requests to systematically monitor the event loop of the I/O thread of the host process. A valid network request may take seconds to complete, with only the start and end operations visible in the loop, which provides insufficient resolution for monitoring.

To increase the resolution, we make use of non-routable IP addresses. The corresponding requests enter the I/O thread's event loop, are identified as invalid within the browser, and trigger the callback without any DNS resolution or socket creation. This mechanism provides a monitoring resolution of $500 \mu \mathrm{s}$ and has the additional benefit of being independent from network noise.

Listing 3.2 shows the code of our monitoring procedure. We rely on the Javascript Fetch API for posting the network requests. The Fetch API provides an interface for fetching resources using promises, which are ideal to manage asynchronous computations thanks to their simple syntax for handling callbacks. In line 2 we request and save a high-resolution timestamp. In line 3 we request a non-routable IP address, and set the rejection callback of the promise to self, to recursively run when the request fails. 


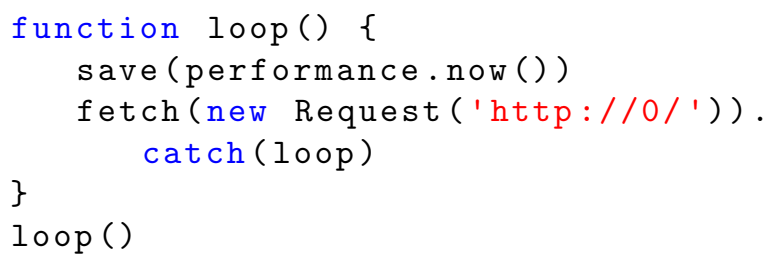

Listing 3.2: Javascript code to monitor the host's I/O thread using network requests.

Shared Workers. The second technique relies on web workers, which is a mechanism for executing Javascript in the background. Web workers that are shared between multiple pages are usually implemented in a dedicated OS process; this means they communicate via IPC and, therefore, can be used to spy on the $\mathrm{I} / \mathrm{O}$ thread of the host process. This mechanism provides a monitoring resolution of $100 \mu \mathrm{s}$. Listing 3.3 shows the code of our worker-based monitoring procedure.

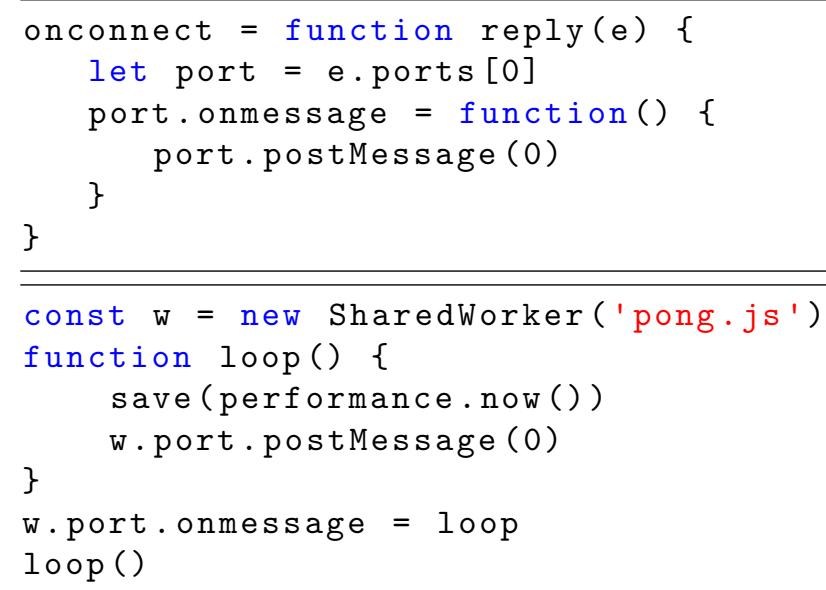

Listing 3.3: Javascript code to monitor the host's I/O thread using SharedWorkers. The first snippet is the worker's 'pong.js' file. Second snippet is the Javascript code that monitors the I/O thread by communicating with the worker.

The first snippet defines the worker's job, which consists in replying to each received message. In the second snippet, we register the worker in line 1 . In lines 2-7 we record a timestamp and recursively send messages to the worker, analogous to Listing 3.1. As a result, we measure the round-trip time from the page to the worker, which reflects the congestion in the I/O event loop. Note that one can further increase the measurement resolution by recording the time in each endpoint and merging the results. 


\subsubsection{Interferences}

There are many different sources of noise and uncertainty in the I/O thread of the host process. The most notable ones include the interleaving with the host's main thread and the messages from other renderers, but also the GPU process and browser plugins. While these interferences could potentially be exploited as side channels, the noise becomes quickly prohibitive as the loop gets crowded.

\subsubsection{The LoopScan Tool}

We implement the eavesdropping techniques described in Sections 3.1.1 and 3.1.2 in a tool called LoopScan, which enables us to explore the characteristics of the side channel caused by sharing event loops. LoopScan is based on a simple HTML page that monitors the event loops of the host and renderer processes. It relies on the D3.js framework, and provides interactive visualizations with minimap, zooming, and scrolling capabilities, which facilitates the inspection of traces. For example, Figure 3.7 is based on a screenshot from LoopScan.

LoopScan's functionality is in principle covered by the powerful Chrome Trace Event Profiling Tool (about: tracing) [40], which provides detailed flame graphs for all processes and threads. However, LoopScan has the advantage of delivering more accurate timing information about event-delay traces than the profiler, since loading a page with the Trace Event Profiling tool severely distorts the measurements. LoopScan source is publicly available at https://github.com/ cgvwzq/loopscan.

\section{$3.2 \quad$ Attacks}

In this section we systematically analyze the side channel caused by sharing event loops in three kinds of attacks: a page identification attack, an attack where we eavesdrop on user actions, and a covert channel attack. For all attacks we spy on the event loops of the renderer and the host processes, as described in Sections 3.1.1 and 3.1.2. We performed these attacks over the course of a year, always using the latest stable version of Chrome (ranging from v52-v58). The results we obtain are largely stable across the different versions.

\subsubsection{Page identification}

We describe how the event-delay trace obtained from spying on event loops can be used for identifying webpages loaded in other tabs. We begin by explaining our data selection and harvesting process and the chosen analysis methods, then we describe our experimental setup and the results we obtain. 


\subsubsection{Sample Selection}

We start with the list of Alexa Top 1000 sites, from which we remove duplicates. Here, duplicates are sites that share the subdomain but not the top-level domains (e.g., "google.br" and "google.com") and that are likely to have similar event-delay traces. From the remaining list, we randomly select 500 sites as our sample set. This reduction facilitates a rigorous exploration of the data and the parameter space.

\subsubsection{Data Harvesting}

We visit each page in the sample set 30 times for both the renderer and the host process, to record traces of event-delays during the loading phase.

The event-delay traces for the renderer process consist of 200.000 data items each. On our testing machine, the measurement resolution (i.e. the delay between two subsequent measurement events on an otherwise empty loop) lies at approximately $25 \mu \mathrm{s}$. That is, each trace captures around 5 seconds $(200.000 \cdot 25 \mu \mathrm{s}=5 \mathrm{~s})$ of the loading process of a page in the sample set.

The event-delay traces for the host process consist of 100.000 data items each. The measurement resolution lies in the range of $80-100 \mu$ s, i.e. each trace captures around $9 \mathrm{~s}$ of the loading process of a page.

We automate the harvesting procedure for the renderer process as follows:

1. Open a new tab via target $=$ window. open (URL, '_blank'); 1

2. Monitor the event loop until the trace buffer is full

3. Close the tab

4. Send the trace to the server

5. Wait 5 seconds and go to 1 with next URL

The harvesting procedure for the host process differs only in that we use the $r e l=$ "noopener" attribute in order to spawn a new renderer.

We conducted measurements on the following three machines:

1. Debian 8.6 with kernel 3.16.0-4-amd64, running on an Intel i5 @ 3.30GHz x 4 with 4 GB of RAM, and Chromium v53;

2. Debian 8.7 with kernel 3.16.0-4-amd64, running on an Intel i5-6500 @ 3.20GHz x 4 with 16 GB of RAM, and Chromium v57; and

\footnotetext{
${ }^{1}$ Note that this requires disabling Chrome's popup blocker from chrome://settings/ content.
} 
3. OSX running on a Macbook Pro 5.5 with Intel Core 2 Duo @ $2.53 \mathrm{GHz}$ with 4 GB of RAM, and Chrome v54.

We measure the timing on a Chrome instance with two tabs, one for the spy process and the other for the target page. For the renderer process, we gather data on all machines; for the host process on (2) and (3). Overall, we thus obtain five corpora of 15.000 traces each.

\subsubsection{Classification}

Event Delay Histograms. Our first approach is to cluster the observed event delays around $k$ centers, and to transform each trace into a histogram that represents the number of events that fall into each of the $k$ classes. We then use the Euclidean distance as a similarity measure on the $k$-dimensional signatures.

This approach is inspired by the notion of memprints in [33]. It appears to be suitable for classifying event-delay traces obtained from event loops because, for example, static pages with few external resources are more likely to produce long events at the beginning and stabilize soon, whereas pages with Javascript resources and animations are likely to lead to more irregular patterns and produce a larger number of long delays. Unfortunately, our experimental results were discouraging, with less than a $15 \%$ of recognition rate in small datasets.

Dynamic Time Warping. Our second approach is to maintain temporal information about the observed events. However, the exact moments at which events occur are prone to environmental noise. For example, network delays will influence the duration of network requests and therefore the arrival of events to the event loop. Instead, we focus on the relative ordering of events as a more robust feature for page identification.

This motivates the use of dynamic time warping (DTW) [41] as a similarity measure on event-delay traces. DTW is widely used for classifying time series, i.e. sequences of data points taken at successive and equally spaced points in time. DTW represents a notion of distance that considers as "close" time-dependent data of similar shape but different speed, i.e. DTW is robust to horizontal compressions and stretches. This is useful, for example, when one is willing to assign a low distance score to the time series "abc" and "abbbbc", insensitive to the prolonged duration of " $\mathrm{b}$ ". Formally, DTW compares two time series: a query, $X=\left(x_{1}, \ldots, x_{n}\right)$, and a reference, $Y=\left(y_{1}, \ldots, y_{m}\right)$. For that we use a nonnegative distance function $f\left(x_{i}, y_{i}\right)$ defined between any pair of elements $x_{i}$ and $y_{j}$. The goal of DTW is to find a matching of points in $X$ with points in $Y$, such that (1) every point is matched, (2) the relative ordering of points in each sequence is preserved (monotonicity), (3) and the cumulative distance (i.e. the sum of the values of $f$ ) over all matching points is minimized. This matching is called a warping path, and the corresponding distance is the time warping distance $d(X, Y)$. 


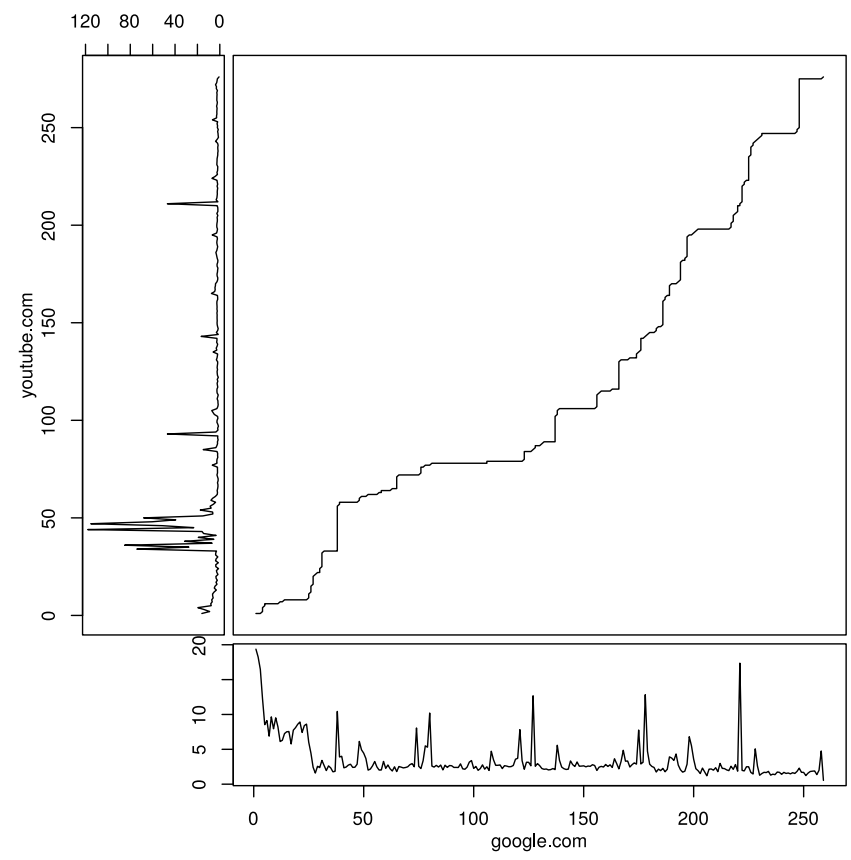

Figure 3.3: The path in the upper right square represents the optimal alignment between points in the time series corresponding to 'google.com' (horizontal axis) with points in the time series of 'youtube.com' (vertical axis).

Figure 3.3 visualizes a warping path between the time series corresponding to event-delay traces observed while loading different webpages.

\subsubsection{Speed-up Techniques}

Unfortunately, the time required for computing $d(X, Y)$ is quadratic in the length of the input sequences and does not scale up to the raw data obtained in our measurements. We rely on two kinds of speed-up techniques, one at the level of the data and the other at the level of the algorithm:

At the level of data, we reduce the dimension of our data by applying a basic sampling algorithm: We split the raw trace into groups of measurements corresponding to time intervals of duration $P$, and replace each of those groups by one representative. This representative can be computed by summing over the group, or by taking its average, maximum or minimum. The sum function generally yields the best results among different sampling functions and is the one that we use onwards. Sampling reduces the size of the traces by a factor of $P / t$, where $t$ is the average duration of an event delay. Figure 3.4 shows two plots with the raw data taken from a renderer's main thread loop, and its corresponding time series obtained after sampling.

At the algorithmic level, we use two sets of techniques for pruning the search for the optimal warping path, namely windowing and step patterns [42]. 

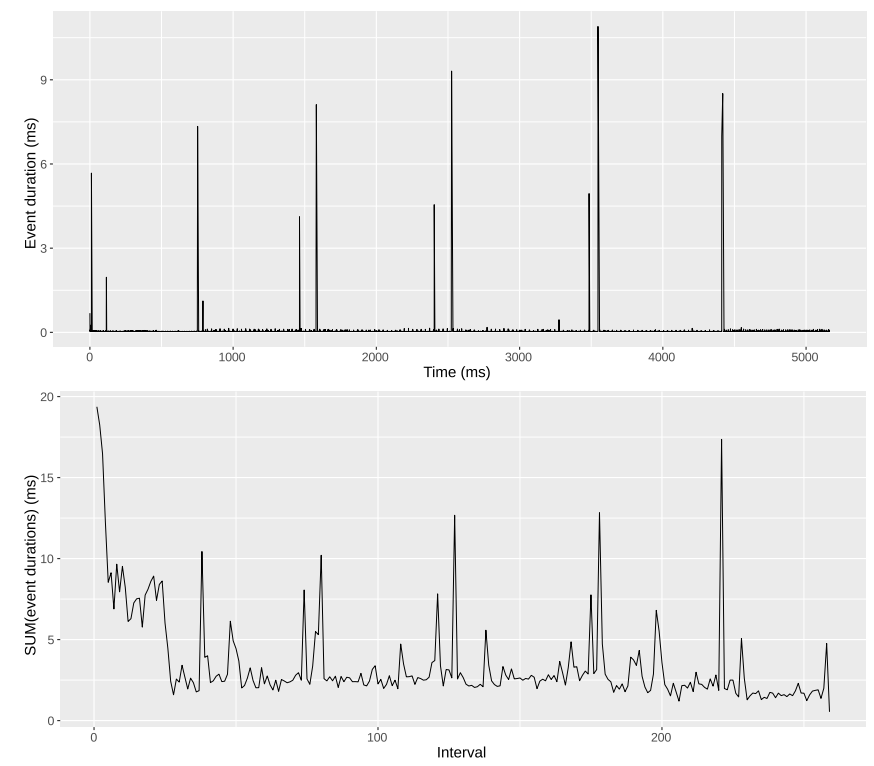

Figure 3.4: The top figure represents a raw trace of 200.000 time measurements from the renderer's main thread extracted while loading "google.com". The bottom figure displays the same data after being converted into a time series with $P=20 \mathrm{~ms}$, i.e. using only 250 data points. The difference in the height of the peaks is due to the accumulation of small events in the raw data, which are not perceptible in the top figure.

- Windowing is a heuristic that enforces a global constraint on the envelope of the warping path. It speeds up DTW but will not find optimal warping paths that lie outside of the envelope. Two well-established constraint regions are the Sakoe-Chiba band and the Itakura parallelogram, see Figure 3.5.

- Step patterns are a heuristic that puts a local constraint on the search for a warping path, in terms of restrictions on its slope. In particular, we rely on three well-known step patterns available in R. Intuitively, the symmetric1 pattern favors progress close to the diagonal, the symmetric2 pattern allows for arbitrary compressions and expansions, and the asymmetric forces each point in the reference to be used only once.

\subsubsection{Parameter tuning}

The possible configurations of the techniques presented in Section 3.2.1.4 create a large parameter space, see Table 3.1 for a summary.

We systematically identify the optimal parameter configuration for each event loop on each machine. To avoid overfitting, we divide our dataset of 30 traces (per page, loop, and machine) into 15 traces for tuning and 15 for cross-validation. For 

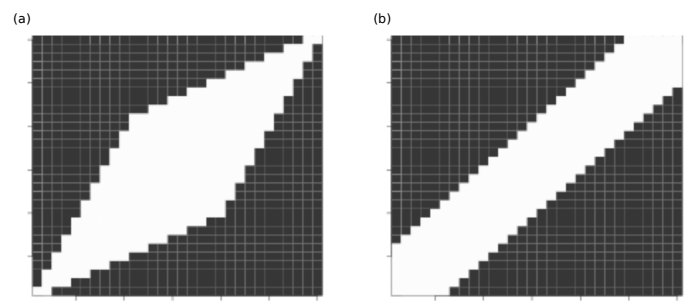

Figure 3.5: A global window constraint defines an envelope limiting the search space for optimal warping paths: (a) Itakura parallelogram, and (b) Sakoe-Chiba band.

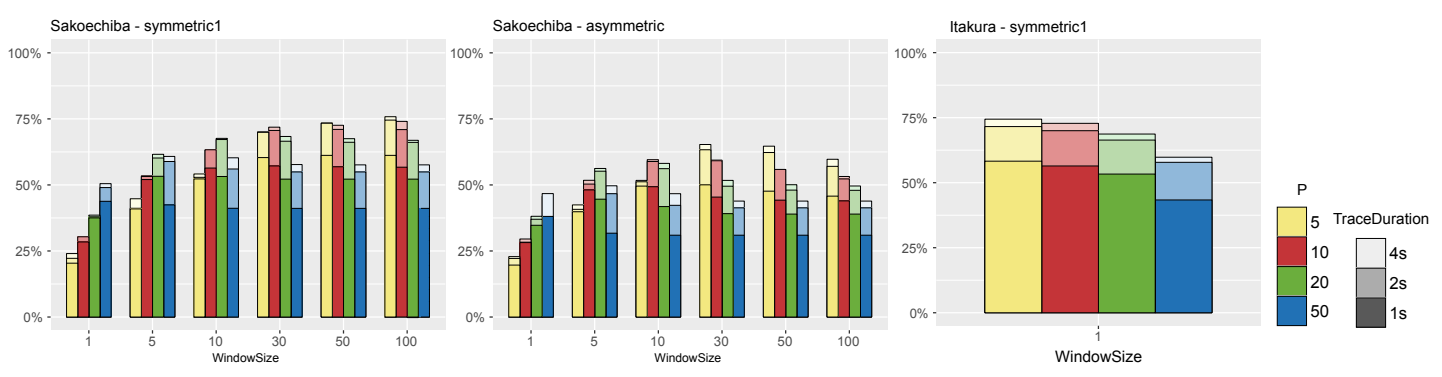

Figure 3.6: Web page identification performance after tuning with traces from the renderer on Linux machine (1). Effect of $P$, traceDuration, and windowSize, with three combinations of stepPattern and windowType.

each parameter configuration we perform a lightweight version (with 3 rounds) of the evaluation phase described in Section 3.2.1.6. Figure 3.6 visualizes an extract of the results we obtain for the renderer process of the Linux (1) machine. The tuning phase yields the following insights:

- The optimal parameters depend on the loop but appear to be stable across machines.

- Measuring the loading phase during 2 seconds is sufficient for recognition of a webpage; the gain in recognition from using longer traces is negligible.

- $P$ and windowSize are the parameters with the biggest impact on the recognition rate. However, they also have the biggest impact on the computational cost (the optimal choice being most expensive one).

- The combination of stepPattern = symmetric1 and windowType = sakoechiba generally yields the best results.

\subsubsection{Experimental Results}

We evaluate the performance of page identification through the shared event loops of host and renderer processes on each individual machine, as well as through the renderer process across two different machines. 
Chapter 3. Timing Attacks on Shared Event Loops

\begin{tabular}{|l|c|l|}
\hline Parameter & Values & Description \\
\hline traceDuration & $1000,2000,4000$ & Trace duration $(\mathrm{ms})$ \\
$P$ & $5,10,20,50$ & Sampling interval $(\mathrm{ms})$ \\
windowType & itakura, sakoechiba & Window constraint \\
windowSize & $1,5,10,30,50,100$ & Window size \\
stepPattern & symmetric1, symmetric2, & Step pattern \\
\hline
\end{tabular}

Table 3.1: List of parameters tuned for optimizing web page identification

To this end, we select the top configuration for each corpus from the tuning phase and carry out a 10-fold cross-validation. In each of the 10 rounds, we partition the validation set into a training set that contains one trace of each page, and a testing set that contains three different (out of the 14 available) traces of each page. For each of the traces in the testing set, we compute the set of $k$ closest matches in the training set according to the time warping distance.

We measure performance in terms of the $k$-match rate, which is the percentage of pages in the testing set for which the true match is within the set of $k$ closest matches. We abbreviate the 1 -match rate by recognition rate, i.e. the percentage of pages where the best match is the correct one. The result of the cross-validation is the average $k$-match rate over all 10 rounds.

Table 3.2 summarizes our experiments. We highlight the following results:

\begin{tabular}{|c|c|c|c|c|c|}
\hline & \multicolumn{4}{|c|}{$k$} \\
\hline & & 1 & 3 & 5 & 10 \\
\hline \multirow{2}{*}{ 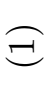 } & \multirow{2}{*}{ Renderer } & $76.7 \%$ & $86.7 \%$ & $88.8 \%$ & $91.1 \%$ \\
\hline & & \multicolumn{4}{|c|}{ sym1, sakoe, $P=5$, windowSize $=100$} \\
\hline \multirow{4}{*}{$\widehat{\mathbb{I}}$} & \multirow{4}{*}{$\begin{array}{l}\text { Renderer } \\
\text { I/O host }\end{array}$} & $58.2 \%$ & $68.6 \%$ & $71.8 \%$ & $75.1 \%$ \\
\hline & & \multicolumn{4}{|c|}{ sym1, sakoe, $P=5$, windowSize $=100$} \\
\hline & & $16.2 \%$ & $23.2 \%$ & $27.9 \%$ & $36.1 \%$ \\
\hline & & \multicolumn{4}{|c|}{ sym1, sakoe, $P=20$, windowSize $=30$} \\
\hline \multirow{4}{*}{ (2) } & \multirow{2}{*}{ Rend } & $61.8 \%$ & $74.5 \%$ & $78.4 \%$ & $83.1 \%$ \\
\hline & & \multicolumn{4}{|c|}{ sym1, sakoe, $P=5$, windowSize $=100$} \\
\hline & \multirow{2}{*}{$\mathrm{I} / \mathrm{O}$ host } & $23.48 \%$ & $32.9 \%$ & $38.1 \%$ & $46.6 \%$ \\
\hline & & \multicolumn{4}{|c|}{ sym1, sakoe, $P=20$, windowSize $=30$} \\
\hline
\end{tabular}

Table 3.2: 10-fold cross-validation results on different machines and different event loops, with the best configuration after tuning. Machines (1) and (2) refer to the Linux desktops, (3) to the OSX laptop, as described in Section 3.2.1.2.

- We can correctly identify a page by spying on the renderer from (1) in up to $76.7 \%$ of the cases, and correctly narrow down to a set of 10 candidates in up to $91.1 \%$ of the cases. 
- We can correctly identify a page though the host process from (3) in up to $23.48 \%$ of the cases, and narrow down to a set of 10 candidates in up to $46.6 \%$ of the cases.

- We stress that these recognition rates are obtained using a single trace for training.

- Recognition is easier through the renderer than through the host. This is explained by the difference in noise and measurement resolution, see Section 3.1.2.3. Furthermore, most operations on the host only block the I/O thread while signaling their start and completion, whereas the renderer is blocked during the entire execution of each Javascript task.

- We observe different recognition rates on different machines. However the homogeneity in hardware and software of Macbooks facilitate reuse of training data across machines, which may make remote page identification more feasible.

- We obtain recognition rates below $5 \%$ for recognition across machines (1) and (3). A reason for this poor performance is that events on the OSX laptop often take $2 \mathrm{x}-5 \mathrm{x}$ more time than on the Linux desktop machine. This difference is reflected in the height of the peaks (rather than in their position), which is penalized by DTW. Normalizing the measurements could improve cross-machine recognition.

The code and datasets used for tuning and cross-validation are available as an R library at https://github.com/cgvwzq/rlang-loophole.

\subsubsection{Threats to Validity}

We perform our experiments in a closed-world scenario with only 2 tabs (the spy and the victim) sharing an event loop. In real world scenarios there can be more pages concurrently running the browser, which will make detection harder. The worst case for monitoring the host process occurs when a tab performs streaming, since the loop gets completely flooded. The renderer's loop, however, is in general more robust to noise caused by other tabs in the browser.

On the other hand, our attacks do not make any use of the pages' source code or of details of Chrome's scheduling system with priority queues, the GC with periodic scavenges, or the frame rendering tasks. We believe that taking into account this information can significantly improve an adversary's eavesdropping capabilities and enable attacks even in noisy, open-world scenarios.

\subsubsection{Detecting User Behavior}

In this section we show that it is possible to detect user actions performed in a cross-origin tab or iframe, when the renderer process is shared. We first describe 
an attack recovering the inter-keystroke timing information against Google's OAuth login forms, which provides higher precision than existing network-based attacks [37].

\subsubsection{Inter-keystroke Timing Attack on Google's OAuth login form}

Many web applications use the OAuth protocol for user authentication. OAuth allows users to login using their identity with trusted providers, such as Google, Facebook, Twitter, or Github. On the browser, this process is commonly implemented as follows:

1. A web application $A$ pops up the login form of a trusted provider $T$;

2. User $V$ types their (name and) password and submits the form to $T$;

3. $T$ generates an authorization token.

Because the window of the login form shares the event loop with the opener's renderer, a malicious $A$ can eavesdrop on the keystroke events issued by the login form.

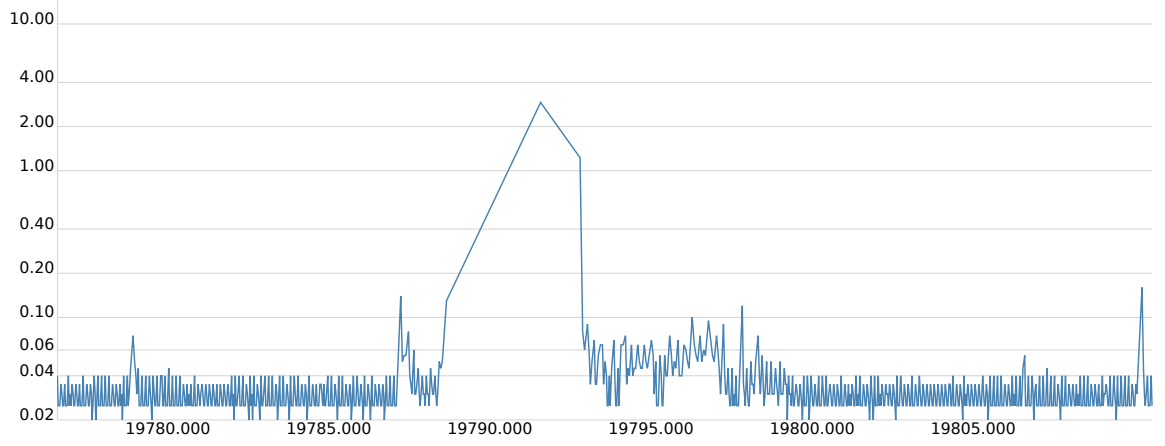

Figure 3.7: Delay pattern generated by a keystroke in the Google OAuth login form, measured across origins on Chrome Canary v61 on OSX. The two consecutive delays of approx. 2ms each, correspond to keydown and keypress event listeners.

Figure 3.7 depicts the event-delay trace of a keystroke as seen by an eavesdropper on the renderer's event loop. The trace contains two characteristic consecutive delays, caused by the keydown and keypress event listeners. We use this observation to identify keystrokes, by scanning the event-delay trace for pairs of consecutive delays that are within a pre-defined range, forgoing any training or offline work. Listing 3.4 contains the script that performs this operation. We define $0.4 \mathrm{~ms}$ as a lower bound, and $3.0 \mathrm{~ms}$ as an upper bound for the range. We chose this threshold before gathering the data, by manual inspection of a few keystroke events. Note that this calibration could be done automatically, based on the victim's interactions with a page controlled by an attacker. 


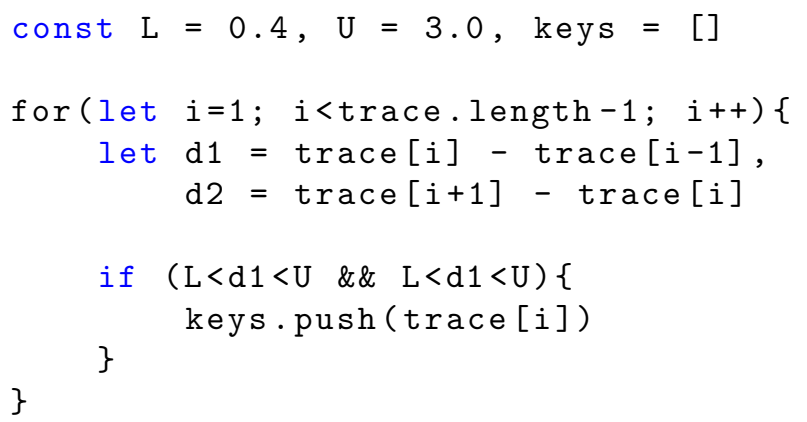

Listing 3.4: Pseudo-Javascript code to detect keystrokes in a trace of timestamps gathered by the code in Listing 3.1. We classify a timestamp as a keystroke if the differences to the previous and subsequent timestamps ( $d 1$ and $d 2)$ are both in a predefined range.

\subsubsection{Experimental Evaluation}

To evaluate the effectiveness of this attack, we have implemented a malicious application $A$ that extracts the inter-keystroke timing information from a user $V$ logging-in via Google's OAuth. The focus of our evaluation is to determine the accuracy with which keystroke timings can be measured through the event loop. A full keystroke recovery attack is out of scope of this work; for this refer to [37].

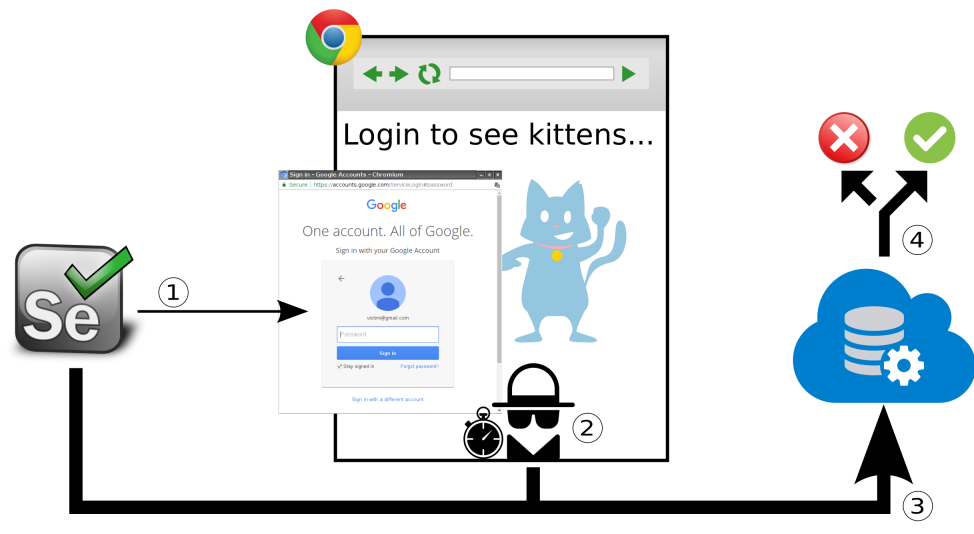

Figure 3.8: Experimental setup for evaluating effectiveness of automatic, crossrenderer keystroke detection.

We simulate an inter-keystroke timing attack in 4 steps, which are described below and illustrated in Figure 3.8.

1. A Selenium ${ }^{2}$ script acting as $V$ navigates to $A$, clicks on the login button

\footnotetext{
${ }^{2}$ Selenium (http://www.seleniumhq.org/) is a cross-platform testing framework for web applications that provides capabilities for programmatically navigating to web pages and producing user input.
} 
(which pops up Google's OAuth login form), types a password, and submits the form.

2. Meanwhile, the attacker $A$ monitors the main thread's event loop using the attack described in Section 3.2.2.1.

3. $V$ and $A$ send to the server the timestamps of the real and the detected keystrokes, respectively.

4. We compute the accuracy of the detected keystrokes, where we take the timestamps of the real keystrokes as ground truth. Matching the timestamps requires taking into account the delay ( $6-12 \mathrm{~ms}$ on our machine) between Selenium triggering an event, and Chrome receiving it.

We use as inter-keystroke timings random delays uniformly drawn from $100-$ $300 \mathrm{~ms}$. This choice is inspired by [34], who report on an average inter-keystroke delay of $208 \mathrm{~ms}$. Using random delays is sufficient for evaluating the accuracy of eavesdropping on keystrokes, but it obviously does not reveal any information about the password besides its length.

\subsubsection{Experimental Results}

We perform experiments with 10.000 passwords extracted from the RockYou dataset, where we obtain the following results:

- In $91.5 \%$ of the cases, our attack correctly identifies the length of a password. ${ }^{3}$ In $2.2 \%$ of the cases, the attack misses one or more characters, and in $6.3 \%$ of the cases it reports spurious characters.

- For the passwords whose length was correctly identified, the average time difference between a true keystroke and a detected keystroke event is $6.3 \mathrm{~ms}$, which we attribute mostly to the influence of Selenium. This influence cancels out when we compute the average difference between a true interkeystroke delay and a detected inter-keystroke delay, which amounts to $1.4 \mathrm{~ms}$. The noise of these measurements is low: We observe a standard deviation of $6.1 \mathrm{~ms}$, whereas the authors of [34] report on $48.1 \mathrm{~ms}$ for their network based measurements.

Overall, our results demonstrate that shared event loops in Chrome enable much more precise recovery of keystroke timings than network-based attacks. Moreover, this scenario facilitates to identify the time when keystroke events enter the loop (from popping-up to form submission), which is considered to be a major obstacle for inter-keystroke timing attacks on network traffic [34].

\footnotetext{
${ }^{3}$ We configured Selenium to atomically inject characters that would require multiple keys to be pressed.
} 
Keystroke timing attacks based on monitoring procfs [35] or CPU caches [36] can extract more fine-grained information about keystrokes, such as containment in a specific subsets of keys. However, they require filesystem access or are more susceptible to noise, due to the resource being shared among all processes in the system. In contrast, our attack enables targeted eavesdropping without specific privileges.

\subsubsection{Open Challenges for Recognizing User Events}

We conclude by discussing two open challenges for recognizing user events, namely the detection of user events beyond keystrokes and the detection of events in the browser's host process.

Detecting User Events beyond Keystrokes A continuous mouse movement results in a sequence of events, each of which carrying information about the coordinates of the cursor's trajectory. These events are issued with an interevent delay of $8 \mathrm{~ms}$, and the (empty) event listener operation blocks the loop for approx $0.1 \mathrm{~ms}$. The particular frequency and duration of these events makes mouse movements (or similar actions, like scrolling) easy to spot with LoopScan, as seen in Figure 3.9.

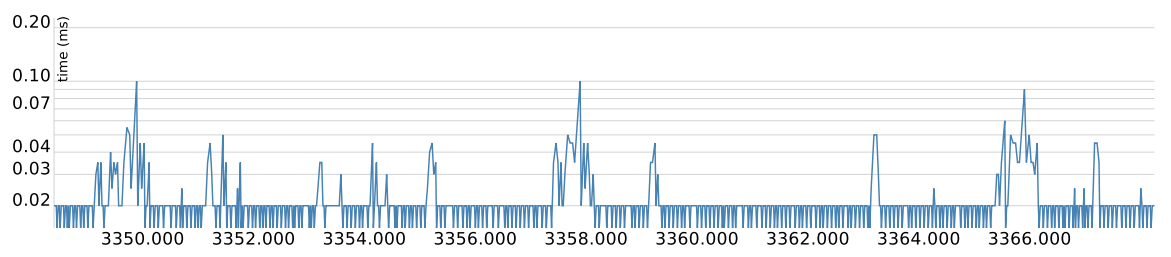

Figure 3.9: Mouse movement captured by LoopScan tool. The graph shows 3 delays of $0.1 \mathrm{~ms}$ duration (at $t$ equals 3350,3358 and 3366), with an inter-event delay of $8 \mathrm{~ms}$.

Likewise, mouse click events, corresponding to "up" or "down", can be identified using LoopScan. Their shape depends on the specific event listener of the spied web page and the HTML element being clicked. Figure 3.10 illustrates the effect of a mouse down event in the renderer's event loop. The empty event handling operation is reflected by the two consecutive delays of around $0.5 \mathrm{~ms}$. We expect that events with specific listeners are more easily detectable than events without registered event listeners, that is, user actions that do not trigger Javascript execution. However, we can use the context in which the event occurs to reduce the search space. For instance, most mouse clicks only appear between two sequences of mouse movement events.

We are currently investigating techniques that enable the automatic identification of such patterns in event-delay streams. A promising starting point for this are existing on-line variants of dynamic time-warping [43]. 


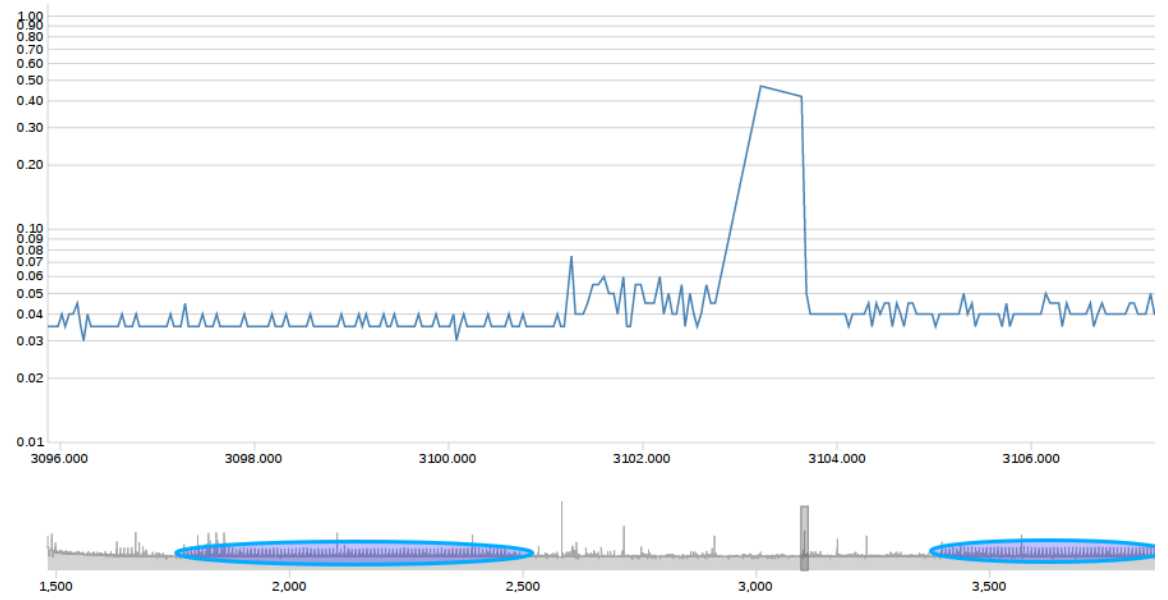

Figure 3.10: Mouse down event captured by LoopScan tool. The bottom part shows the minimap (the highlighted intervals correspond to mouse movement); the top part shows a zoomed selection. Horizontal axis depicts elapsed real time; vertical axis depicts event delays, all in milliseconds.

Detecting User Events in the Host Process Our discussion so far has centered on detecting user events in the event loop of the renderer process. However, all user events originate in the main thread of the host process and are sent towards a specific renderer through the event loop of the host's I/O thread. Hence, any user action can in principle be detected by spying on the host.

Unfortunately, our current methods are not precise enough for this task, since the host's I/O thread is more noisy than the renderer's main thread and the effect of a user action on the host process is limited to a short signaling message, whereas the renderer's main thread is affected by the execution of the corresponding Javascript event listener.

\subsubsection{Covert Channel}

In this section we show how shared event loops in Chrome can be abused for implementing covert channels, i.e. channels for illicit communication across origins. We first consider the case of cross-origin pages sharing the event loop of a renderer's main thread before we turn to the case of cross-origin pages sharing the event loop of the host processes' I/O thread.

\subsubsection{Renderer Process}

We implement a communication channel to transmit messages from a sender page $S$ to a cross-origin receiver page $R$ running in the same renderer process.

For this, we use a simple, unidirectional transmission scheme without error correction. Specifically, we encode each bit using a time interval of fixed duration 
$t_{b}$. The optimal configuration of $t_{b}$ depends on the system. In our experiments we tried different values, with $t_{b}=5 \mathrm{~ms}$ giving good results on different platforms: Chromium 52.0 on Debian 64-bit and Chrome 53 on OSX.

In each of those intervals we do the following:

- the sender $S$ idles for transmitting a 0 ; it executes a blocking task of duration $\hat{t}<t_{b}$ for transmitting a 1 .

- the receiver $R$ monitors the event loop of the renderer's main thread using the techniques described in Section 3.1.1; it decodes a 0 if the length of the observed tasks is below a threshold (related to $\hat{t}$ ), and a 1 otherwise.

Transmission starts with $S$ sending a 1, which is used by the agents to synchronize their clocks and start counting time intervals. Transmission ends with $S$ sending a null byte. With this basic scheme we achieve rates of $200 \mathrm{bit} / \mathrm{s}$. These numbers can likely be significantly improved by using more sophisticated coding schemes with error correction mechanisms; here, we are only interested in the proof-ofconcept.

We note that there are a number of alternative covert channels for transmitting information between pages running in the same renderer [44], e.g., using window.name, location.hash, history.length, the scrollbar's position or frames. length. What distinguishes the event-loop based channel is that it does not require the sender and receiver to be connected, i.e. they do not need to hold references to each other in order to communicate.

\subsubsection{Host Process}

We also implement a communication channel to transmit messages between two cooperative renderer processes sharing the host process. Transmission is unidirectional from sender $S$ to receiver $R$. Figure 3.11 visualizes how this channel can be used, even if one of the parties browses in Incognito mode.

As before, we encode each bit using a time intervals of fixed duration $t_{b}$. During each intervals we do the following:

- the sender $S$ idles for transmitting a 0 ; it posts $N$ fetch requests into the I/O thread's queue for sending a 1.

- the receiver $R$ monitors the event loop of the I/O thread of the host process using the techniques described in Section 3.1.2. It decodes a 0 if the number of observed events during time interval $t_{b}$ is below a threshold, and 1 otherwise.

The optimal values of $N$ and $t_{b}$ highly depend on the machine. In our experiments we achieve good results, working on different systems, with a $t_{b}=200 \mathrm{~ms}$ and $N=350$, which give us a $5 \mathrm{bit} / \mathrm{s}$ transmission rate. This rate is significantly 


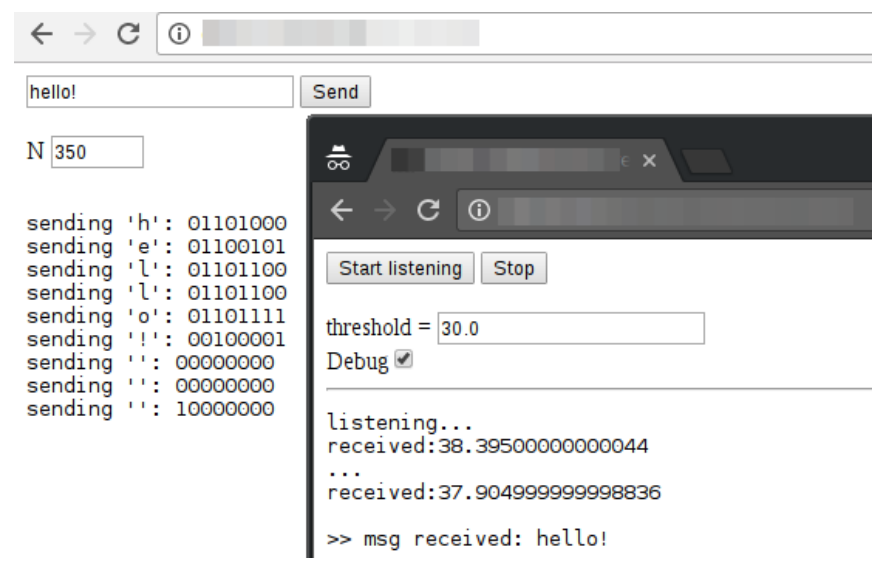

Figure 3.11: Covert channel through the I/O event loop of the Chrome's host process. Tabs in different renderer processes (one of them navigating in Incognito mode) communicate.

lower than for communication using the renderer event loop, which is explained by the difference in noise and monitoring resolution of both channels, as discussed in Section 3.1.2.3.

The threat scenario of this covert channel is more relevant than the previous one for the renderer loop. For example it could be used for exfiltrating information from an attacked domain (on a tab executing malicious Javascript). Using Workers (which are background threads that run independently of the user interface) we can transfer information across origins, without affecting the user experience and without generating network traffic.

\subsection{Discussion}

We have shown how sharing event loops leads to timing side-channels and presented different attacks on Chrome. We communicated our findings to the Chromium security team, who decided not to take action for the time being. Nevertheless, our results point to fundamental security issues in the event-driven architecture of browsers that eventually need to be addressed in a fundamental manner. Below, we discuss how other platforms are affected and present possible countermeasures.

\subsubsection{Beyond Chrome}

We focus on Chrome in our analysis because it is the most widely used browser, and because it was the first one to implement a multi-process architecture. However, there are good reasons to expect similar side channels in other browsers, as they all follow the same event-driven paradigm and rely on similar architectures. 
For instance, recent Firefox versions with multi-process support ${ }^{4}$ also rely on a privileged browser process and multiple content processes that, unlike renderers in Chrome, act as a pool of threads for each different origin (each with its own message queue). Despite this difference, tests with LoopScan on Firefox version 55 show that congestion on both event loops is observable across origins and tabs.

Specifically, we applied the monitoring technique for the renderers described in Section 3.1.1.2 on a micro-benchmark with a set of 30 pages with 15 traces each. We achieved a recognition rate of $49 \%$, which is below the recognition rate achieved on Chrome for a set of 500 pages. A fair comparison between both architectures will require a better understanding of Firefox's policy for mapping sites to threads and events to loops.

\subsubsection{Countermeasures}

The attacks presented rely on two capabilities of the adversary: (1) the ability to post tasks into the loop's queue with high frequency, and (2) the ability to accurately measure the corresponding time differences.

Rate Limiting. An obvious approach to counter (1) is to impose a limit on the rate at which tasks can be posted into an event loop. Unfortunately, rate limiting implies penalties on performance, which is especially problematic for asynchronous code.

At the level of the renderer, one possibility is to rely on an accumulate and serve policy [32]. With this policy, the event loop accumulates all the incoming jobs in a buffer for a period $T$, and then process and serves all the accumulated jobs from party $A$, followed by all the jobs from $V$. This has the advantage of limiting the amount of information leaked while retaining high amortized throughput.

At the level of the host process, where resource fetching is one of the main performance concerns, setting any bound on the processing rate is not acceptable. Here, it seems more reasonable to monitor the IPC activity of all renderers and penalize or flag those who exhibit a bad or anomalous behavior, e.g., along the lines of $[45]$.

Reduce Clock Resolution. An obvious approach to counter (2) is to limit the resolution of available clocks. This has already been applied by browser vendors for mitigating other kinds timing channels, but these efforts are unlikely to succeed, as shown in [15]: Modern browsers have a considerable number of methods to measure time without any explicit clock. For instance, some recent exploits [46] use high-resolution timers build on top of SharedArrayBuffers. The current res-

\footnotetext{
${ }^{4}$ Firefox's Electrolysis (or e10s) project
} 
olution of performance.now is limited to $5 \mu \mathrm{s}$, which makes microarchitectural timing attacks difficult, but does not preclude the detection of Javascript events.

Full Isolation. As discussed in Section 2.1.2, Chrome's multi-process architecture tries to use a different renderer for different origins, except for some corner cases. The "Site Isolation Project" is an ongoing effort to ensure a complete process-per-site-instance policy, that means: providing cross-process navigations, cross-process Javascript interactions and out-of-process iframes. All this without inducing too much overhead.

One open question is how to handle the system's process limit, namely which sites should have isolation preference, or which heuristic for process reuse should be used. A recent proposal, "IsolateMe" [47], puts the developers in charge of requesting to be isolated from other web content (even if it does not provide a firm guarantee).

CPU Throttling. Chrome v55 introduces an API that allows to limit how much CPU a background page is allowed to use, and to throttle tasks when they exceed this limit. This affects background tabs trying to spy on the renderer's main thread, but still allows spying on (and from) any iframe and popup, as well as on the I/O thread of the host process through shared Workers. Moreover, background tabs with audio activity are not affected, as they are always marked as foreground. Since Chrome v57 pages (or tabs) are only subjected to throttling after 10 seconds in the background, which is too long to prevent the attacks presented.

\subsection{Related Work}

Timing attacks on web browsers date back to Felten and Schneider [48], who use the browser cache to obtain information about a user's browsing history.

More recently, so-called cross-site timing attacks [49, 50] have exploited the fact that the browser attaches cookies to all requests, even when they are performed across origins. The presence or absence of these cookies can be determined by timing measurements, which reveals information about the user's state on arbitrary sites. A special case are cross-site search attacks [51], which circumvent the same-origin policy to extract sensitive information, by measuring the time it takes for the browser to receive responses to search queries.

Other classes of browser-based timing attacks exploit timing differences in rendering operations $[52,53,54]$, or simply use the browser as an entry point for Javascript that exploits timing channels of underlying hardware, for example caches [55, 46], DRAM buffers [56], or CPU contention [57].

Of those approaches, [57] is related to our work in that it identifies web pages across browser tabs, based on timing of Javascript and a classifier using 
dynamic time warping. However, because the attack relies on CPU contention as a channel, it requires putting heavy load on all cores for monitoring. In contrast, our attack exploits the browser's event loop as a channel, which can be monitored by enqueing one event at a time. This makes our attack stealthy and more independent of the execution platform.

To the best of our knowledge, we are first to mount side-channel attacks that exploit the event-driven architecture of web browsers. Our work is inspired by a proof-of-concept attack [58] that steals a secret from a cross-origin web application by using the single-threadedness of Javascript. We identify Chrome's event-driven architecture as the root cause of this attack, and we show how this observation generalizes, in three different attacks against two different event loops in Chrome.

Finally, a central difference between classical site fingerprinting [59, 60, 61, 62] approaches and our page identification attack is the adversary model: First, our adversary only requires its page to be opened in the victim's browser. Second, instead of traffic patterns in the victim's network, our adversary observes only time delays in the event queues of the victim's browser. We believe that our preliminary results, with up to $76 \%$ of recognition rate using one single sample for training in a closed-world with 500 pages, can be significantly improved by developing domain-specific classification techniques. 


\section{Finding Eviction Sets}

Attacks against the micro-architecture of modern CPUs have rapidly evolved from an academic stunt to a powerful tool in the hand of real-world adversaries. Prominent examples of attacks include side-channel attacks against shared CPU caches [63], fault injection attacks against DRAM [6], and covert channel attacks that leak information from speculative executions [13].

A key requirement for many of the documented attacks is that the adversary be able to bring specific cache sets into a controlled state. For example, flush+reload [63] attacks use special instructions to invalidate targeted cache content (like clflush on x86), for which they require privileged execution and shared memory space. Another class of attacks, called prime+probe, evicts cache content by replacing it with new content and can be performed without privileges from user space or from a sandbox.

The primitive used for replacing cache content is called an eviction set. Technically, an eviction set is a collection of (virtual) addresses that contains at least as many elements that map to a specific cache set as the cache has ways. The intuition is that, when accessed, an eviction set clears all previous content from the cache set. Eviction sets enable an adversary to (1) bring specific cache sets into a controlled state; and to (2) probe whether this state has been modified by the victim, by measuring latency of accesses to the eviction set.

Accessing a large enough set of virtual addresses is sufficient for evicting any content from the cache. However, such large eviction sets increase the time required for evicting and probing, and they introduce noise due to the unnecessary memory accesses. For targeted and stealthy eviction of cache content one hence seeks to identify eviction sets of minimal size, which is fundamental, for example, for

- fine-grained monitoring of memory usage by a concurrent process in timing attacks against last-level caches [64, 65];

- enforcing that memory accesses hit DRAM instead of the cache with high 
enough frequency to flip bits in rowhammer attacks [66];and

- increasing the number of instructions that are speculatively executed by ensuring that branch guards are not cached [13].

Computing minimal eviction sets is recognized as a challenging problem, equivalent to learning the mapping from virtual addresses to cache sets [64]. The difficulty of the problem is governed by the amount of control the adversary has over the bits of physical addresses. For example, on bare metal, the adversary completely controls the mapping to cache sets; on huge pages, it controls the mapping to cache sets within each cache slice, but not the mapping to slices; on regular $4 \mathrm{~KB}$ pages, it only partially controls the mapping to sets within each slice; and on sandboxed or hardened environments it may not have any control over the mapping at all $[67,65]$.

Several approaches in the literature discuss algorithms for finding minimal eviction sets, see Section 5.9 for an overview. These algorithms rely on a twostep approach in which one first collects a large enough set of addresses that is an eviction set, and then successively reduces this set to its minimal core. Unfortunately, these algorithms are usually only considered as a means to another end, such as devising a novel attack. As a consequence, they still lack an in-depth analysis in terms of complexity, real-time performance, correctness, and scope, which hinders progress in research on attacks and on principled countermeasures at the same time.

Our approach In this work we perform the first systematic study of finding minimal eviction sets as an algorithmic problem. In our study we proceed as follows:

- We give the first formalization and analysis of the problem of finding eviction sets. We study different variants of the problem, corresponding to different goals, for example, "evicting a specific cache set", and "evicting an arbitrary cache set". For these goals, we express the probability that a set of virtual addresses is an eviction set as a function of its size. The function exhibits that a small set of virtual addresses is unlikely to be an eviction set, but that the likelihood grows fast with the set size. This analysis justifies the two-step approach taken in the literature for computing minimal eviction sets, and it exhibits favorable set sizes to start the reduction.

- We design novel algorithms for finding minimal eviction sets. The basis of our algorithms are tests from the literature [64] that use the cache sidechannel as an oracle to determine whether a given set of virtual addresses is an eviction set. The key observation underlying our algorithms is that these tests can be seen as so-called threshold group tests [68]. This observation allows us to leverage ideas from the group testing literature for computing 
minimal eviction sets. We show that the resulting algorithm reduces an eviction set of size $n$ to its minimal core using only $\mathcal{O}(n)$ memory accesses, which improves over the current $\mathcal{O}\left(n^{2}\right)$ state-of-the-art [69].

- We perform a rigorous reliability analysis of our algorithms on Intel's Haswell and Skylake microarchitectures. In our analysis, we identify ways to isolate the influence of TLBs and cache replacement policies. This allows us to exhibit conditions under which our algorithms are almost perfectly reliable, as well as conditions under which their reliability degrades.

- We carry out a performance analysis of our algorithms on Intel Skylake. Our analysis shows that the execution time of our algorithms indeed grows only linearly in practice, which leads to significant speed-up compared to the existing quadratic algorithms. While previous approaches rely on assumptions about the number of controlled physical bits (provided by huge and regular pages), our algorithms enable, for first time, computing eviction sets in scenarios without any control of the mapping from virtual addresses to cache sets, as in $[67,65]$.

Summary of contributions Our contributions are both theoretical and practical. On the theoretical side, we formalize the problem of finding minimal eviction sets and devise novel algorithms that improve the state-of-the-art from quadratic to linear. On the practical side, we perform a rigorous empirical analysis that exhibits the conditions under which our algorithms succeed or fail. Overall, our insights provide a basis for principled countermeasures against, or paths for further improving the robustness of, algorithms for finding eviction sets.

We also include a tool for evaluating, on different platforms, all tests and algorithms presented:

https://github.com/cgvwzq/evsets

\subsection{Eviction Sets}

In this section we give the first formalization of eviction sets, and present tests that enable determining whether a given set of addresses is an eviction set. We then express the probability that a set of random addresses forms an eviction set as a function of its size. The development of this section forms the basis for the algorithms we develop, and for their evaluation.

\subsubsection{Defining Eviction Sets}

We say that two virtual addresses $x$ and $y$ are congruent, denoted by $x \simeq y$, if they map to the same cache set. This is the case if and only if the set index bits 
set $(\cdot)$ and slice bits slice $(\cdot)$ of their respective physical addresses $p t(x)$ and $p t(y)$ coincide. That is, $x \simeq y$ if and only if:

$$
\operatorname{set}(p t(x))=\operatorname{set}(p t(y)) \wedge \operatorname{slice}(p t(x))=\operatorname{slice}(p t(y))
$$

Congruence is an equivalence relation. The equivalence class $[x]$ of $x$ w.r.t. $\simeq$ is the set of virtual addresses that maps to the same cache set as $x$. We say that addresses are partially congruent if they satisfy the first term of Equation (4.1), i.e., they coincide on the set index bits but not necessarily on the slice bits.

We now give definitions of eviction sets, where we distinguish between two goals: In the first, we seek to evict a specific address from the cache. This is relevant, for example, to perform precise flushing in rowhammer attacks. In the second, we seek to evict the content of an arbitrary cache set. This is relevant, for example, for high bandwidth covert channels, where one seeks to control a large number of cache sets, but does not care about which ones.

Definition 1. We say that a set of virtual addresses $S$ is

- an eviction set for $x$ if $x \notin S$ and at least $a$ addresses in $S$ map to the same cache set as $x$ :

$$
|[x] \cap S| \geq a
$$

- an eviction set (for an arbitrary address) if there exists $x \in S$ such that $S \backslash\{x\}$ is an eviction set for $x$ :

$$
\exists x:|[x] \cap S| \geq a+1
$$

The intuition behind Definition 1 is that sequentially accessing all elements of an eviction set for $x$ will ensure that $x$ is not cached afterwards. Likewise, sequentially accessing $a+1$ congruent elements will guarantee that at least one of them is being evicted.

For this intuition to hold, the cache replacement policy needs to satisfy a condition, namely that a sequence of $a$ misses to a cache set evicts all previous content. This condition is satisfied, for example, by all permutation-based policies [70], which includes LRU, FIFO, and PLRU. However, the condition is only partially satisfied by modern (i.e. post Sandy Bridge) Intel CPUs. See Section 4.4 for a more detailed discussion.

\subsubsection{Testing Eviction Sets}

Identifying eviction sets based on Definition 1 involves checking whether (4.1) holds. This requires access to bits of the physical addresses and cannot be performed by user programs. In this section we present tests that rely on a timing side-channel to determine whether a set of virtual addresses is an eviction set. 


$$
a_{v} a_{0} a_{1} \ldots a_{n-1} a_{v}^{3}
$$

Test 4.1: Eviction test for a specific address $a_{v}$ : (1) Access $a_{v}$. (2) Access $S=$ $\left\{a_{0}, \ldots, a_{n-1}\right\}$. (3) Access $a_{v}$. If the time for (3) is larger than a threshold, then $S$ is an eviction set for $a_{v}$.

$$
a_{0} a_{1} \ldots a_{n-1} a_{0} a_{1} \ldots a_{n-1}
$$

Test 4.2: Eviction test for an arbitrary address: (1) Access $S=\left\{a_{0}, \ldots, a_{n-1}\right\}$. (2) Access $S$ again. If the overall time for (2) is above a threshold, $S$ is an eviction set.

- Test 4.1 from $[64,65]$ enables user programs to check whether $S$ is an eviction set for a specific virtual address $a_{v}$. The test relies on the assumption that a program can determine whether $a_{v}$ is cached or not. In practice, this is possible whenever a program has access to a clock that enables it to distinguish between a cache hit or a miss.

Test 4.1 can also be used as a basis for testing whether a set $S$ is an eviction set for an arbitrary address, by running $\operatorname{TEST}\left(S \backslash\left\{a_{i}\right\}, a_{i}\right)$, for all $a_{i} \in S$, and reporting any positive outcome. However, the number of memory accesses required for this is quadratic in the size of $S$.

- Test 4.2 is a more efficient solution that has been informally discussed in [65]. The idea is to iterate over all the elements of $S$ twice, and measure the overall time of the second iteration. The first iteration ensures that all elements are cached. If the time for the second iteration is above a certain threshold, one of the elements has been evicted from the cache, showing that $S$ is an eviction set. The downside of Test 4.2 is that it is susceptible to noise, in the sense that any delay introduced during the second iteration will result in a positive answer.

- We propose Test 4.3 as a variant of Test 4.2, drawing inspiration from Horn's proof-of-concept implementation of Spectre [71]. By measuring the individual time of each access instead of the overall access time one can (1) reduce the time window in which spurious events can pollute the measurements, and (2) count the exact number of cache misses in the second iteration. While this improves robustness to noise, it also comes with a cost in terms of the number of executed instructions.

\subsubsection{The Distribution of Eviction Sets}

In this section we analyze the distribution of eviction sets. More specifically, we compute the probability that a suitably chosen set of random virtual addresses 


$$
a_{0} a_{1} \ldots a_{n-1} a_{0}^{3} a_{1}^{3} \ldots a_{n-1}
$$

Test 4.3: Robust eviction test for an arbitrary address: (1) Access $S=$ $\left\{a_{0}, \ldots, a_{n-1}\right\}$. (2) Access $S$ again, measuring access time of each element. If the access times of more than $a$ elements in (2) is above a threshold, $S$ is an eviction set.

forms an eviction set, for different degrees of adversary control.

\section{Choosing Candidate Sets}

For explaining what "suitably chosen" means, we need to distinguish between the $\gamma$ set index bits of the physical addresses that can be controlled from user space, and the $c-\gamma$ bits that cannot. The value of $\gamma$ depends, for example, on whether we are considering huge or small pages.

Controlling set index bits from user space is possible because the virtual-tophysical translation $p t$ acts as the identity on the page offset, see Section 2.2.2. When trying to find a minimal eviction set, one only needs to consider virtual addresses that coincide on those $\gamma$ bits.

The challenge is to find collisions on the $c-\gamma$ set index bits that cannot be controlled from user space (the page color bits in Figure 2.2), as well as on the unknown $s$ slice bits. In this section, we assume that the virtual-tophysical translation $p t$ acts as a random function on those bits. This assumption corresponds to the worst case from an adversary's point of view; in reality, more detailed knowledge about the translation can reduce the effort for finding eviction sets [72].

Whenever we speak about "choosing a random set of virtual addresses" of a given size, we hence refer to choosing random virtual addresses that coincide on all $\gamma$ set index bits under control. We now determine the probability of such a set to be an eviction set.

\section{Probability of Colliding Virtual Addresses}

We first compute the probability that two virtual addresses $y$ and $x$ that coincide on the $\gamma$ user-controlled set index bits are actually congruent. We call this event a collision and denote it by $C$. As $p t$ acts as a random function on the remaining $c-\gamma$ set index bits and $s$ slice bits, we have:

$$
P(C)=2^{\gamma-c-s}
$$

The following example illustrates how removing adversary control increases the difficulty of finding collisions on common cache configurations.

Example 1. Consider the cache from Figure 2.2 with 8 slices (i.e. $s=3$ ) of 1024 cache sets each (i.e. $c=10$ ). 
- With huge pages (i.e. $p=21$ ), the attacker controls all of the set index bits, i.e. $\gamma=c$, hence the probability of a collision $P(C)=2^{-3}$ is given by the number of slices.

- With pages of $4 \mathrm{~KB}$ (i.e. $p=12$ ), the number of bits under control is $\gamma=p-\ell=6$, hence the probability of finding a collision is $P(C)=2^{6-10-3}=2^{-7}$.

- The limit case (i.e. $p=\ell=6$ ) corresponds to an adversary that has no control whatsoever over the mapping of virtual addresses to set index bits and slice bits - besides the fact that a virtual address always maps to the same physical address. This case corresponds to adding a permutation layer to all adversarycontrolled bits (see, e.g. [67]) and is a candidate for a countermeasure that makes finding eviction sets intractable. For this case we obtain $P(C)=2^{-10-3}=2^{-13}$.

\section{Probability of a Set to be an Eviction Set for $x$}

We analyze the probability of a set of virtual addresses $S$ to be an eviction set for a given address $x$. This probability can be expressed in terms of a binomially distributed random variable $X \sim B(N, p)$ with parameters $N=|S|$ and $p=$ $P(C)$. With such an $X$, the probability of finding $k$ collisions, i.e., $|S \cap[x]|=k$, is given by:

$$
P(X=k)=\left(\begin{array}{c}
N \\
k
\end{array}\right) p^{k}(1-p)^{N-k}
$$

According to Definition 1, $S$ is an eviction set if it contains at least $a$ addresses that are congruent with $x$, see (1). The probability of this happening is given by:

$$
\begin{aligned}
P(|S \cap[x]| \geq a) & =1-P(X<a) \\
& =1-\sum_{k=0}^{a-1}\left(\begin{array}{l}
N \\
k
\end{array}\right) p^{k}(1-p)^{N-k}
\end{aligned}
$$

Figure 4.4 depicts the distribution of sets to be an eviction set for $x$, based on the cache from Figure 2.2.

\section{Probability of a Set to be an Eviction Set for an arbitrary address}

We analyze the probability that a set $S$ contains at least $a+1$ addresses that map to the same cache set. To this end, we view the problem as a cell occupancy problem.

Namely, we consider $B=2^{s+c-\gamma}$ possible cache sets (or bins) with $N=|S|$ addresses (or balls) that are uniformly distributed, and ask for the probability of filling at least one set (or bin) with more than $a$ addresses (or balls).

We model this probability using random variables $N_{1}, \ldots, N_{B}$, where $N_{i}$ represent the number of addresses mapping to the $i$-th cache set, with the constraint that $N=N_{1}+\cdots+N_{B}$. With this, the probability of having at least one set 
with more than $a$ addresses can be reduced to the complementary event of all $N_{i}$ being less or equal than $a$ :

$$
P\left(\exists i \mid N_{i}>a\right)=1-P\left(N_{1} \leq a, \ldots, N_{B} \leq a\right)
$$

The right-hand side is a cumulative multinomial distribution, whose exact combinatorial analysis is expensive for large values of $N$ and becomes unpractical for our purpose. Instead, we rely on a well-known approximation based on Poisson distributions [73] for calculating the probabilities.

Figure 4.4 depicts the distribution of sets to be an eviction set for an arbitrary address, based on the cache from Figure 2.2. We observe that the probability of the multinomial grows faster with the set size than the binomial distribution. This shows that a set is more likely an eviction set for an arbitrary address than it is for a specific address.

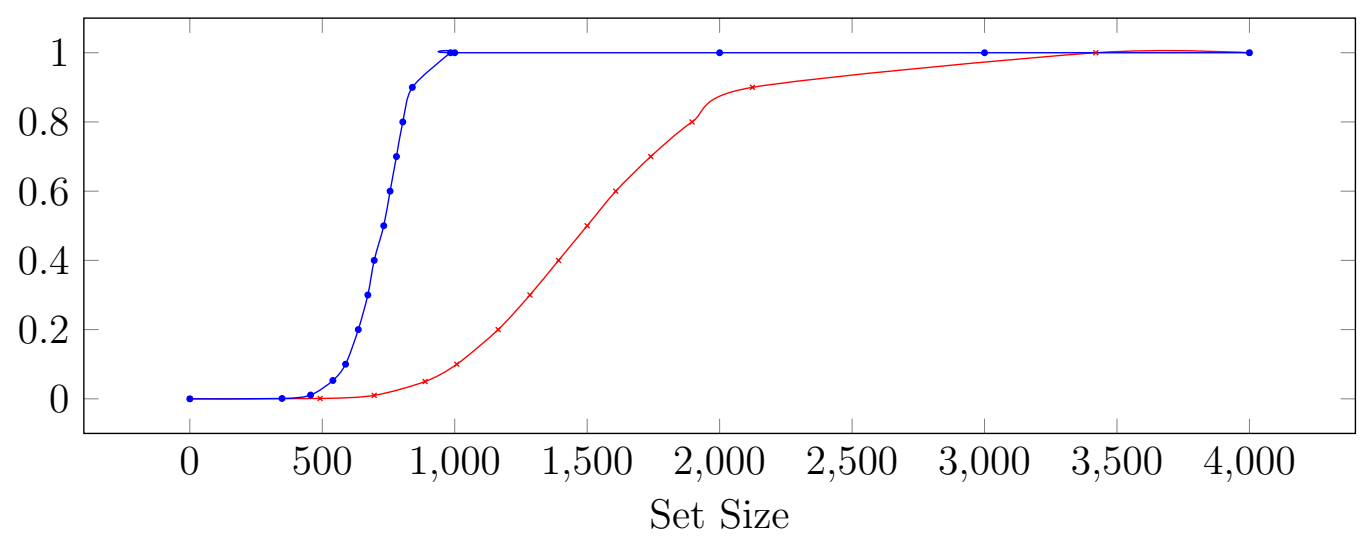

Figure 4.4: Probability of random sets to be eviction sets as a function of their size, based on our theoretical models. We use $P(C)=2^{-7}$ to represent an attacker with $4 \mathrm{~KB}$ pages in the machine from Figure 2.2. The blue-circle line shows the multinomial model for an "arbitrary" eviction set. The red-cross line shows the binomial model for an "specific" eviction set.

\section{Cost of Finding Eviction Sets}

We conclude this section by computing the cost (in terms of the expected number of memory accesses required) of finding an eviction set of size $N$ by repeatedly and independently selecting and testing candidate sets.

To this end, we model the repeated independent choice of eviction sets as a geometric distribution over the probability $p(N)$ that a candidate set of size $N$ is an eviction set. The expectation $1 / p(N)$ of this distribution captures the expected number of candidate sets that must be tested until we find an eviction set. Assuming that a test of a set of size $N$ requires $\mathcal{O}(N)$ memory accesses, as in 
Section 4.1.2, this yields an overall cost in terms of memory accesses for finding an initial eviction set of $\mathcal{O}(N / p(N))$.

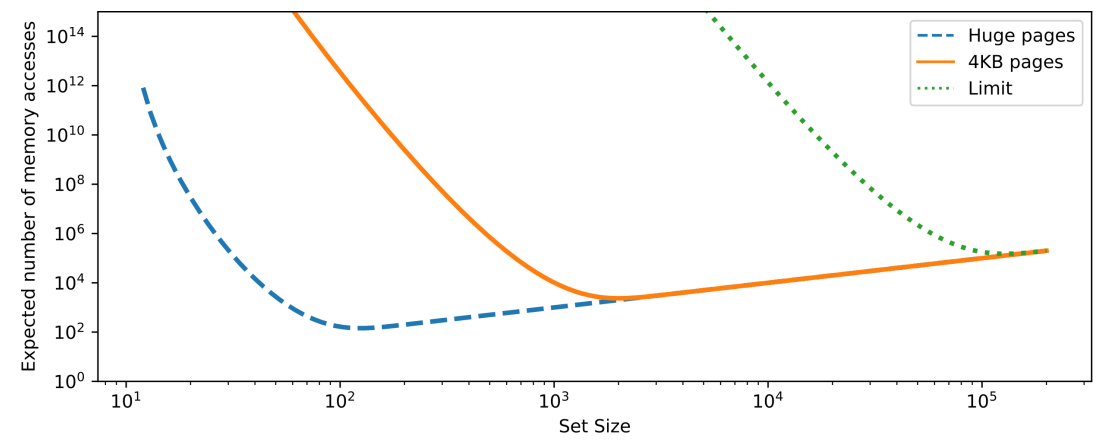

Figure 4.5: Expected number of memory accesses for finding an eviction set as a function of its size. The dashed blue line represents $P(C)=2^{-3}$, an attacker with huge pages (i.e. controls all $\gamma=10$ set index bits). The plain orange line represents $P(C)=2^{-7}$, an attacker with $4 \mathrm{~KB}$ pages (i.e. controls $\gamma=6$ ). The dotted green line represents $P(C)=2^{-13}$, an attacker w/o any control over the set index bits (i.e. $\gamma=0$ ).

Figure 4.5 depicts the cost function $N / p(N)$ for the adversaries from Example 1) for finding eviction sets for a specific address, and highlights the most favorable sizes for finding eviction sets. Since probability grows with set size, finding an eviction set of small size requires, in expectation, large number of trials. Once the probability stabilizes (i.e. the set is large enough), we start seeing the linear cost of the test.

\subsection{Algorithms for Computing Minimal Evic- tion Sets}

The probability that a set of virtual addresses forms an eviction set depends on its size, on the cache settings (e.g., associativity and number of cache sets), and on the amount of control an adversary has over the physical addresses. In particular, a small set of random virtual addresses is unlikely to be an eviction set. This motivates the two-step approach for finding minimal eviction sets in which one (1) first identifies a large eviction set, and (2) then reduces this set to its minimal core.

Previous proposals in the literature rely on this two-step approach. In this section we first present the baseline reduction from the literature, which requires $\mathcal{O}\left(N^{2}\right)$ memory accesses. We then show that it is possible to perform the reduction using only $\mathcal{O}(N)$ memory accesses, which enables dealing with much larger initial eviction sets than before. 


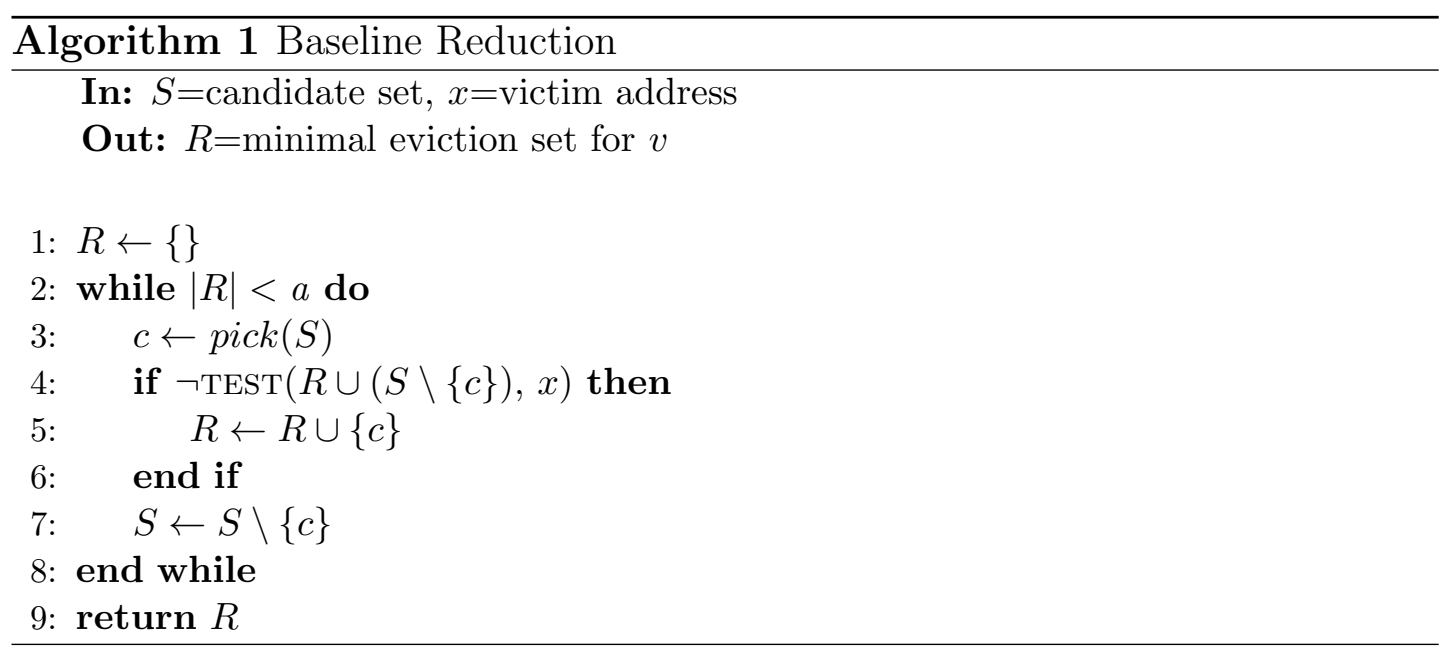

The main practical implication of this result is that finding minimal eviction sets from user (or sandboxed) space is faster than previously thought, and hence practical even without any control over the slice or set index bits. This renders countermeasures based on reducing adversary control over these bits futile.

\subsubsection{The Baseline Algorithm}

We revisit the baseline algorithm for reducing eviction sets that has been informally described in the literature. Its pseudocode is given as Algorithm 1.

Algorithm 1 receives as input a virtual address $x$ and an eviction set $S$ for $x$. It proceeds by picking an address $c$ from $S$ and tests whether $S \backslash\{c\}$ is still evicting $x$, see line 4 . If it is not (notably the if-branch), $c$ must be congruent to $x$ and is recorded in $R$, see line 5 . The algorithm then removes $c$ from $S$ in line 7 and loops in line 2 .

Note that the eviction test TEST is applied to $R \cup(S \backslash\{c\})$ in line 4, i.e. all congruent elements found so far are included. This enables scanning $S$ for congruent elements even when there are less than $a$ of them left. The algorithm terminates when $R$ forms a minimal eviction set of $a$ elements, which is guaranteed to happen because $S$ is initially an eviction set.

Proposition 1. Algorithm 1 reduces an eviction set $S$ to its minimal core in $\mathcal{O}\left(N^{2}\right)$ memory accesses, where $N=|S|$.

The complexity bound follows because $|S|$ is an upper bound for the number of loop iterations as well as on the argument size of Test 1 and hence the number of memory accesses performed during each call to TEST.

The literature contains different variants of Algorithm 1[64, 65, 69]. For example, the variant presented in [69] always puts $c$ back into $S$ and keeps iterating until $|S|=a$. This still is asymptotically quadratic, but adds some redundancy that helps to combat errors. 
If the quadratic baseline was optimal, one could think about preventing an adversary from computing small eviction sets by reducing or removing control over the set index bits, either by adding a mask via hardware [65] or a permutation layer via software [67] (see limit case in Example 1).

\subsubsection{Computing Minimal Eviction Sets for a Specific Ad- dress}

We present a novel algorithm that performs the reduction of eviction sets to their minimal core in $\mathcal{O}(N)$ memory accesses, where $N=|S|$. This enables dealing with much larger eviction sets than the quadratic baseline and renders countermeasures based on hiding the physical address futile. Our algorithm is based on ideas from threshold group testing, which we briefly introduce first.

\subsubsection{Threshold Group Testing}

Group testing refers to procedures that break up the task of identifying elements with a desired property by tests on sets (i.e. groups of those elements). Group testing has been proposed for identifying diseases via blood tests where, by testing pools of blood samples rather than individual samples, one can reduce the number of tests required to find all positive individuals from linear to logarithmic in the population size [74].

Threshold group testing [68] refers to group testing based on tests that give a negative answer if the number of positive individuals in the tested set is at most $l$, a positive answer if the number is at least $u$, and any answer if it is in-between $l$ and $u$. Here, $l$ and $u$ are natural numbers that represent lower and upper thresholds, respectively.

\subsubsection{A Linear-Time Algorithm for Computing Minimal Eviction Sets}

The key insight behind our algorithm is that testing whether a set of virtual addresses $S$ evicts $x$ (see Test 4.1 ) can actually be seen as a threshold group test for congruence with $x$, where $l=a-1$ and $u=a$. This is because the test gives a positive answer if $|[x] \cap S| \geq a$, and a negative answer otherwise. This connection allows us to leverage the following result from the group testing literature for computing minimal eviction sets.

Lemma 1 ([68]). If a set $S$ contains $p$ or more positive elements, one can identify $p$ of them using $\mathcal{O}(p \log |S|)$ threshold group tests with $l=p-1$ and $u=p$.

Proof. The idea behind Lemma 1 is to partition $S$ in $p+1$ disjoint subsets $T_{1}, \ldots, T_{p+1}$ of (approximately) the same size. A simple counting argument shows that there is at least one $j \in\{1, \ldots, p+1\}$ such that $S \backslash T_{j}$ is still an eviction 


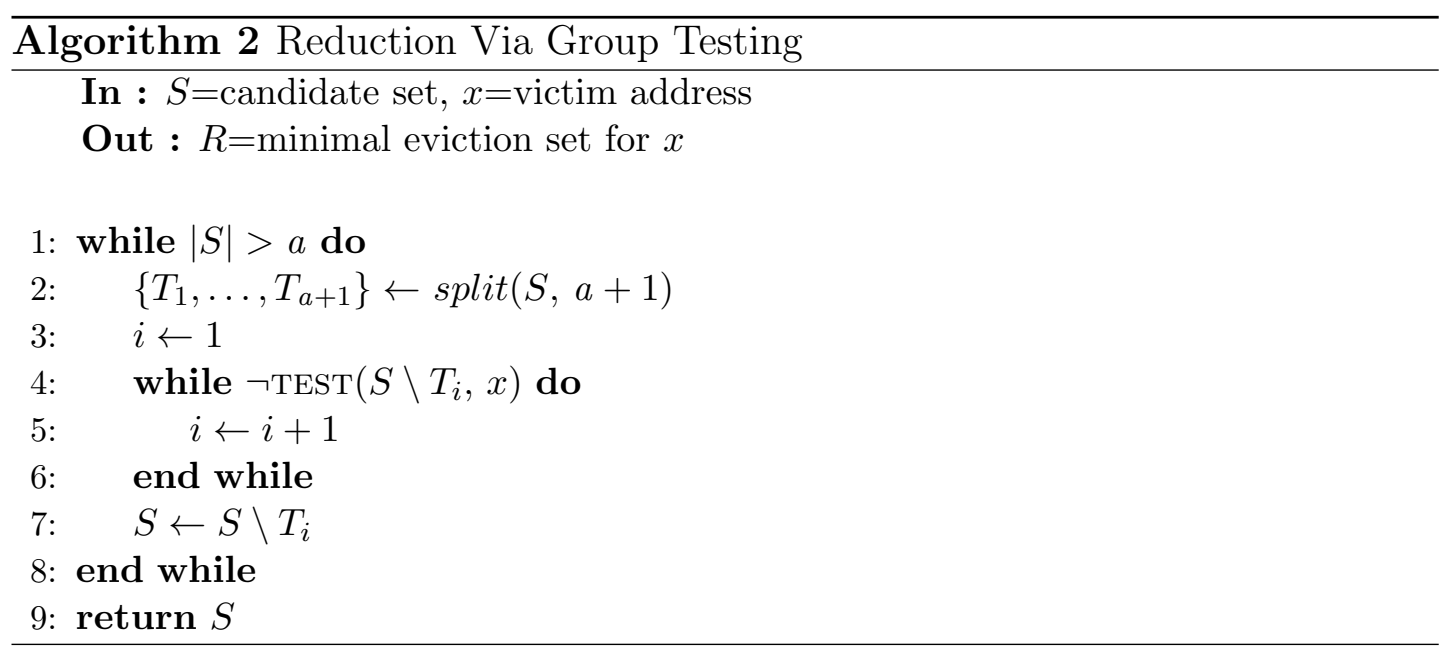

set. One identifies such a $j$ by group tests and repeats the procedure on $S \backslash T_{j}$. The logarithmic complexity is due to the fact that $\left|S \backslash T_{j}\right|=|S| \frac{p}{p+1}$, i.e. each iteration reduces the eviction set by a factor of its size, rather by than a constant as in Algorithm 1.

Algorithm 2 computes minimal eviction sets based on this idea. Note that Lemma 1 gives a bound on the number of group tests. For computing eviction sets, however, the relevant complexity measure is the total number of memory accesses made, i.e. the sum of the sizes of the sets on which tests are performed. We next show that, with this complexity measure, Algorithm 2 is linear in the size of the initial eviction set.

Proposition 2. Algorithm 2 with Test 1 reduces an eviction set $S$ to its minimal core using $O\left(a^{2} N\right)$ memory accesses, where $N=|S|$.

Proof. The correctness of Algorithm 2 follows from the invariant that $S$ is an eviction set and that it satisfies $|S|=a$ upon termination, see Lemma 1. For the proof of the complexity bound observe that the number of memory accesses performed by Algorithm 2 on a set $S$ of size $N$ follows the following recurrence.

$$
T(N)=T\left(N \frac{a}{a+1}\right)+N \cdot a
$$

for $N>a$, and $T(a)=a$. The recurrence holds because, on input $S$, the algorithm applies threshold group tests on $a+1$ subsets of $S$, each of size $N-\frac{N}{a+1}$. The overall cost for the split and the tests is $N \cdot a$. The algorithm recurses on exactly one of these subsets of $S$, which has size $N \frac{a}{a+1}$. From the Master theorem [75] it follows that $T(N) \in \Theta(N)$.

Direct Proof of Proposition 2 For completeness, we also include a direct proof. 
In the worst case, we access $a+1$ different $a$-subsets groups of size $\frac{n}{a+1}$ each, and safely discard $\frac{n}{a+1}$ elements that are not part of the minimal eviction set. We first express recurrence (4.2) as a summation

$$
T(n)=a n+a n\left(\frac{a}{a+1}\right)+a n\left(\frac{a}{a+1}\right)^{2}+\cdots+a n\left(\frac{a}{a+1}\right)^{k}
$$

Our termination condition is $n\left(\frac{a}{a+1}\right)^{k}<a$, meaning that we already have an minimal eviction set. By using the logarithm we can set the exponent of the last iteration as $k=\log _{a /(a+1)} a / n$, which allows us to define the function as a geometric progression

$$
T(n)=a \sum_{i=1}^{i=k} n\left(\frac{a}{a+1}\right)^{i-1}=\frac{a n\left(1-\left(\frac{a}{a+1}\right)^{\log _{a /(a+1)} a / n}\right)}{1-\frac{a}{a+1}}
$$

The logarithm in the exponent cancels out, and we obtain

$$
T(n)=\frac{a n\left(1-\frac{a}{n}\right)}{1-\frac{a}{a+1}}=a(n-a)(a+1)=a^{2} n+a n-a^{3}-a^{2}
$$

We ignore the effect of the ceiling operator required in a real implementation, where $n$ is always an integer. It can be shown that this error is bounded by a small factor of our $k$, so we consider it negligible.

\subsubsection{Computing Minimal Eviction Set for an Arbitrary Address}

The Algorithms presented so far compute minimal eviction sets for a specific virtual address $x$. We now consider the case of computing minimal eviction sets for an arbitrary address. This case is interesting because, as shown in Section 4.1.3, a set of virtual addresses is more likely to evict any arbitrary address than a specific one. That is, in scenarios where the target address is not relevant, one can start the reduction with smaller candidate sets.

The key observation is that both Algorithm 1 and Algorithm 2 can be easily adapted to compute eviction sets for an arbitrary address. This only requires replacing the eviction test for a specific address (Test 4.1) by an eviction test for an arbitrary address (Test 4.3).

Proposition 3. Algorithm 1, with Test 4.3 for an arbitrary eviction set, reduces an eviction set to its minimal core in $\mathcal{O}\left(N^{2}\right)$ memory accesses, where $N=|S|$.

Proposition 4. Algorithm 2 with Test 4.3 reduces an eviction set to its minimal core in $\mathcal{O}(N)$ memory accesses, where $N=|S|$.

The complexity bounds for computing eviction sets for an arbitrary address coincide with those in Proposition 1 and 2 because Test 4.1 and Test 4.3 are both linear in the size of the tested set. 


\subsubsection{Computing Minimal Eviction Sets for Many Virtual Addresses}

We now discuss the case of finding eviction sets for a large number of cache sets. For this we assume a given pool $P$ of virtual addresses, and explain how to compute minimal eviction sets for all the eviction sets that are contained in $P$. For large enough $P$ the result can be a set of eviction sets for all virtual addresses.

The core idea is to use a large enough subset of $P$ and reduce it to a minimal eviction set $S$ for an arbitrary address, say $x$. Use $S$ to build a test $\operatorname{Test}((S \backslash\{x\}) \cup\{y\}, x)$ for individual addresses $y$ to be congruent with $x$. Use this test to scan $P$ and remove all elements that are congruent with $x$. Repeat the procedure until no more eviction sets are found in $P$. With a linear reduction using Algorithm 2, a linear scan, and a constant number of cache sets, this procedure requires $\mathcal{O}(|P|)$ memory accesses to identify all eviction sets in $P$.

Previous work [69] proposes a similar approach based on the quadratic baseline reduction. The authors leverage the fact that, on earlier Intel CPUs, given two congruent physical addresses $x \simeq y$, then $x+\Delta \simeq y+\Delta$, for any offset $\Delta<2^{\gamma}$. This implies that, given one eviction set for each of the $2^{c-\gamma}$ page colors, one can immediately obtain $2^{\gamma}-1$ others by adding appropriate offsets to each address. Unfortunately, with unknown slicing functions this only holds with probability $2^{-s}$, what increases the attacker's effort. Our linear-time algorithm helps scaling to large numbers of eviction sets under those conditions.

Another solution to the problem of finding many eviction sets has been proposed in [64]. This solution differs from the two-step approach in that the algorithm first constructs a so-called conflict set, which is the union of all minimal eviction sets contained in $P$, before performing a split into the individual minimal eviction sets. The main advantage of using conflict sets is that, once a minimal eviction set is found, the conflict set need not be scanned for further congruent addresses.

\subsection{Evaluation}

In this section we perform an evaluation of the algorithms for computing minimal eviction sets we have developed in Section 4.2. The evaluation complements our theoretical analysis along two dimensions:

Robustness The theoretical analysis assumes that tests for eviction sets always return the correct answer, which results in provably correct reduction algorithms. In this section we analyze the robustness of our algorithms in practice. In particular, we study the influence of factors that are outside of our model, such as adaptive cache replacement policies and TLB activity. We identify conditions under which our algorithms are almost perfectly reliable, as well as conditions 
under which their reliability degrades. These insights can be the basis of principled countermeasures against, or paths forward for improving robustness of, algorithms for finding eviction sets.

Execution time The theoretical analysis captures the performance of our algorithms in terms of the number of memory accesses. As for the case of correctness, the real execution time is influenced by factors that are outside of our model, such as the total number of cache and TLB misses, or the implementation details. In our empirical analysis we show that the number of memory accesses is in fact a good predictor for the asymptotic real-time performance of our algorithms.

\subsubsection{Design of our Analysis}

Implementation We implemented the tests and algorithms described in Sections 4.1 .2 and 4.2 as a command line tool, which can be parameterized to find minimal eviction sets on different platforms. All of our experiments are performed using the tool. The source code is available at: https://github.com/cgvwzq/ evsets.

Analyzed Platforms We evaluate our algorithms on two different CPUs running Linux 4.9:

1. Intel i5-6500 $4 \times 3.20 \mathrm{GHz}$ (Skylake family), $16 \mathrm{~GB}$ of RAM, and a $6 \mathrm{MB}$ LLC with 8192 12-way cache sets. Our experiments indicate that only 10 bits are used as set index on this machine, we hence conclude that each core has 2 slices. Following our previous notation, i.e.: $a_{s k y}=12, c_{s k y}=$ $10, s_{s k y}=3, \ell_{s k y}=6$.

2. Intel i7-4790 8 x $3.60 \mathrm{GHz}$ GHz (Haswell family), $8 \mathrm{~GB}$ of RAM, and a $8 \mathrm{MB}$ LLC with 8192 16-way cache sets. This machine has 4 physical cores and 4 slices. Following our previous notation, i.e.: $a_{\text {has }}=16, c_{\text {has }}=11, s_{\text {has }}=$ $2, \ell_{\text {has }}=6$.

We emphasize that all experiments run on machines with user operating systems (with a default window manager and background services), default kernel, and default BIOS settings.

Selection of Initial Search Space We first allocate a big memory buffer as a pool of addresses from where we can suitably chose the candidate sets (recall Section 4.1.3). This choice is done based on the adversary's capabilities (i.e., $\gamma$ ), for example, by collecting all addresses in the buffer using a stride of $2^{\gamma+\ell}$, and then randomly selecting $N$ of them. With this method, we are able to simulate any amount of adversary control over the set index bits, i.e. any $\gamma$ with $\gamma<p-\ell$. 
Isolating and Mitigating Interferences We identify ways to isolate two important sources of interference that affect the reliability of our tests and hence the correctness of our algorithms:

- Adaptive Replacement Policies: Both Skylake and Haswell employ mechanisms to adaptively switch between undocumented cache replacement policies. Our experiments indicate that Skylake keeps a few fixed cache sets (for example, the cache set zero) that seem to behave as PLRU and match the assumptions of our model. Targeting such sets allows us to isolate the effect of adaptive and unknown replacement policies on the reliability of our algorithms.

- Translation Lookaside Buffers: Performing virtual memory translations during a test results in accesses to the TLB. An increased number of translations can lead to an increased number of TLB misses, which at the end trigger page walks. These page walks result in implicit memory accesses that may evict the target address from the cache, even though the set under test is not an eviction set, i.e. it introduces a false positive. TLB misses also introduce a noticeable delay on time measurements, what has been recently discussed in a concurrent work [76]. We isolate these effects by performing experiments for pages of $4 \mathrm{~KB}$ on huge pages of $2 \mathrm{MB}$, but under the assumption that, as for $4 \mathrm{~KB}$ pages, only $\gamma=6$ bits of the set index are under attacker control.

We further rely on common techniques from the literature to mitigate the influence of other sources of interference:

- For reducing the effect of hardware prefetching we use a linked list to represent eviction sets, where each element is a pointer to the next address. This ensure that all memory accesses loads are executed in-order. We further randomize the order of elements.

- For reducing the effect of jitter, we perform several time measurements per test and compare their average value with a threshold. In our experiments, $10-50$ measurements are sufficient to reduce the interference of context switches and other spurious events. More noisy environments (e.g. a web browser) may require larger numbers.

\subsubsection{Evaluating Robustness}

We rely on two indicators for the robustness of our tests and reduction algorithms:

- The eviction rate, which is the relative frequency of our tests returning true on randomly selected sets of fixed size.

- The reduction rate, which we define as the relative frequency of our reduction succeeding to reduce randomly selected sets of fixed size to a minimal eviction set. 
Here, a reduction is successful if the elements it returns are congruent, i.e., they coincide on the set bits and on the slice bits. For this check we rely on the reverse engineered slice function for Intel CPUs [77].

With perfect tests (and hence correct algorithms), both the eviction rate and the reduction rate should coincide with the theoretical prediction given in Section 4.1. Our analysis hence focuses on deviations of the eviction and reduction rate from these predictions.
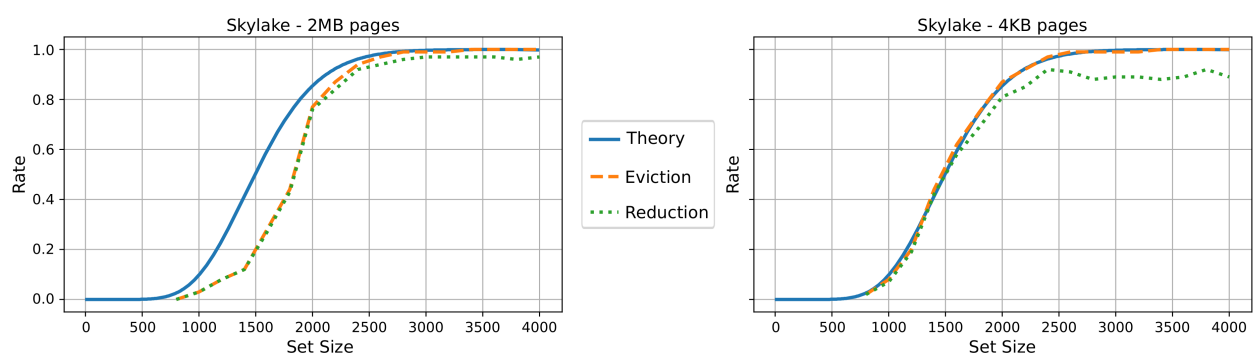

Figure 4.6: Skylake: Eviction for specific address $x$ on cache set zero, compared to our binomial model. Each point is the average of 1000 reductions for sets of $N$ randomly chosen addresses.

Experimental Results The experimental results for eviction and reduction for a specific address $x$ are given in Figures 4.6 and 4.7a (for Skylake), and Figure 4.8a (Haswell). The results for arbitrary addresses are given in Figures 4.7b and 4.8b (for Skylake and Haswell, respectively). We highlight the following findings:

- Analysis under idealized conditions We first analyze our test and reduction algorithms under idealized conditions, where we use the techniques described in Section 4.3.1 to mitigate the effect of TLBs, complex replacement policies, prefetching, and jitter. Figure 4.6 illustrates that, under these conditions, eviction and reduction rates (Test 4.1 and Algorithm 2) closely match. Moreover, eviction and reduction rates closely match the theoretical prediction for small pages.

For huge pages, however, eviction and reduction rates remain below the theoretical prediction, see Figure 4.6. We attribute this to the fact that, using explicit allocations, huge pages are chosen from a pre-allocated pool of physical pages that usually resides in a fixed zone. This limits the uniformity of the more significant bits and deviates from our uniform modeling.

- Effect of the cache replacement policies. Our experimental results show that the eviction and reduction rates decrease significantly on Haswell (Figure 4.8a), and on Skylake when targeting a cache set (Figure 4.7a) different from zero. The effect is also visible in the evaluation of algorithms for finding an arbitrary eviction set (see Figures $4.7 \mathrm{~b}$ and $4.8 \mathrm{~b}$ ). 

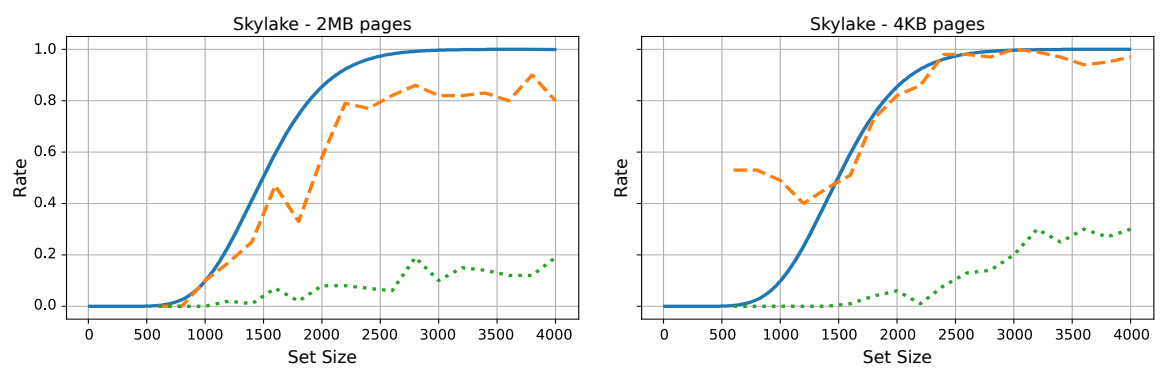

(a) Eviction and reduction rates for an specific address $x$ targeting cache set $\mathbf{1 0}$, compared to our binomial model.
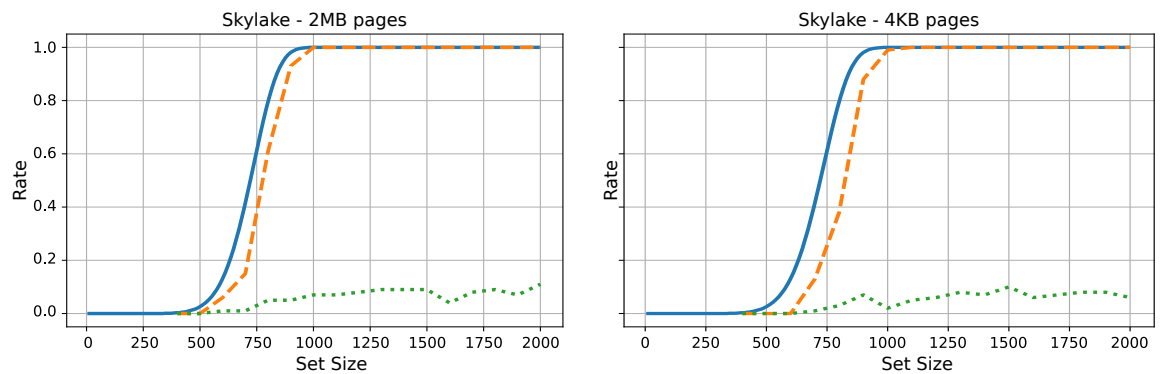

(b) Eviction and reduction rates for an arbitrary address, compared to our multinomial model.

Figure 4.7: Experiments on Skylake. Each point is the average of 100 reductions for sets of $N$ randomly chosen addresses.

The decrease seems to be caused by two factors: the replacement policy of the targeted cache sets does not match our models; and the targeted cache set are influenced by accesses to other sets in the cache. We provide further evidence of this effect in Section 4.4.

- Effect of TLB thrashing. Virtual memory translations are more frequent with small pages than with huge pages, which shows in our experiments: The eviction rate lies above the theoretical prediction, in particular for large sets, which shows the existence of false positives. In contrast, the reduction rate falls off. This is because false positives in tests cause the reduction to select sets that are not eviction sets, which leads to failure further down the path.

Modern CPUs have very distinct TLBs implementations. In particular, modern Intel CPUs implement different buffers for data (dTLB) and instructions (iTLB), a second level TLBs (sTLB) with larger capacity, and different TLBs for each PT level. Table 4.1 shows a summary of TLB parameters for Haswell and Skylake families: 

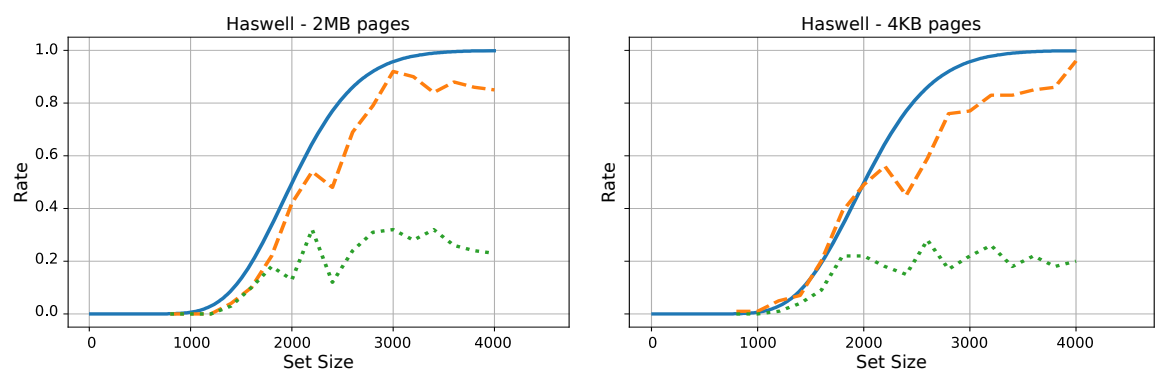

(a) Eviction and reduction rates for an specific address $x$, compared to our binomial model.
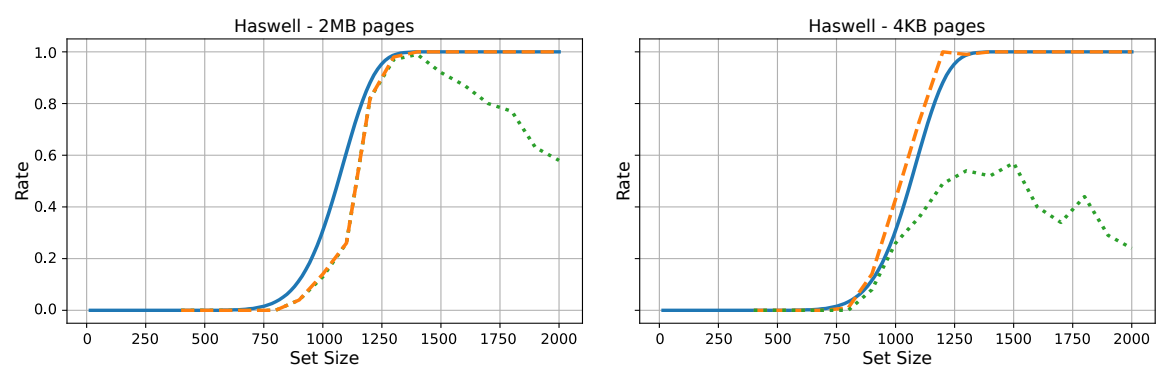

(b) Eviction and reduction rates for an arbitrary address, compared to our multinomial model.

Figure 4.8: Experiments on Haswell. Each point is the average of 100 reductions for sets of $N$ randomly chosen addresses.

\begin{tabular}{l|c|r} 
& Haswell & Skylake \\
\hline iTLB 4K & 128 entries; 4-way & 128 entries; 8-way \\
iTLB 2M/4M & 8 entries; full & 8 entries; full \\
\hline dTLB 4K & 64 entries; full & 64 entries; 4-way \\
dTLB 2M/4M & 32 entries; 4-way & 32 entries; 4-way \\
dTLB 1G & 4 entries; 4-way & 4 entries; full \\
\hline sTLB 4K/2M & 1024 entries; 8-way & 1536 entries; 4-way \\
sTLB 1G & - & 16 entries; 4-way \\
\hline
\end{tabular}

Table 4.1: TLB implementation information for Haswell and Skylake microarchitectures. Extracted from the Intel's Architectures Optimization Manual [2].

The effect is clearly visible in Figure 4.6, where we compare the results on small pages with those on huge pages for cache set zero on Skylake. We observe that the reduction rate on small pages declines for $N>1500$, which, as Table 4.1 shows, coincides with the TLB capacity of Skylake of 1536 entries.

The effect is also visible in Figure $4.8 \mathrm{~b}$, where we attribute the strong decline of the reduction rate after $N>1000$ (Haswell's TLB capacity is 1024 entries) to implicit memory accesses having a greater chance to be an 
eviction set for Haswell's adaptive replacement policy. In the rest of figures the effect is overlaid with interferences of the replacement policy. However, Figure $4.7 \mathrm{~b}$ shows that with large TLBs, and for most reasonable values of $N$, the effect of TLB thrashing is negligible.

\subsubsection{Evaluating Performance}

We evaluate the performance of our novel reduction algorithm and compare it to that of the baseline from the literature. For this, we measure the average time required to reduce eviction sets of different sizes to their minimal core. We first focus on idealized conditions that closely match the assumptions of the theoretical analysis in Section 4.2.

To put the performance of the reduction in context, we also evaluate the effort that is required for finding an initial eviction set to reduce. For this, we consider attackers with different capabilities to control the set index bits, based on huge pages $(\gamma=10), 4 \mathrm{~KB}$ pages $(\gamma=6)$, and with no control over the set index bits $(\gamma=0)$.

Together, our evaluation gives an account of how the performance gains of our novel reduction algorithm affect the overall effort of computing minimal eviction sets.

Experimental Results The results of the evaluation of the reduction for a specific address on Skylake are given in Figure 4.9. We focus on cache set zero to mitigate the effect of the replacement policy, and we mitigate the influence of TLBs and prefetching as described in Section 4.3.1. ${ }^{1}$

Each data point is based on the average execution time of 10 successful reductions. The sizes of the initial sets (x-axis) are chosen to depict the range where finding an initial eviction set does not require picking a too large number of candidate sets (depicted by the green bars). For a more systematic choice of the initial set size see the discussion below.

We highlight the following observations:

- The slope of the orange curve clearly illustrates the quadratic growth of execution time of the naive reduction, whereas the blue curve shows the linear growth of our novel algorithms. The absolute values account for constant factors such as the 50 time measurements per test, and the overhead due to metrics collection.

- For large set sizes, our novel reduction clearly outperforms the quadratic baseline. For example, for sets of size 3000, we already observe a performance improvement of a factor of 10 , which shows a clear practical advantage. For small set sizes, this practical advantage seems less relevant.

\footnotetext{
${ }^{1}$ In the limit case the stride of $64 \mathrm{~B}$ makes inferences by prefetching prohibitive even with a randomized order, which is why we disable hardware prefetchers using wrmsr -a 0x1a4 15.
} 

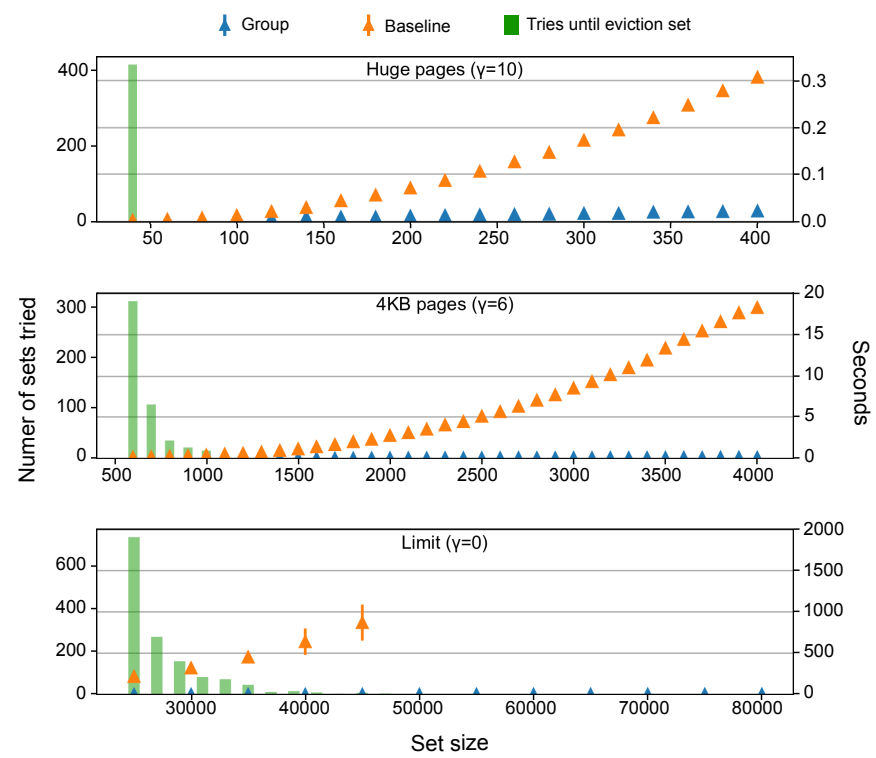

Figure 4.9: The vertical green bars (left axis) depict the average number of times one needs to pick a set of addresses for finding an eviction set. Triangles (right axis) show time in seconds: blue depicts the average execution time of group test reductions; orange depicts the average execution time of baseline reductions. Different plots illustrate attackers with huge pages, 4KB pages, and w/o any control over the set index bits, respectively.

For such sizes, however, the number of repetitions required until find a real eviction set grows, as illustrated by the green bars. For the total cost of finding an eviction set, both effects need to be considered in combination.

Optimal Choice of the Initial Set Size For evaluating the cost of first identifying and then reducing eviction sets, we rely on an expression for the overall number of memory accesses required for finding a minimal eviction set. This expression is the sum of the expected number $\frac{N}{p(N)}$ of memory accesses for finding an eviction set, see Section 4.1.3, and of the memory accesses for the respective reductions $N^{2}$ and $N$, see Propositions 1 and 2. Based on this expression, we compute the optimal set sizes (from an attacker's perspective) for the linear and the quadratic reductions. We use these sizes as an approximation of the optimal use of each reduction algorithm in the overall pipeline, and we evaluate their execution time on sets of this size.

Table 4.2 shows the comparison of the linear and the quadratic reductions on sets of optimal size for three different attackers: with huge pages, with $4 \mathrm{~KB}$ pages, and in the limit.

We highlight the following observations:

- For huge pages, computing eviction sets is cheap, and the linear-time re- 


\begin{tabular}{c|c|c|c|c|c}
\multicolumn{2}{l|}{} & \multicolumn{2}{c|}{ Baseline } & \multicolumn{2}{c}{ Group Testing } \\
\hline Attacker & $P(C)$ & $N$ & Time & $N$ & Time \\
\hline HP $(\gamma=10)$ & $2^{-3}$ & 62 & $0.005 \mathrm{~s}$ & 62 & $0.004 \mathrm{~s}$ \\
$4 \mathrm{~KB}(\gamma=6)$ & $2^{-7}$ & 662 & $0.179 \mathrm{~s}$ & 862 & $0.023 \mathrm{~s}$ \\
Limit $(\gamma=0)$ & $2^{-13}$ & 26650 & $218.651 \mathrm{~s}$ & 53300 & $1.814 \mathrm{~s}$ \\
\hline
\end{tabular}

Table 4.2: $N$ shows the optimal set sizes for different attackers $(\gamma$ bits) on Skylake $(a=12)$ using 50 time measurements per test. Time shows the average execution time of our implementations of Algorithm 1 (baseline) and Algorithm 2 (group testing) under ideal conditions.

duction does not lead to a practical advantage.

- For small pages, the linear-time reduction improves the cost of computing eviction sets by a factor of more than 7 . This is a significant advantage in practice, as it can make attacks more stealthy and robust against timing constraints.

- For the limit case, the linear-time reduction improves over the quadratic baseline by more than two orders of magnitude.

\subsubsection{Performance in Practice}

In this section we give examples of the performance benefits of our reduction algorithms in real-world scenarios, i.e., in the presence of TLB noise and adaptive replacement policies.

We implement two heuristics to counter the resulting sub-optimal reduction rates (see Section4.3.1): repeat-until-success, where we pick a new set and start over after a failed reduction; and backtracking, where at each level of the computation tree we store the elements that are discarded, and, in case of error, go back to a parent node on which the test succeeded to continue the computation from there. For more details we refer to our open-source implementation.

For comparing the performance of the reduction algorithms in the context of these heuristics, we follow the literature and focus on initial set sizes that guarantee that the initial set is an eviction set with high probability. This is because a real-world attacker is likely to forgo the complications of repeatedly sampling and directly pick a large enough initial set.

The following examples provide average execution times (over 100 samples) for different attackers on randomly selected target cache sets. Skylake $(a=12)$ using 10 time measurements per test.

- For finding eviction sets with huge pages, previous work [78] suggests an initial set size of $N=192$ which, according to our binomial model (see Section 4.1.3), yields a probability of sets to be evicting close to 1 . For this size, the baseline reduction takes 0.014 seconds, while the group-testing reduction takes 0.003 seconds, i.e. our algorithm improves the baseline by a factor of 5 . 
- For finding minimal eviction sets with $4 K B$ pages, previous work [69] suggests an initial set size of $N=8192$, which amounts to the size of LLC times the number of slices. We choose an initial set size of $N=3420$ for our comparison, which according to our model provides a probability of being an eviction set close to 1 . For this $N$, the baseline reduction takes 5.060 seconds, while the group-testing reduction takes 0.245 seconds, i.e. our algorithm improves the baseline by a factor of 20 . Finding all minimal eviction sets (for a fixed offset) within this buffer ${ }^{2}$ requires more than 100 seconds with the baseline algorithm. With group testing, the same process takes only 9.339 seconds, i.e. it improves by a factor of 10 .

\subsubsection{Summary}

In summary, our experiments show that our algorithms improve the time required for computing minimal eviction sets by factors of 5-20 in practical scenarios. Moreover, they show that finding minimal eviction sets from virtual (or sandboxed) memory space is fast even without any control over the slice or set index bits, rendering countermeasures based on masking these bits futile.

\subsection{A Closer Look at the Effect of Modern Cache Replacement Policies}

There are several features of modern microarchitectures that are not captured in our model and that can affect the effectiveness of our algorithms, such as adaptive and randomized replacement policies, TLBs, prefetching, etc. The evaluation of Section 4.3 shows that the influence of prefetching can be partially mitigated by an adversary, and that the influence of TLBs is not a limiting factor in practice. The role of the cache replacement policy is less clear.

In this section, we take a closer look at the role of modern cache replacement policies in computing minimal eviction sets. As discussed in Section 2.2, post Sandy Bridge architectures boast replacement policies with features such as adaptivity or thrashing-resistance. With such features, accessing a set of $a$ addresses that are congruent with $[x]$ is neither necessary nor sufficient for evicting $x$, which introduces a two-sided error (false positives and false negatives) in our tests for congruence. We first explain the key mechanisms that lead to this error, before we experimentally analyze its influence on Skylake and Haswell.

\footnotetext{
${ }^{2}$ We empirically observe that on Skylake, this size is sufficient to contain eviction sets for most of the 128 different cache sets for a fixed offset.
} 


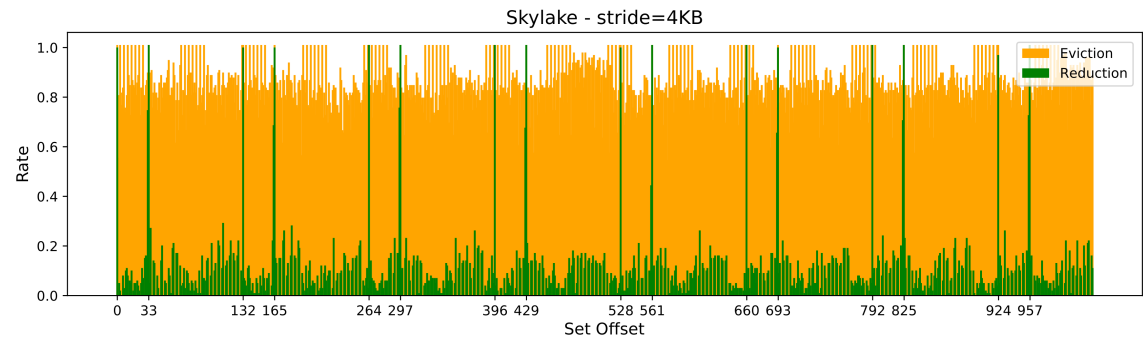

Figure 4.10: Skylake's eviction and reduction rates per set index. With a stride of $4 \mathrm{~KB}$ and a total of 4000 addresses (most of them non-congruent). The number of sets in-between two leaders is either 32 or 98 . We rely on huge pages to precisely control the target's set index bits.

\subsubsection{Adaptive Replacement Policies}

Adaptive cache replacement policies [79] dynamically select the replacement policy depending on which one is likely to be more effective on a specific load. For this, they rely on so-called leader sets that implement different policies. A counter keeps track of the misses incurred on the leaders and adapts the replacement policy of the follower sets depending on which leader is more effective at the moment. There are different ways for selecting the leaders: a static policy in which the leader sets are fixed; and a rand-runtime policy that randomly selects different leaders every few millions instructions.

A previous study indicates that the replacement mechanism used in Ivy Bridge is indeed adaptive, with static leader sets [25]. To the best of our knowledge, there is no detailed study of replacement mechanisms on more recent generations of Intel processors such as Haswell, Broadwell, or Skylake, but there are mentions of high-frequency policy switches on Haswell and Broadwell CPUs as an obstacle for prime+probe attacks [69].

We perform different experiments to shed more light on the implementations of adaptivity in Skylake and Haswell, and on their influence on computing minimal eviction sets. To this end, we track eviction and reduction rates (see Section 4.3) for each of the set indexes individually

1. on arbitrary eviction sets

2. on eviction sets in which all addresses are partially congruent.

In the second case, the reduction uses only addresses belonging to a single cache set per slice. Assuming independence of cache sets across slice, a comparison with the first case allows us to identify the influence across cache sets. For both experiments we rely on huge pages in order to precisely control the targeted cache set and reduce the effect of the TLB, see Section 4.3.1. 


\subsubsection{Evaluating the Effect of Adaptivity}

The results for reducing arbitrary eviction sets on Skylake are given in Figure 4.10, the results for Haswell are given in Figure 4.11. We focus on initial eviction sets of size $N=4000$ (but observe similar results for other set sizes). We highlight the following findings:

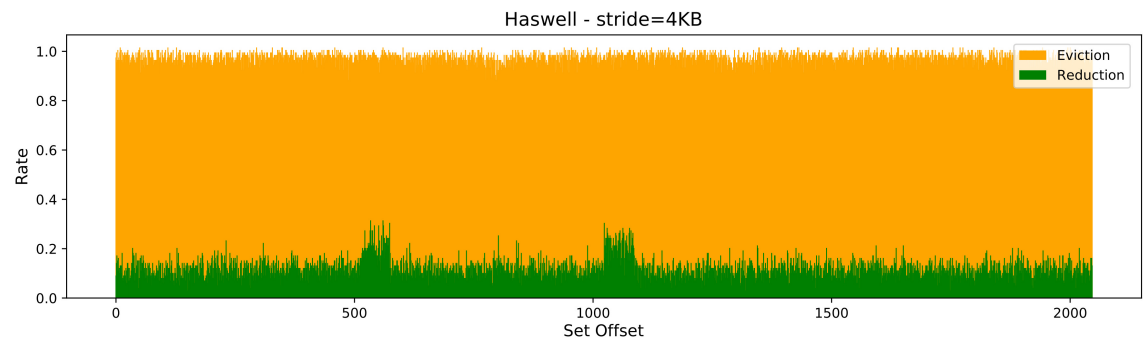

Figure 4.11: Haswell's eviction and reduction rates per set index. With a stride of $4 \mathrm{~KB}$ and a total of 4000 addresses (most of them non-congruent).

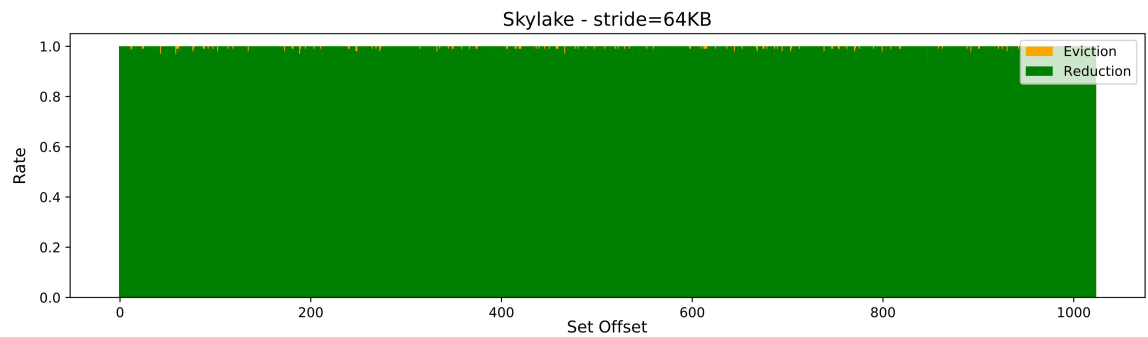

(a) Skylake's eviction and reduction rates per set index, based on a stride of $64 \mathrm{~KB}$ (only partially congruent addresses).

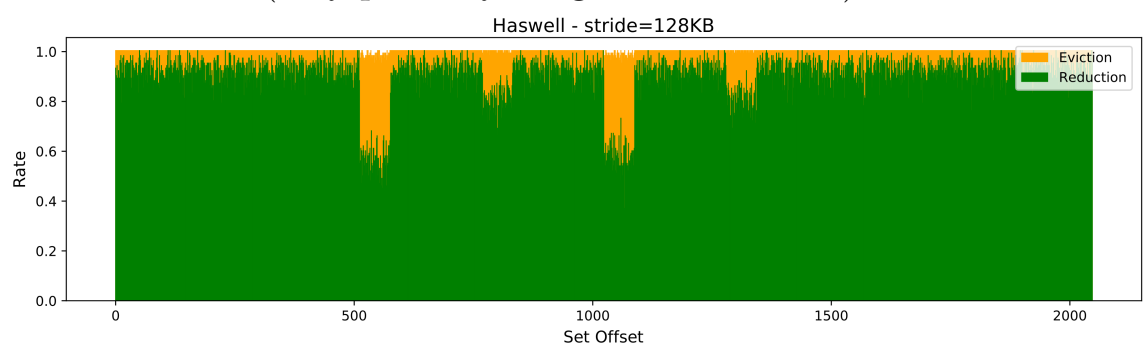

(b) Haswell's eviction rate and reduction rate per set index, based on a stride of $128 \mathrm{~KB}$ (only partially congruent addresses).

Figure 4.12: Eviction rate and reduction rate per set index for initial sets of 4000 partially congruent addresses.

- Skylake seems to implement an adaptive replacement mechanism with static leader sets, much like Ivy Bridge. In particular, we identify a subset of 16 (out of 1024 per slice) sets where the reduction rate is consistently above 


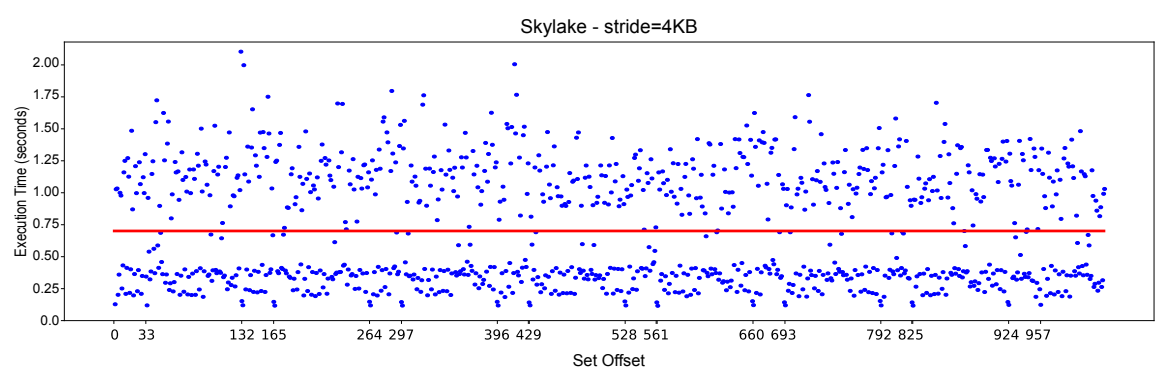

Figure 4.13: Skylake's total execution time per set index using backtracking and repeat-until-success. Average time over 100 samples, all of them successful. Stride of $4 \mathrm{~KB}$ (simulate adversary) and initial set of 4000 addresses (most of them noncongruent). The lowest execution times (below $0.12 \mathrm{~s}$ ), correspond to sets with higher reduction rate. Horizontal line shows the overall average execution time.

95\% and where tests consistently evict the target address according to our model (i.e. without false positives and false negatives). On the follower sets the reduction rate quickly falls off despite a high eviction rate, indicating that the test can produce both false positives and false negatives.

- In contrast to Skylake, on Haswell we are not able to identify any leader sets with consistently high reduction rates, which suggests a dynamic leader selection policy is in place.

The results of our reductions on partially congruent eviction sets on Haswell and Skylake are given in Figure 4.12. They show that eviction and reduction rates are close to the predicted optimum. This improves over the reduction rate in Figure 4.10 and 4.11, and indicates a strong interference on the eviction test when accessing neighboring cache sets. In particular, we observe that the robustness of the reduction increases with the proportion of partially congruent addresses in the initial eviction set.

Finally, Figure 4.13 depicts the average execution time, including the overhead of the backtracking heuristic, of finding a minimal eviction set for each cache set index. A lower reduction rate implies a higher number of errors, and hence more backtracking steps and a longer execution time. This effect is visible when comparing with Figure 4.10: cache sets with the highest reduction rates have the lowest execution times. ${ }^{3}$

\footnotetext{
${ }^{3}$ The plot also shows two different clusters of execution times, for which we currently lack a satisfying explanation.
} 


\subsection{Related Work}

Computing minimal, or at least small, eviction sets provides an essential primitive for removing or placing arbitrary data in the cache, which is essential for LLC cache attacks (Prime+Probe [64], Evict+Reload [80], etc.), for DRAM fault attacks (such as Rowhammer [81, 6], which break the separation between security domains), for memory-deduplication attacks (such as VUSION [82]), as well as for the recent Meltdown [14] and Spectre [13] attacks (which use the cache to leak data across boundaries and to increase the number of speculatively executed instructions).

Gruss et al. [66] already identified dynamic and static approaches for finding eviction sets. The dynamic method uses timing measurements to identify colliding addresses without any knowledge about the LLC slicing hash function or the physical addresses; whereas the static method use the reverse engineered hash and (partial) information about the physical addresses to compute eviction sets. In practice, most attacks [78] rely in a hybrid approach, producing a partially congruent set of addresses with static methods, and pruning or reducing the results with a dynamic method (mostly variants of Algorithm 1). We review some of the most relevant approaches:

Fully static, without slicing In CPUs without slicing (such as ARM) it is possible to find eviction sets directly using the information from the pagemap interface. Lipp et al. [83] explore how to perform Prime+Probe, Evict+Reload, and other cross-core cache attacks on ARM. Fortunately, Google patched Android in March $2016^{4}$, and now it requires root privileges to disclose physical addresses, difficulting the task of finding eviction sets.

Static/Dynamic with huge pages Liu et al. [64] and Irazoqui et al. [65], in their seminal work on attacks against LLC, rely on 2MB huge pages to circumvent the problem of mapping virtual addresses into cache sets. They are the first to propose this method.

Gruss et al. [66] present the first rowhammer attack from JavaScript. To achieve this, they build eviction sets thanks to 2MB huge pages (provided by some Linux distributions with transparent huge pages support).

On the other hand, more sophisticated cache attacks from Intel's SGX [84] rely on the predictable physical allocation of large arrays within SGX enclaves, and on the information extracted from another side-channel in DRAM row's buffers.

Sandboxed environments without huge pages Oren et al. [69] present an extension to Liu et al.'s work, carrying out the first cache attack from JavaScript, where regular $4 \mathrm{~KB}$ pages are used and pointers are not directly available. It

\footnotetext{
${ }^{4}$ Android patch: https://source.android.com/security/bulletin/2016-03-01
} 
exploits the knowledge of browser's page aligned allocation for large buffers to construct an initial set with identical page offset bits. Then they leverage the clever technique described in Section 4.2.4 for further accelerating the process of finding other eviction sets.

Dedup Est Machina [72] also implements a JavaScript rowhammer attack, but this time targeting Microsoft Edge on Windows 10. Interestingly, they can not rely on large pages, since Microsoft Edge does not explicitly request them. However, they discover that the Windows kernel dispenses pages for large allocations from different physical memory pools that frequently belong to the same cache sets. Thereby, they are able to efficiently find eviction sets (not minimal) by accessing several addresses that are $128 \mathrm{~KB}$ apart (and often land in the same cache set).

Horn's [71] breaks virtual machine isolation using a heuristic to find small eviction sets by iterating over Test 4.3 several times, and discarding all elements that are always hot (i.e. always produce cache hits). While this heuristic performs extremely well in practice, its asymptotic cost is quadratic on the set size.

Finally, a more recent work on cache attacks from portable code [76] $(\mathrm{PNaCl}$ and WebAssembly) discusses the problem of finding eviction sets on regular $4 \mathrm{~KB}$ pages and how to partially deal with TLB thrashing.

In contrast to these approaches, our work is the first to consider adversaries with less than 12 bits of control over the physical addresses, it formalizes the problem of finding eviction sets, and provides new techniques that might enable purely dynamic approaches.

Reverse engineering of slicing functions Modern CPUs ${ }^{5}$ with LLC slicing use proprietary hash functions for distributing blocks into slices, which lead to attempts to reverse engineer them. These works are based on: 1) allocating and identifying sets of colliding addresses [85, 77]; and 2) reconstructing the slice function using the hamming distance [86], or solving systems of equations [87], between these addresses. Even though we now know the slice hash function for several microarchitectures, and Maurice et al. [78] leverage it to speed up the finding of eviction sets with huge pages, we believe that its use on real attacks is hindered by constrained environments with scarce information about the physical addresses.

Thrashing/scanning resistant replacement policies Modern replacement policies such as insertion policies [88] or DRRIP [24], are known to perform better than PLRU against workloads causing scanning or thrashing. However, they also make eviction less reliable, and fall outside our current models (see Section 4.1). Howg [25] proposes a dual pointer chasing to mitigate these effects;

\footnotetext{
${ }^{5}$ According to Intel's Architecture Reference Manual [2] (see 2.4.5.3 Ring Interconnect and Last Level Cache), Sandy Bridge is the first generation with slicing.
} 
and Gruss et al. [66] generalize the approach with eviction strategies, which are access patterns over eviction sets that increase the chance of eviction under some unknown modern policies. Both approaches are orthogonal to our in that they already assume the possession of eviction sets.

Set index randomization Concurrent work proposes some new designs for randomized caches [89, 90], where cache sets are indexed with a keyed function that completely voids any attacker control over the physical address bits. A key result of these proposals is that they make cache-based attacks, and specially finding small eviction sets, more difficult. Their security analysis, however, considers quadratic attackers; it will be interesting to see how it is affected by our linear-time algorithm. 


\section{Learning Replacement Policies from Hardware Caches}

Understanding the timing behavior of modern CPUs is crucial for optimizing code and for ensuring timing-related security and safety properties. Examples of such properties are bounds on programs' worst-case execution time [91] or on the amount of information leaked via timing [92, 93]. Unfortunately, the timing behavior of today's high-performance processors depends on subtle and poorly documented details of their microarchitecture, which has triggered laborious efforts to build models of different components [94, 3, 4, 95].

Cache replacement policies have received specific attention [96, 97, 98, 25, 99], because they control the content of the memory hierarchy and hence heavily influence execution time. Detailed policy models are used in worst-case execution time analyzers [100], CPU simulators [101], and for improving microarchitectural attacks and defenses [98, 92, 93].

However, only few authors have approached the problem of inferring replacement policies in a principled way.

- Rueda [96] uses off-the-shelf techniques for learning register automata to infer cache replacement policies. The approach learns replacement policies with small state-spaces from noiseless simulator traces, but it has not been successfully applied to actual hardware.

- Abel and Reineke [97] present an approach that infers so-called permutationbased replacement policies, which include LRU, FIFO, and PLRU [102]. The approach has been used to infer policies from hardware performance counter measurements on hardware. However, permutation-based policies are restrictive in that they do not include important examples such as MRU [103], SRRIP [24], or the policies implemented in the lower-level caches of recent Intel CPUs.

Furthermore, both approaches share a drawback: the inferred policies are not easily interpretable by humans. 


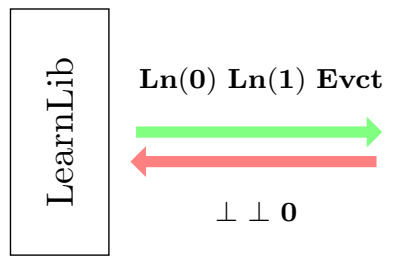

(a) LearnLib issues membership queries to the system under learning (SUL). The salient feature of our approach is that the language of the SUL refers to cache lines and not to the cache content. When the learning loop terminates, our tool returns an automaton describing the cache replacement policy under learning.
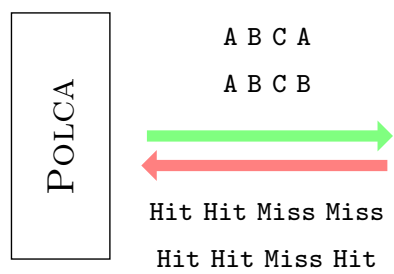

(b) PolcA translates a sequence of requests for cache lines $\operatorname{Ln}(\mathbf{i})$ or evictions Evct into sequences of abstract memory blocks. For this, the algorithm keeps track of the current cache state (here: blocks A/B in lines 0/1). Evct spawns multiple sequences that first produce a cache miss (here: C), followed by accesses to all previously contained blocks to infer which line was evicted (here: $\mathbf{0}$ ).
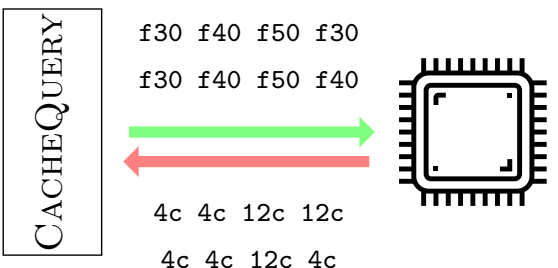

(c) CacheQuery receives as input sequences of abstract blocks (e.g., A B C A) and translates them into distinct concrete memory blocks (e.g., $A->f 30)$ that all map into the same cache set. It loads the corresponding memory blocks, counts the corresponding clock cycles, and returns for each load whether it was a cache hit (e.g., 4c->Hit) or a miss.

Figure 5.1: Leveraging POLCA and CACHEQUeRY to learn a toy replacement policy of a 2-way set associative CPU cache using the LearnLib [1] framework.

Approach. In this paper we propose an approach for learning cache replacement policies that goes beyond the state-of-the-art in that it (1) can learn arbitrary deterministic policies (2) from real-time (or performance counter) measurements on silicon CPUs. Moreover, we show how to (3) apply program synthesis to yield human-readable interpretations of the inferred policies.

Our approach relies on two contributions that enable us to leverage off-theshelf automata learning tools $[104,1]$ for attacking the problem:

- A tool, called CACHEQUERY, that provides an abstract interface to any individual cache set within the cache hierarchy ${ }^{1}$ of a silicon CPU. With CACHEQUERY, users can specify a cache set (say: set 63 in the L2 cache) and a pattern of memory accesses (say: A B C A B C), and they receive as output a sequence (say: Miss Miss Miss Hit Hit Hit) representing the hits and misses produced when performing a sequence of memory loads to addresses that are mapped into the specified cache set and that follow the specified pattern. CACHEQUERY liberates the user from dealing with intricate details such as the virtual-to-physical memory mapping, cache slicing, set indexing, interferences from other levels of the cache hierarchy, and measurement noise, and thus enables civilized interactions with an individual cache set. See Figure 5.1c.

\footnotetext{
${ }^{1}$ For a primer on hardware caches, see Section 2.2.
} 
- An algorithm, called POLCA, that provides an abstract interface to the cache replacement policy based on an interface to a cache set, such as CACHEQUeRY. PolcA translates inputs to the replacement policy (which refer to the cache lines) into inputs to the cache set (which refer to the memory blocks that are stored in it). To achieve this, PoLCA itself keeps track of the current cache content, e.g., by issuing queries to the cache interface to determine which block has been evicted in a miss. POLCA exploits the data-independence symmetry of the replacement policy that would otherwise have to be inferred by the learning algorithm, and it is key to making automata learning work in this domain. See Figure 5.1b.

We use PoLCA as a so-called membership oracle for the replacement policy to be learned from an abstract cache set (which can be implemented by CACHEQUERY or by a software-simulated cache). The oracle provides an interface to libraries such LearnLib [1], which enables us to leverage the state-of-the-art in automata learning for inferring replacement policies of silicon CPUs. We give formal relative completeness guarantees for the learned policies, based on the correctness of POLCA and LearnLib. The full learning pipeline is depicted in Figure 5.1.

Finally, we show how to use program synthesis to automatically derive a higher-level representation of the learned replacement policy. The main component of our synthesis step is a program template for replacement policies, which we base on concepts used to describe replacement policies in the microarchitecture community [24]. By combining the policy template with a set of constraints derived from the learned automaton, we can rely on off-the-shelf synthesis solvers to synthesize high-level policy representations [105].

Evaluation. We evaluate our approach in 3 case studies:

1. We evaluate the scalability of learning replacement policies with POLCA. To this end, we learn a comprehensive set of deterministic policies (including FIFO, LRU, PLRU [102], MRU [103], LIP [106], and different variants of SRRIP [24]) from the noiseless hit-miss traces produced by a software-simulated cache. Our experiments demonstrate that POLCA enables LearnLib to infer policies with state-spaces of more than 2 orders of magnitude larger than what was reported for direct applications of LearnLib to simulator traces [96].

2. We evaluate the effectiveness of learning with PolcA and CACHEQuery on the L1, L2, and L3 caches of three recent Intel CPUs. Our experiments show that we can effectively learn cache replacement policies up to associativity 8 from timing measurements on modern CPUs. While some of the policies were known, we do uncover 2 policies that have not yet been documented in the literature.

3. We evaluate our template-based synthesis approach by synthesizing programs for 8 out of 9 different policies (obtained from both the simulators and silicon CPUs), for a fixed associativity 4. This allows us to provide high-level descriptions for the 2 previously undocumented policies. 
Summary of Contributions. In summary, we present a practical end-to-end solution for inferring deterministic cache replacement policies using off-the-shelf techniques for automata learning and program synthesis. The enabling contribution is a chain of two abstractions that exposes a membership oracle to the cache replacement policy, based on timing measurements on a silicon CPU.

Our tools, CACHEQUERY and POLCA, are available, together with the learned models and synthesized programs, at https://github.com/cgvwzq/cachequery/ and https://github.com/cgvwzq/polca/ respectively.

\subsection{Modeling Caches and Policies}

In this section we present our model of hardware caches. A key feature, inspired by [107], is that we distinguish between the replacement policy (Section 5.1.1), which determines the cache lines to be replaced, and the cache itself (Section 5.1.2), which stores and retrieves memory blocks (according to the replacement policy).

In this work, we consider $n$-way set associative caches, i.e., caches where all cache sets consist of $n$ lines, and we focus on individual cache sets. For brevity's sake, in the following we refer to a set of an $n$-way cache simply as an $n$-way cache.

\subsubsection{Replacement Policy Model}

We model the replacement policy of a cache set as a deterministic, finite-state Mealy machine that accepts inputs of the form $\operatorname{Ln}(\mathbf{i})$, for accessing the $i$-th cache line, and Evct, for requesting a cache line to be freed (see Table 5.1). Given an input, the policy updates its control state and outputs the index of the line to be freed (or $\perp$ otherwise).

Table 5.1: Policy and cache alphabets (associativity $n$ )

\begin{tabular}{l|cc} 
& Policy & Cache \\
\hline Input & $\{\mathbf{L n}(\mathbf{0}), \ldots, \mathbf{L n}(\mathbf{n}-\mathbf{1})\} \cup\{$ Evct $\}$ & Blocks \\
Output & $\{\perp\} \cup\{\mathbf{0}, \ldots, \mathbf{n}-\mathbf{1}\}$ & $\{$ Hit, Miss $\}$
\end{tabular}

Definition 2. A replacement policy of associativity $n \in \mathbb{N}$ is a Mealy machine $\left\langle\mathbf{C S}, \mathbf{c s}_{\mathbf{0}}, \mathbf{I P}, \mathrm{OP}, \delta, \lambda\right\rangle$ consisting of:

- a finite set of control states $\mathbf{C S}$;

- an initial control state $\mathbf{c s}_{\mathbf{0}} \in \mathbf{C S}$;

- the set of inputs $\mathbf{I P}=\{\mathbf{L n}(\mathbf{0}), \ldots, \mathbf{L n}(\mathbf{n}-\mathbf{1})\} \cup\{$ Evct $\}$;

- the set of outputs $\mathrm{OP}=\{\perp\} \cup\{\mathbf{0}, \ldots, \mathbf{n}-\mathbf{1}\}$;

- a transition function $\delta: \mathbf{C S} \times \mathbf{I P} \rightarrow \mathbf{C S}$; and 
- an output function $\lambda$ : $\mathbf{C S} \times \mathbf{I P} \rightarrow \mathbf{O P}$.

We require that, (a) $\lambda$ returns a value in $\{\mathbf{0}, \ldots, \mathbf{n}-\mathbf{1}\}$ when given the input Evct; and (b) $\lambda$ returns $\perp$ when given an input in $\{\operatorname{Ln}(\mathbf{0}), \ldots, \operatorname{Ln}(\mathbf{n}-\mathbf{1})\}$.

We write $\mathbf{c s} \stackrel{\langle\mathbf{i}, \mathbf{o}\rangle}{\longrightarrow} \mathbf{c s}^{\prime}$ when $\delta(\mathbf{c s}, \mathbf{i})=\mathbf{c s}^{\prime}$ and $\lambda(\mathbf{c s}, \mathbf{i})=\mathbf{o}$.

We now introduce the trace semantics of policies, where traces are sequences of input/output pairs. We use standard sequence notation: $S^{*}$ is the set of finite sequences over $S, \varepsilon$ is the empty sequence, and $s_{1} \cdot s_{2}$ denotes sequence concatenation. is the set $\llbracket \mathbf{P} \rrbracket \subseteq(\mathbf{I P} \times \mathbf{O P})^{*}$ of all sequences $\left\langle\mathbf{i}_{1}, \mathbf{o}_{\mathbf{1}}\right\rangle \cdot\left\langle\mathbf{i}_{\mathbf{2}}, \mathbf{o}_{\mathbf{2}}\right\rangle \cdot \ldots \cdot\left\langle\mathbf{i}_{\mathbf{m}}, \mathbf{o}_{\mathbf{m}}\right\rangle$ for which there are control states $\mathbf{c s}_{\mathbf{1}}, \ldots, \mathbf{c s}_{\mathbf{m}}$ such that $\mathbf{c s}_{\mathbf{0}} \stackrel{\left\langle\mathbf{i}_{1}, \mathbf{o}_{1}\right\rangle}{\longrightarrow} \mathbf{c s}_{\mathbf{1}} \stackrel{\left\langle\mathbf{i}_{2}, \mathbf{o}_{2}\right\rangle}{\longrightarrow} \ldots \stackrel{\left\langle\mathbf{i}_{\mathbf{m}}, \mathbf{o}_{\mathbf{m}}\right\rangle}{\longrightarrow} \mathbf{c s}_{\mathbf{m}}$.

Example 2. Consider a Least Recently Used (LRU) replacement policy, where the least recently used cache line is the one to be evicted.

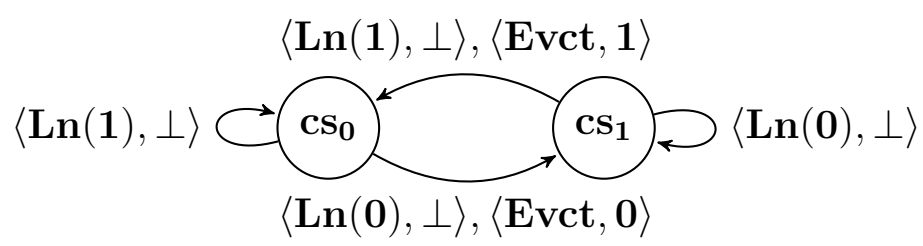

There are two control states $\mathbf{c s}_{\mathbf{0}}$ and $\mathbf{c s}_{\mathbf{1}}$, where $\mathbf{c s}_{\mathbf{i}}$ indicates that line $\mathbf{i}$ is next to be evicted (i.e., $\mathbf{i}$ is the line storing the least recently used memory block).

\subsubsection{Cache Model}

We model a cache of associativity $n$ as a Labeled Transition System (LTS) that accepts as input elements $b$ from a potentially infinite set of memory blocks Blocks, and that produces as output a Hit when block $\mathrm{b}$ is in the cache, and a Miss otherwise (see Table 5.1).

Each state of the cache is a pair $\langle\mathbf{c c}, \mathbf{c s}\rangle$ consisting of the cache content $\mathrm{cc} \in$ $\mathrm{CC}^{\mathrm{n}}$, which is an $n$-tuple of memory blocks without repetitions, and the control state cs of a replacement policy $\mathbf{P}$. Formally:

Definition 3. An $n$-way cache induced by a policy $\mathbf{P}=\left\langle\mathbf{C S}, \mathbf{c s}_{\mathbf{0}}, \mathbf{I P}, \mathbf{O P}, \delta, \lambda\right\rangle$ is an LTS $\mathrm{C}\left(\mathbf{P}, \mathrm{cc}_{0}, n\right)=\left\langle\mathrm{S}, \mathrm{s}_{0}, \mathrm{IC}, \mathrm{OC}, \Longrightarrow\right\rangle$ consisting of:

- a set of cache states $\mathrm{S}=\mathrm{CC}^{\mathrm{n}} \times \mathbf{C S}$;

- an initial cache state $\mathbf{s}_{0}=\left\langle\mathrm{cc}_{0}, \mathbf{c s}_{\mathbf{0}}\right\rangle \in \mathrm{S}$;

- a set of inputs IC = Blocks;

- a set of outputs $\mathrm{OC}=\{$ Hit, Miss $\}$;

- a transition relation $\Longrightarrow \subseteq \mathrm{S} \times \mathrm{IC} \times \mathrm{OC} \times \mathrm{S}$ that is induced by the policy $\mathbf{P}$ following Figure 5.2.

The cache's transition relation relies on two rules, see Fig. 5.2: 


$$
\frac{\mathrm{cc}[\mathbf{i}]=\mathrm{b} \quad \operatorname{cs} \stackrel{\langle\mathbf{L n}(\mathbf{i}), \perp\rangle}{\longrightarrow} \mathbf{c s}^{\prime}}{\langle\mathrm{cc}, \mathrm{cs}\rangle \stackrel{\langle\mathrm{b}, \mathrm{Hit}\rangle}{\Longrightarrow}\left\langle\mathrm{cc}, \mathbf{c s}^{\prime}\right\rangle} \mathrm{HIT}
$$

$$
\frac{\forall \text { i. } c c[i] \neq b \quad \quad c s \stackrel{\langle\text { Evct }, \mathbf{i}\rangle}{\longrightarrow} \mathrm{cs}^{\prime}}{\langle\mathrm{cc}, \mathrm{cs}\rangle \stackrel{\langle\mathrm{b}, \text { Miss }\rangle}{\longrightarrow}\left\langle\mathrm{cc}[\mathbf{i} \mapsto \mathrm{b}], \mathrm{cs}^{\prime}\right\rangle} \text { Miss }
$$

Figure 5.2: Transition relation for a cache $\Longrightarrow$ given that of a replacement policy $\rightarrow$. Here, cc $[\mathbf{i}]$ denotes the block stored in cc's $\mathbf{i}$-th line and cc $[\mathbf{i} \mapsto b]$ the cache content obtained by replacing the block in the $\mathbf{i}$-th line with $\mathrm{b}$.

The rule HiT captures what happens upon access to a block that is cached: The rule (1) determines that $\mathrm{b}$ is stored in cc's $\mathbf{i}$-th line, and (2) updates the control state by executing the policy with input $\operatorname{Ln}(\mathbf{i})$.

The rule Miss captures what happens upon access to a block that is not cached: The rule (1) checks that the block $\mathrm{b}$ is not in the cache, (2) determines the line $\mathbf{i}$ of the block to evict by executing the policy with input Evct, and (3) inserts $\mathrm{b}$ in the $\mathbf{i}$-th line and updates the cache state.

Note that the cache's transition relation directly updates only the cache content cc. Changes to the control state cs are mediated by the replacement policy, which takes as input only accesses to cache lines $\operatorname{Ln}(\mathbf{i})$ or eviction requests Evct. Hence, updates to the control state are agnostic to the accessed memory blocks.

Similarly to a policy's semantics, we introduce a cache's semantics. The trace semantics of a cache $\mathrm{C}$ (short: cache semantics) is the set $\llbracket \mathrm{C} \rrbracket \subseteq(\mathrm{IC} \times \mathrm{OC})^{*}$ of all sequences $\left\langle i_{1}, o_{1}\right\rangle \cdot\left\langle i_{2}, o_{2}\right\rangle \cdot \ldots \cdot\left\langle i_{m}, o_{m}\right\rangle$ for which there are cache states $s_{1}, \ldots, s_{m}$ such that $\mathbf{s}_{0} \stackrel{\left\langle i_{1}, o_{1}\right\rangle}{\Longrightarrow} s_{1} \stackrel{\left\langle i_{2}, o_{2}\right\rangle}{\Longrightarrow} \ldots \stackrel{\left\langle i_{m}, o_{m}\right\rangle}{\Longrightarrow} s_{m}$.

Example 3. The LTS of the cache induced by the LRU policy from Example 2 is as follows (we depict only part of the infinite LTS for 3 abstract blocks A, B, and $\mathrm{C}$ ):

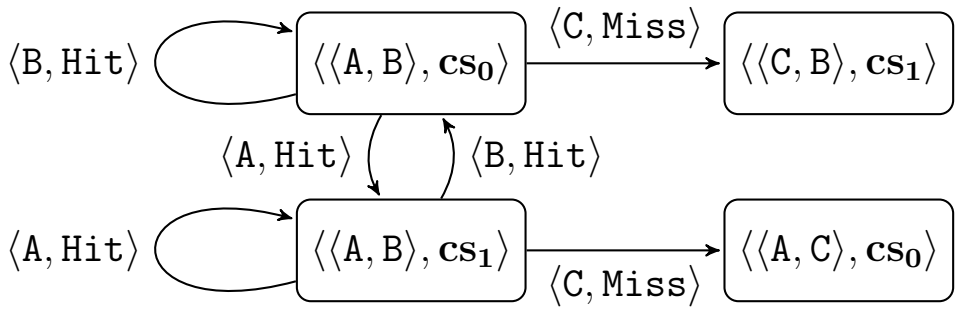

Consider the cache state $\left\langle\langle A, B\rangle, \mathbf{c s}_{\mathbf{0}}\right\rangle$. Accessing the block B produces a Hit since $\mathrm{B}$ is stored in line 1 . Hence, we modify neither the cache content nor the control state because $\mathbf{c s}_{\mathbf{0}} \stackrel{\langle\mathbf{L n}(\mathbf{1}), \perp\rangle}{\longrightarrow} \mathbf{c s}_{\mathbf{0}}$ according to the policy. Accessing the block A, which is stored in line 0 , also produces a Hit. This time, however, we update the control state since the least recently used cache line is now 1, i.e., $\mathbf{c s}_{\mathbf{0}} \stackrel{\langle\mathbf{L n}(\mathbf{0}), \perp\rangle}{\longrightarrow} \mathbf{c s}_{\mathbf{1}}$. 
In contrast, accessing the block $\mathrm{C}$, which is not in the cache, leads to a Miss. The replacement policy determines that the block $\mathrm{C}$ has to be stored in line 0, i.e., $\mathbf{c s}_{\mathbf{0}} \stackrel{\langle\text { Evct, } \mathbf{0}\rangle}{\longrightarrow} \mathbf{c s}_{\mathbf{1}}$, and the new cache state is $\left\langle\langle\mathrm{C}, \mathrm{B}\rangle, \mathbf{c s}_{\mathbf{1}}\right\rangle$.

\subsection{Polca: Learning Replacement Policies}

In this section, we present our policy learning approach. We begin by introducing background on automata learning (Section 5.2.1). Next, we describe the two main components of our learning approach, namely oracles for membership (Section 5.2.2) and equivalence queries (Section 5.2.3) for replacement policies. Finally, we describe our prototype implementation of POLCA on top of LearnLib (Section 5.2.4).

\subsubsection{A Primer on Automata Learning}

The prevalent approach to learning automata follows the student-teacher paradigm established by Angluin [108] and extended to Mealy machines by Niese [109]. There, the student's goal is to learn an unknown Mealy machine $M$ by asking queries to the teacher. There are two types of queries:

1. membership queries, where the student asks whether a given trace belongs to the machine $M$, and

2. equivalence queries, where the student asks whether a hypothesized Mealy machine $H$ is (trace) equivalent to $M$.

Initially, the student knows only the input and output alphabets. By making a finite number of queries to the teacher as prescribed by the learning algorithm, the student eventually learns $M$.

We next show how to build oracles to answer membership and equivalence queries for a replacement policy, based on interactions with a hardware cache, which enables us to leverage automata learning algorithms for inferring replacement policies.

\subsubsection{Membership Queries}

We now present PoLCA, an algorithm that provides a membership oracle for a replacement policy, given a cache that implements that policy. That is, PoLCA takes as input a trace $\mathbf{t}$ of policy inputs and outputs, a cache $\mathbf{C}$ induced by a policy $\mathbf{P}$, and it determines whether $\mathbf{t} \in \llbracket \mathbf{P} \rrbracket$. For that, PoLCA translates $\mathbf{t}$ into a series of input traces (i.e., sequences of memory blocks) to the underlying cache $\mathrm{C}$, by internally keeping track of the blocks stored in the cache. From the outputs of $\mathrm{C}$, PolcA then deduces whether $\mathbf{t} \in \llbracket \mathbf{P} \rrbracket$.

By extracting the policy semantics $\llbracket \mathbf{P} \rrbracket$ from the cache semantics $\llbracket C \rrbracket$, PoLCA effectively inverts the transition rules in Figure 5.2. This enables learning only 
the policy $\mathbf{P}$, rather than learning also the data storage logic of the cache $\mathbf{C}$, and is key for scaling automata learning to hardware caches.

Algorithm. The pseudocode of PoLCA is given as Algorithm 3. It receives as input an initial cache content $\mathrm{cc}_{0}$, a policy trace $\mathbf{t} \in(\mathbf{I P} \times \mathbf{O P})^{*}$, and the cache semantics $\llbracket C \rrbracket$; and it outputs true if $\mathbf{t}$ belongs to the policy semantics $\llbracket \mathbf{P} \rrbracket$, and false otherwise.

POLCA relies on the following helper functions:

- probeCache which, given a trace of blocks $\mathrm{q}$ and the cache semantics $\llbracket \mathrm{C} \rrbracket$, accesses all blocks in $\mathrm{q}$ and returns whether the last block produces Hit or Miss according to the cache semantics $\llbracket C \rrbracket$.

- mapInput which, given a policy input ip and a cache content cc, maps ip to a memory block b. If ip is $\mathbf{L n}(\mathbf{i})$, the function returns cc $[i]$. Otherwise (i.e., ip is Evct), it returns a block b not in cc.

- mapOutput which, given a cache output oc, a trace of blocks q, a cache content cc, and the cache semantics $\llbracket C \rrbracket$, maps oc to the line containing the block that is evicted. If oc is Hit, the function returns $\perp$. Otherwise (i.e., oc is Miss), it returns the line $\mathbf{i}$ where the evicted block was stored.

- findEvicted which, given a trace of blocks q, a cache content cc, and the cache semantics $\llbracket C \rrbracket$, determines which line has been evicted by the last block in q. For that, the function probes the cache with block traces $q \cdot \mathrm{cc}[1], \ldots, \mathrm{q} \cdot \mathrm{cc}[n]$ and determines which block resulted in Miss, i.e., the line that has been evicted by the last block in $\mathrm{q}$.

We are now ready to describe PoLCA in detail: For each pair $\langle\mathbf{i p}, \mathbf{o p}\rangle \in \mathbf{t}$, the algorithm maps the input policy symbol ip to a memory block $\mathrm{b}$ (mapInput call at line 5). Then, the algorithm probes the cache to determine the result oc of accessing b. Next, the cache output oc is mapped to an output policy symbol op' ( map Output call at line 7). If op does not match the computed $\mathbf{o p}^{\prime}$, the algorithm returns false; else, the algorithm moves to the next pair of input/output policy symbols, and returns true when the sequence is entirely processed.

For this, POLCA keeps track of the sequence of blocks processed so far (through the ic variable, which is updated after every cache miss in line 9) and the sequence of blocks processed so far (through the ic variable, which is updated with every call to mapInput). ic is used to set the cache $\mathrm{C}$ into the correct state before accessing the new block ic $[i]$ (line 6), and for identifying the cache line that was last evicted (findEvicted function).

Correctness. Theorem 2 states that, given a cache $\mathbf{C}$ with unknown policy $\mathbf{P}$, PoLCA provides a sound, complete and terminating oracle for $\mathbf{P}$ 's trace semantics.

Theorem 2. Let $\mathrm{C}$ be a cache of associativity $n$ with initial content $\mathrm{Cc}_{0} \in \mathrm{CC}^{\mathrm{n}}$ and policy $\mathbf{P}$. Then, $\llbracket \mathbf{P} \rrbracket=\left\{\mathbf{t} \in(\mathbf{I P} \times \mathbf{O P})^{*} \mid \operatorname{PoLCA}\left(\mathrm{cc}_{0}, \mathbf{t}, \llbracket C \rrbracket\right)=\right.$ true $\}$. 


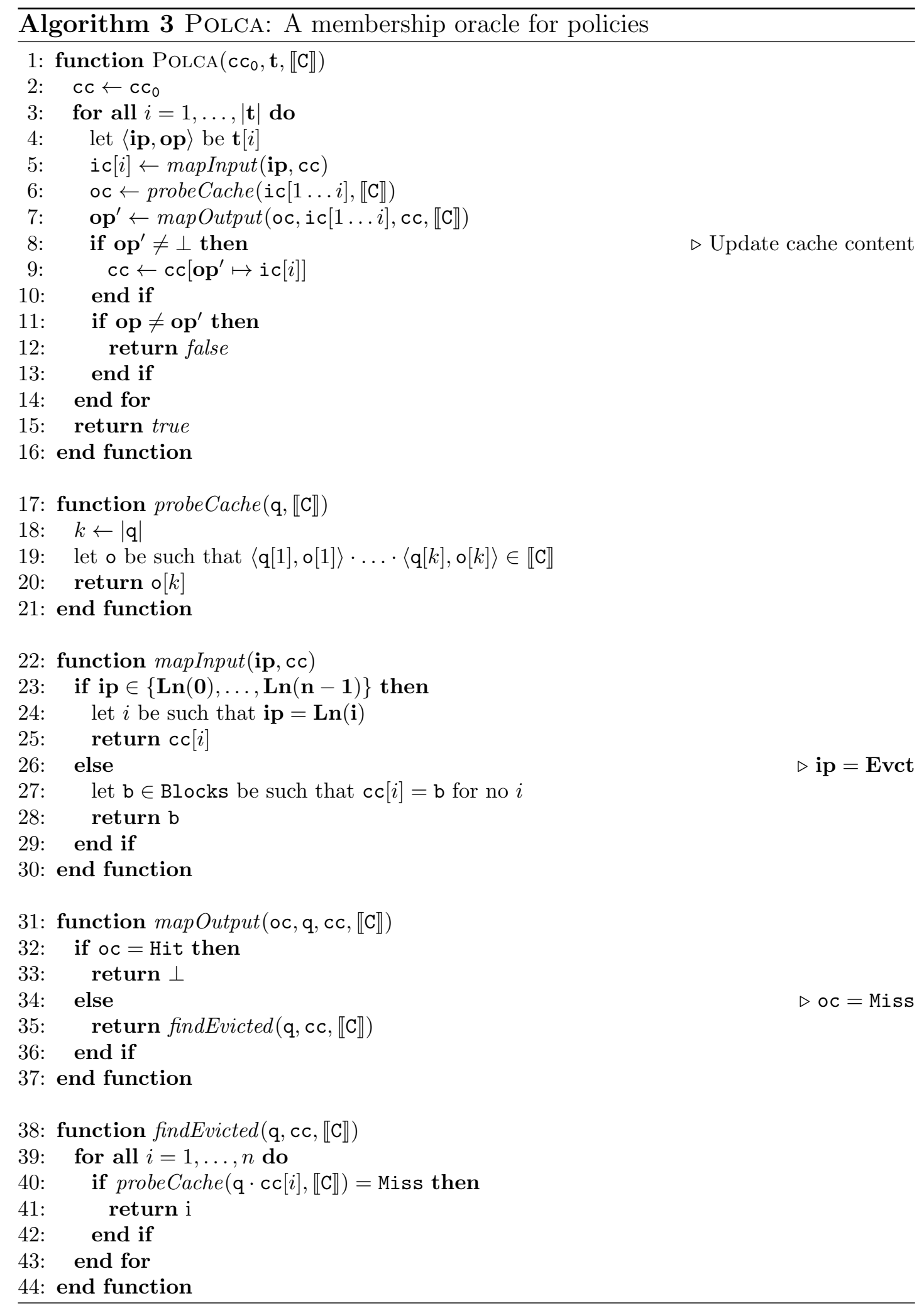


As a corollary to Theorem 2, we obtain a language-theoretic relationship between policies and caches. Proposition 5 states that, once we fix the initial cache content $\mathrm{cc}_{0}$ and associativity $n$, a replacement policy $\mathbf{P}$ uniquely determines the corresponding cache $\mathrm{C}\left(\mathbf{P}, \mathrm{cc}_{0}, n\right)$.

Proposition 5. Given two policies $\mathbf{P}$ and $\mathbf{P}^{\prime}$ of associativity $n \in \mathbb{N}$ and an initial cache content $\mathrm{cc}_{0} \in \mathrm{CC}^{\mathrm{n}}$, then $\llbracket \mathbf{P} \rrbracket=\llbracket \mathbf{P}^{\prime} \rrbracket$ iff $\llbracket \mathrm{C}\left(\mathbf{P}, \mathrm{cc}_{0}, n\right) \rrbracket=\llbracket \mathbf{C}\left(\mathbf{P}^{\prime}, \mathrm{cc}_{0}, n\right) \rrbracket$.

Concretely, Proposition 5 provides a theoretical justification for learning only the policy, since knowing the policy is equivalent to knowing the cache behavior.

\subsubsection{Equivalence Queries}

Equivalence queries between two Mealy machines $M$ and $M^{\prime}$ are commonly implemented using conformance testing, which relies on a test suite (TS) of membership queries: If there is a membership query in TS on which $M$ and $M^{\prime}$ disagree, the machines are clearly not equivalent. However, there are Mealy machines $M$ for which there is no finite test suite that demonstrates non-equivalence for all machines $M^{\prime}$ with $\llbracket M \rrbracket \neq \llbracket M^{\prime} \rrbracket$. That is, the approximation of equivalence queries using finite membership tests is not complete [110].

In our approach, we hence aim for a weaker notion of completeness due to [110]. Namely, for a parameter $m$ we say that a test suite TS is $m$-complete for a hypothesized policy $\mathbf{H}$, if there is no policy $\mathbf{P}$ with less than $m$ control states and $\llbracket \mathbf{H} \rrbracket \neq \llbracket \mathbf{P} \rrbracket$, such that both $\mathbf{H}$ and $\mathbf{P}$ agree on the TS. With this, one can obtain the following guarantees for the equivalence test.

Theorem 3. Let $\mathbf{P}$ be an unknown replacement policy, $\mathbf{H}$ a hypothesized policy, and TS an $m$-complete test suite for $\mathbf{H}$ for some $m \in \mathbb{N}$. If $\mathbf{P}$ and $\mathbf{H}$ agree on all queries of TS then either $\llbracket \mathbf{H} \rrbracket=\llbracket \mathbf{P} \rrbracket$ or $\mathbf{P}$ has more than $m$ states.

We use existing algorithms, e.g. the Wp-Method [111], to compute $m$-complete suites for our hypothesized policy $\mathbf{H}$.

\subsubsection{Tool Implementation}

We implement PoLCA in a Java prototype on top of the LearnLib automata framework v0.14.0 [1]. This allows us to leverage state-of-the-art automata learning algorithms.

To access the cache semantics $\llbracket C \rrbracket$, our tool interacts either with a softwaresimulated cache or with CACHEQUERY when targeting real hardware.

Concretely:

- for the membership oracle, we implement PoLCA, as described in Section 5.2.2. 
- for the equivalence oracle, we rely on the Wp-Method [111] for computing test suites for conformance testing, as described in Section 5.2.3. Ideally, one would use a $m$-complete TS for $\mathbf{H}$, for $m$ as large as possible. Unfortunately the cost of computing $m$-complete test suite grows exponentially with $m$. To achieve a good trade-off between completeness guarantees and complexity, we rely on $(|\mathbf{H}|+k)$-complete TS, for a small constant $k$ which we call the depth of the suite.

Our main learning loop uses the $k$-deep conformance test for finding counterexamples. If the test fails, i.e., a counterexample for the current hypothesis is found, we refine the hypothesis and learning continues. Otherwise, the learning terminates and we output the current hypothesis. At the end, we get the following overall guarantees.

Corollary 4. If our learning approach with PoLCA, applied to a cache $\mathrm{C}\left(\mathbf{P}, \mathrm{cc}_{0}, n\right)$, returns a policy $\mathbf{P}^{\prime}$, then $\llbracket \mathbf{P} \rrbracket=\llbracket \mathbf{P}^{\prime} \rrbracket$, or $\mathbf{P}$ has more than $\left|\mathbf{P}^{\prime}\right|+k$ states.

To avoid cumbersome notation, from this point on we use POLCA to refer to both our algorithm and to our prototype tool integrating the algorithm with the learning loop.

\subsection{CacheQuery: An Interface to Hardware Mem- ory Caches}

In this section we present CACHEQUERY, a tool for querying silicon CPU caches that frees the user from low-level details like slicing, mapping, virtual-to-physical translation, and timing.

We first describe MemBlockLang, the domain-specific language for specifying inputs to CACHEQUERY; then we describe CACHEQUERY's architecture and discuss some of the implementation challenges.

\subsubsection{Domain Specific Language}

We design MemBlockLang (MBL), a macro language that facilitates the writing of queries to caches.

A query is a sequence of one or more memory operations. Each memory operation is specified as a block from a finite, ordered set of blocks Blocks, and it is decorated with an optional tag from $\{?, !\}$. The tag '?' indicates that the access to the block should be profiled [112] to determine whether it results in a cache hit or miss; the tag '!' indicates that the block is invalidated (e.g., via clflush); and no tag means that the block is just accessed.

MBL features several macros that facilitate writing common query sequences:

- An expansion macro '@' that produces a sequence of associativity many different blocks in increasing order, starting from the first element. For example, for associativity 8, @ expands to the sequence of blocks A B C D E F G H. 


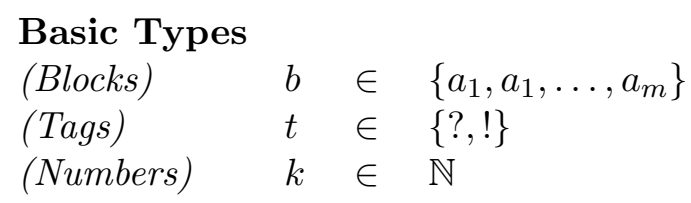

\section{Syntax}

$$
\begin{array}{rlll}
\text { (Queries) } & q:= & \varepsilon|b|(b) t \mid q_{1} \cdot q_{2} \\
\text { (Expressions) } & s:= & (q)\left|\left\{q_{1}, \ldots, q_{l}\right\}\right| @|-|(s) t \mid \\
& s_{1} \circ s_{2}\left|\left(s_{1}\right)\left[s_{2}\right]\right|(s) k
\end{array}
$$

Figure 5.3: MBL syntax. We assume a given ordered set of blocks $\mathbb{B}=$ $\left\{a_{1}, a_{1}, \ldots, a_{m}\right\}$ and a value $n \in \mathbb{N}$ representing the cache's associativity such that $n<m$.

- A wildcard macro '_' produces associativity many different queries, each one consisting of a different block. As for ' $₫$ ', blocks are chosen in alphabetical order. For example, for associativity 8, expands to the set of queries $\{A$, B, C, D, E, F, G, H\}.

- A concatenation macro $s_{1} \circ s_{2}$ that concatenates each query in $s_{1}$ 's expansion with each query in $s_{2}$ 's expansion. For instance, (A B C D) $\circ(E F)$ expands to the query A B C D E F.

- An extension macro $s_{1}\left[s_{2}\right]$ that takes as input queries $s_{1}$ and $s_{2}$ and then creates $\left|s_{2}\right|$ many copies of $s_{1}$ and extends each of them with a different element of $s_{2}$. For example (A B C D) [E F] expands to the set of queries $\left\{\begin{array}{l}A \\ B\end{array}\right.$ C D E, A B C D F $\}$.

- A power operator $(s) n$ that repeats a query macro $s$ for $n$ times. For example, (A B C) 3 expands to the query A B C A B C A B C.

- A tag over $\left(s_{1}\right)$ or $\left[s_{1}\right]$ applies to every block in $s_{1}$. That is, (A B)? expands to $\mathrm{A}$ ? $\mathrm{B}$ ?.

Figure 5.3 shows how MBL expressions can be given a formal semantics in terms of sets of queries.

Semantics. The semantics of an MBL expression $s$ consists of a set of queries $\llbracket s \rrbracket$ and it is defined as follows:

- $\llbracket q \rrbracket=\{q\}$ where $q$ is a query.

- $\llbracket\left\{q_{1}, \ldots, q_{l}\right\} \rrbracket=\left\{q_{1}, \ldots, q_{l}\right\}$ where $\left\{q_{1}, \ldots, q_{l}\right\}$ is a set of queries.

- $\llbracket \mathbb{Q} \rrbracket=\left\{a_{1} \cdot a_{2} \cdot \ldots \cdot a_{n}\right\}$.

- $\llbracket \rrbracket=\left\{a_{1}, a_{2}, \ldots, a_{n}\right\}$. 
- $\llbracket s ? \rrbracket=\left\{a_{1} ? \cdot a_{2} ? \cdot \ldots \cdot a_{k} ? \mid a_{1} \cdot a_{2} \cdot \ldots \cdot a_{k} \in \llbracket s \rrbracket\right\}$ where $s$ is an MBL expression such that all queries in $\llbracket s \rrbracket$ do not have tags.

- $\llbracket s ! \rrbracket=\left\{a_{1} ! \cdot a_{2} ! \cdot \ldots \cdot a_{k} ! \mid a_{1} \cdot a_{2} \cdot \ldots \cdot a_{k} \in \llbracket s \rrbracket\right\}$ where $s$ is an MBL expression such that all queries in $\llbracket s \rrbracket$ do not have tags.

- $\llbracket s_{1} \circ s_{2} \rrbracket=\left\{s \cdot s^{\prime} \mid s \in \llbracket s_{1} \rrbracket \wedge s^{\prime} \in \llbracket s_{2} \rrbracket\right\}$ where $s_{1}, s_{2}$ are MBL expressions.

- $\llbracket s_{1}\left[s_{2}\right] \rrbracket=\left\{s \cdot a \mid s \in \llbracket s_{1} \rrbracket \wedge \exists s_{2} \in \llbracket s_{2} \rrbracket, i \in \mathbb{N} . s_{2}[i]=a\right\}$ where $s_{1}, s_{2}$ are MBL expressions.

- $\llbracket(s) k \rrbracket=\left\{s_{1} \cdot s_{2} \cdot \ldots \cdot s_{k} \mid \bigwedge_{1 \leq i \leq k} s_{i} \in \llbracket s \rrbracket\right\}$, where $s$ is an MBL expression and $k \in \mathbb{N}$.

- $\llbracket(s) \rrbracket=\llbracket s \rrbracket$, where $s$ is an MBL expression.

For the purpose of presentation, we focus on an example.

Example 4. For associativity 4, the query ' $\subseteq$ X _?' expands to ' $(A B C D) \circ X \circ$ [A B C D] ?' or, equivalently, to the set of queries $\{\mathrm{A} B \mathrm{C} D \mathrm{X} A$ ?, $\mathrm{A} B \mathrm{C} \mathrm{D} X \mathrm{~B}$ ?, A B C D X C?, A B C D X D?\}. This query performs an initial insertion (i.e., fills the cache with blocks A B C D), accesses a block X not in the cache, and probes all blocks A, B, C, and D to determine which one has been replaced after the cache miss caused by $\mathrm{X}$. In fact, this query can implement the function findEvicted in Algorithm 3.

\subsubsection{Architecture}

CacheQuery is split into two parts, described next. The backend is implemented in $\mathrm{C}$ as a Linux Kernel Module, while the frontend is implemented in Python 3.

Frontend. CACHEQUerY's frontend expands MBL expressions into sets of queries. The frontend provides two different execution modes: interactive and batch.

- The interactive mode provides a REPL shell for executing queries, modifying configuration options, and dynamically choosing the target cache level and set. We use this mode as an interface for the learning algorithm

- The batch mode allows to run groups of predefined queries against different cache sets. This becomes useful for running batteries of tests, which, for instance, allows us to identify fixed leader sets.

Furthermore, the frontend can use LevelDB ${ }^{2}$ to cache query responses. This improves performance by avoiding emitting repeated queries to the backend.

\footnotetext{
${ }^{2}$ LevelDB is a fast string key-value storage library: https://github.com/google/leveldb.
} 
Backend. CACHEQUERY's backend translates arbitrary queries into sequences of memory accesses, generates the appropriate machine code with the corresponding profiling, executes the code in a low-noise environment, and finally returns traces of hits and misses. We remark that profiling happens at the granularity of individual memory accesses, and it supports performance counters, time stamp counter, and counting core cycles, as demanded by the user

The backend is implemented as a Loadable Kernel Module (LKM). Which allows us to use APIs that provide fine-grained control over operating system details like virtual to physical address translation, interrupts, and preemption.

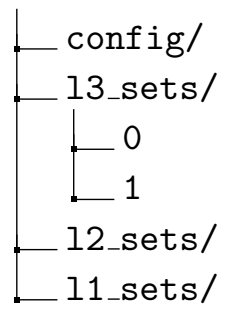

Figure 5.4: LKM's virtual file system used by CACHEQUERY

On load, the LKM allocates several pools of memory (one per cache level) and maps each memory block into its corresponding cache sets, one per cache level. This facilitates the address selection during code generation.

The backend provides a virtual file system interface, depicted in Figure 5.4, and all the communication is handled through read and write operations over virtual files. Specifically, writing query sequences into the cache set virtual file triggers the address selection and code generation, whereas reading from the cache set virtual file executes the generated code, and returns the sequence of hits and misses.

\subsubsection{Implementation Challenges}

In the following we discuss some of the challenges in implementing CACHEQUERY.

Set Mapping. The first challenge is identifying which memory addresses are mapped into which cache sets, i.e. which addresses are congruent. For this, we need to know the number of cache sets, if these sets are virtually or physically indexed, and how the mapping is performed, i.e., which bits of the address are used for the mapping. For most architectures, this information is publicly available [3, 4]. Otherwise, it is possible to infer it [97], or to dynamically find congruent addresses, as explained in Chapter 4. CACHEQUERY is completely parametric on the set mapping details, which need only to be determined once per microarchitecture.

Cache Filtering. When running queries against a low-level cache, say L3, one needs to make sure that the corresponding memory accesses do not hit higher-level caches 
such as L1. To this end, CACHEQUERY automatically evicts every accessed block from higher-level caches. For instance, after accessing a block b in L3, CACHEQUERY automatically accesses non-interfering eviction sets (i.e. addresses that are congruent with b in L2 and L1, but not congruent in L3) to ensure b's eviction from L2 and L1.

Code Generation. MBL expressions are first expanded into sets of queries, which are then dynamically translated into native code for execution. Each query is implemented as a function returning a 64-bit mask with the hit/miss pattern of the profiled memory blocks.

The generated code includes the necessary profiling instructions (e.g., rdtsc), the conditional moves to update the output bit mask, and the additional memory loads to evict higher cache levels. To support arbitrary queries, we use immediate load operations $^{3}$ serialized with memory fences, rather than the more common pointer chasing technique [113].

Interferences. To minimize noise during memory interactions, CACHEQUERY temporarily disables hardware prefetchers, hyper-threading, frequency scaling, and other cores. To minimize cache interferences, we repeatedly allocate the generated code until it does not conflict with the target cache set. Furthermore, the generated code is executed multiple times to reduce measurement noise.

\subsubsection{Limitations}

Currently, CACHEQUeRY only supports data caches (not instruction caches) in Intel CPUs. While several parts of our implementation are architecture-agnostic, adding support for other architectures, such as AMD and ARM, will require manual effort. Specifically, one would have to identify the (possibly undocumented) microarchitectural components whose state might affect the interaction with the cache, and manually disable or reset them between queries.

Likewise, CACHEQUERY currently runs on top of a fully-fledged Linux kernel. While facilitating development, this adds unnecessary complexity and non-determinism. Using a custom unikernel could provide a better suited environment for our experiments.

\subsection{Explaining policies}

In this section, we present our approach for synthesizing explanations of replacement policies in the form of high-level programs, starting from our automata models.

Policy explanations. We explain replacement policies in terms of four simpler rules: (a) a promotion rule describing how the control state is updated whenever there is a cache hit, (b) an eviction rule describing how to select the cache line to evict, (c) an insertion rule describing how the control state is updated whenever there is a cache miss, and (d) a normalization rule describing how to normalize the control state

\footnotetext{
${ }^{3}$ That is, movabs rax, qword [address] (or in binary 0x48 0xa1 <imm>).
} 


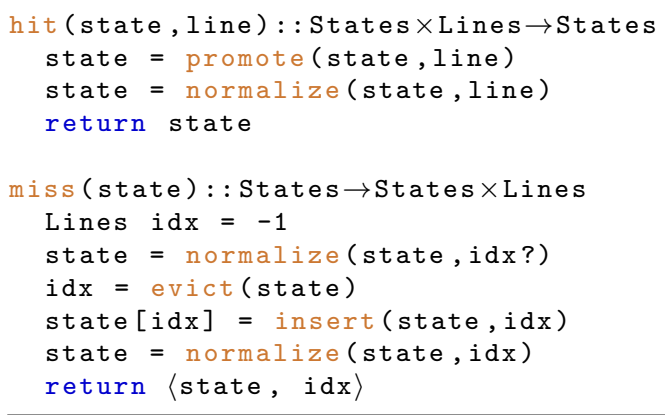

Figure 5.5: Hit and miss templates using several generators.

before or after a hit or a miss ${ }^{4}$. We borrow these terms from policy proposals from the hardware community [24].

Explanation template. The main component of our synthesis approach is a programlevel template for explanations, which is defined in terms of promotion, eviction, insertion, and normalization rules:

The template models control states as arrays mapping cache lines to their so-called ages. The concrete age values (of type Nat) are left as holes to be instantiated during the synthesis. Additionally, the template consists of two functions (see Figure 5.5):

The function hit describes how the control state is updated whenever there is a cache hit. The function takes as input a control state state and a cache line line, updates the control state using the promotion rule, normalizes it, and returns the new state.

In contrast, the function miss modifies the control state in case of a cache miss. The function takes as input a control state state, normalizes it, detects the cache line idx to evict using the eviction rule, updates the age of the evicted line using the insertion rule, and finally normalizes again the ages.

We remark that our templates - with promotion, eviction, insertion, and normalization rules - formalize well-known concepts and building blocks used by cache designers [24]

Generators. Our template specifies several generators for the rules. Generators are programs with holes that can be instantiated during synthesis. Each of the holes can be instantiated with expressions generated from specific grammars, which constraint the synthesis' search space. To illustrate, Figure 5.6 shows a generator for the promotion rule.

The generator takes as input a control state and a cache line and returns the updated control state. The updated state is derived by first conditionally modifying the age of the accessed line and later iterating over the remaining cache line and conditionally updating them.

All conditions and update expressions are encoded as holes that refer to template variables. For instance, the hole ??\{boolExpr(state[pos],state[i])\} can be instantiated

\footnotetext{
${ }^{4}$ Normalization is used in some policies to preserve control state invariants. For example, MRU updates the control state after a hit if all lines have age 0 .
} 


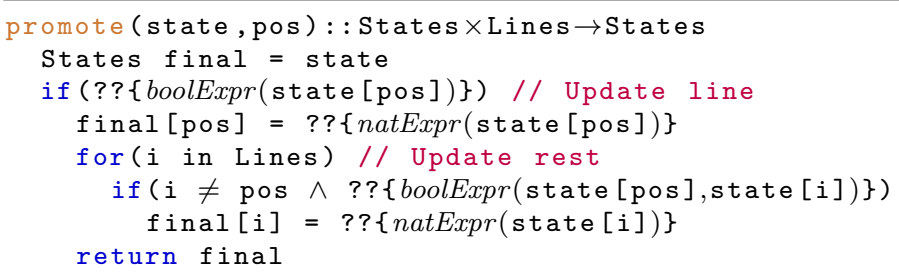

Figure 5.6: Generator example defining the grammar used for the promotion rule.

with a conjunction of equalities and inequalities that refer to natural numbers and to state[pos] and state[i], whereas ??\{natExpr(state[i])\} can be instantiated with an arbitrary sequence of additions and subtractions that refer to natural numbers and to state [i].

In general, our grammar generators can refer to constants, line indices (like pos and $i$ in the promote example), and ages (like state[pos] in the promote example). We also implement a simplified version of our generators that (1) fix the normalize rule to the identity function, and (2) restrict the grammar to only refer to constants and ages.

Constraints. Given a policy $\mathbf{P}$, we construct a formula $\varphi_{\mathbf{P}}$ encoding $\mathbf{P}$ 's transition relation $\rightarrow$ in terms of our template's hit and miss functions. In our encoding, we associate P's control states with logical variables. Concretely, we map each control state $\mathbf{c s}_{\mathbf{i}}$ in $\mathbf{P}$ to a corresponding variable $\mathrm{cs}_{i}$. The constraint $\varphi_{\mathbf{P}}$ is defined as follows, where $\mathbf{c s}_{\mathbf{1}}, \ldots, \mathbf{c s}_{\mathbf{m}}$ are all $P$ 's control states:

$$
\begin{aligned}
& \exists \operatorname{cs}_{1}, \ldots, \operatorname{cs}_{m} . \bigwedge_{1 \leq i, j \leq|P| \wedge i \neq j} \operatorname{cs}_{i} \neq \operatorname{cs}_{j} \wedge \\
& \bigwedge_{\delta\left(\mathbf{c s}_{\mathbf{k}}, \mathbf{L n}(\mathbf{i})\right)=\mathbf{c s}_{\mathbf{l}}} \operatorname{hit}\left(\operatorname{css}_{k}, i\right)=\operatorname{cs} l \wedge \\
& \bigwedge \quad \operatorname{miss}\left(\operatorname{cs}_{k}\right)=\left\langle\operatorname{cs}_{l}, i\right\rangle \\
& \delta\left(\mathbf{c s}_{\mathbf{k}}, \mathbf{E v c t}\right)=\mathbf{c s}_{\mathbf{l}} \wedge \\
& \lambda\left(\operatorname{cs}_{\mathbf{k}}, \mathbf{E v c t}\right)=\mathbf{i}
\end{aligned}
$$

The existential quantification and the first conjunct ensure that there are $m$ concrete control states (one per control state in $\mathbf{P}$ ). The second and third conjuncts ensure that the hit and miss functions behave as specified by $\mathbf{P}$.

Synthesis. To synthesize an explanation for a learned policy $\mathbf{P}$, we query a syntaxguided synthesis solver for an instance of our template that satisfies the constraint $\varphi_{\mathbf{P}}$. The solver, then, either returns a program $\operatorname{Prg}$ that instantiates the holes in the template in a way that satisfy $\varphi_{\mathbf{P}}$, or terminates without finding a model (in case our template cannot represent $\mathbf{P}$ ).

Example 5. Let $\mathbf{P}$ be the LRU policy with associativity 2 given in Example 2. The 
constraint $\varphi_{\mathbf{P}}$ is as follows:

$$
\begin{aligned}
& \exists \operatorname{cs}_{0}, \operatorname{cs}_{1} \cdot \operatorname{cs}_{0} \neq \operatorname{cs}_{1} \wedge \operatorname{hit}\left(\operatorname{cs}_{0}, 1\right)=\operatorname{cs}_{0} \wedge \\
& \quad h i t\left(\operatorname{cs}_{0}, 0\right)=\operatorname{cs}_{1} \wedge \text { hit }\left(\operatorname{cs}_{1}, 0\right)=\operatorname{cs}_{1} \wedge \text { hit }\left(\operatorname{cs}_{1}, 1\right)=\operatorname{cs}_{0} \wedge \\
& \operatorname{miss}\left(\operatorname{cs}_{0}\right)=\left\langle\operatorname{cs}_{1}, 0\right\rangle \wedge \operatorname{miss}\left(\operatorname{cs}_{1}\right)=\left\langle\operatorname{cs}_{0}, 1\right\rangle .
\end{aligned}
$$

Whenever the solver synthesizes a program that satisfy the given constraints, we can lift the correctness guarantee of our approach also to the synthesized program Prg. Indeed, the solver's soundness, the template's determinism, and the constraint $\varphi_{\mathbf{P}}$ ensure that $\operatorname{Prg}$ behaves exactly as the learned policy $\mathbf{P}$ on the concrete control states.

Limitations. Although our templates support a large class of policies (see Section 5.7), they cannot explain arbitrary policies. For instance, we model control states by associating an age to each cache line. Hence, policies with a global control state, such as PLRU, are not supported. Similarly, policies that do not follow the structure of the promotion, eviction, insertion, and normalization rules are not supported.

\subsection{Case Study: Learning from Software-Simulated Caches}

This section reports on a case study where we use POLCA to learn well-known replacement policies via software-simulated caches implementing such policies.

This case study's goals is to evaluate POLCA's efficiency and scalability across different classes of replacement policies, without the overhead introduced by interacting with real hardware. This case study also provides a basis for comparing PoLCA with prior approaches [96, 97].

Setup. We implemented software-simulated caches (parametric in the cache's associativity) for 7 commonly used replacement policies: First In First Out (FIFO), Least Recently Used (LRU), Pseudo-LRU (PLRU) [102], Most Recently Used (MRU) [103], LRU Insertion Policy (LIP) [106], and HP and FP variants of Static Re-reference Interval Prediction (SRRIP) [24] with 4 ages. We simulate the policies with associativity ranging from 2 to $16 .^{5}$ For each policy and associativity, we use PolCA to learn the policy with a timeout of 36 hours. We record the time needed to learn the automaton and the learned automaton's number of states. In our experiments, we set the test suite depth $k$ to 1 (Section 5.2.4), which proves sufficient for discovering counterexamples.

Results. Table 5.2 reports the time taken by PoLCA to learn the policies and the number of states of the resulting automata. We highlight the following:

\footnotetext{
${ }^{5}$ Some policies constraint the possible associativities. For instance, PLRU policies are welldefined only for associativities that are powers of 2 .
} 


\begin{tabular}{|c|c|c|c|}
\hline Policy & Assoc. & \# States & Time \\
\hline \multirow{8}{*}{ FIFO } & 2 & 2 & $0 \mathrm{~h} 0 \mathrm{~m} 0.14 \mathrm{~s}$ \\
\hline & 4 & 4 & $0.12 \mathrm{~s}$ \\
\hline & 6 & 6 & $0.14 \mathrm{~s}$ \\
\hline & 8 & 8 & $0.15 \mathrm{~s}$ \\
\hline & 10 & 10 & $0.14 \mathrm{~s}$ \\
\hline & 12 & 12 & $0.23 \mathrm{~s}$ \\
\hline & 14 & 14 & $0.31 \mathrm{~s}$ \\
\hline & 16 & 16 & $0 \mathrm{~h} 0 \mathrm{~m} 0.38 \mathrm{~s}$ \\
\hline \multirow{3}{*}{$L R U$} & 2 & 2 & $0 \mathrm{~h} 0 \mathrm{~m} 0.10 \mathrm{~s}$ \\
\hline & 4 & 24 & $0 \mathrm{~h} 0 \mathrm{~m} 0.22 \mathrm{~s}$ \\
\hline & 6 & 720 & $0 \mathrm{~h} 0 \mathrm{~m} 32.70 \mathrm{~s}$ \\
\hline \multirow{4}{*}{$P L R U$} & 2 & 2 & $0.10 \mathrm{~s}$ \\
\hline & 4 & 8 & $0.22 \mathrm{~s}$ \\
\hline & 8 & 128 & $1.46 \mathrm{~s}$ \\
\hline & 16 & 32768 & $34 \mathrm{~h} 18 \mathrm{~m} 25 \mathrm{~s}$ \\
\hline \multirow{6}{*}{$M R U$} & 2 & 2 & $0 \mathrm{~h} 0 \mathrm{~m} 0.10 \mathrm{~s}$ \\
\hline & 4 & 14 & $0 \mathrm{~h} 0 \mathrm{~m} 0.16 \mathrm{~s}$ \\
\hline & 6 & 62 & $0 \mathrm{~h} 0 \mathrm{~m} 0.61 \mathrm{~s}$ \\
\hline & 8 & 254 & $0 \mathrm{~h} 0 \mathrm{~m} 8.82 \mathrm{~s}$ \\
\hline & 10 & 1022 & $0 \mathrm{~h} 5 \mathrm{~m} 58 \mathrm{~s}$ \\
\hline & 12 & 4094 & $3 \mathrm{~h} 59 \mathrm{~m} 20 \mathrm{~s}$ \\
\hline \multirow{3}{*}{$L I P$} & 2 & 2 & $0 \mathrm{~h} 0 \mathrm{~m} 0.10 \mathrm{~s}$ \\
\hline & 4 & 24 & $0 \mathrm{~h} 0 \mathrm{~m} 0.26 \mathrm{~s}$ \\
\hline & 6 & 720 & $0 \mathrm{~h} 0 \mathrm{~m} 31.97 \mathrm{~s}$ \\
\hline \multirow{3}{*}{ SRRIP-HP } & 2 & 12 & $0 \mathrm{~h} 0 \mathrm{~m} 0.16 \mathrm{~s}$ \\
\hline & 4 & 178 & $0 \mathrm{~h} 0 \mathrm{~m} 1.46 \mathrm{~s}$ \\
\hline & 6 & 2762 & $0 \mathrm{~h} 9 \mathrm{~m} 38 \mathrm{~s}$ \\
\hline \multirow{3}{*}{$S R R I P-F P$} & 2 & 16 & $0 \mathrm{~h} 0 \mathrm{~m} 0.19 \mathrm{~s}$ \\
\hline & 4 & 256 & $0 \mathrm{~h} 0 \mathrm{~m} 7.27 \mathrm{~s}$ \\
\hline & 6 & 4096 & $2 \mathrm{~h} 30 \mathrm{~m} 51 \mathrm{~s}$ \\
\hline
\end{tabular}

Table 5.2: Learning policies from software-simulated caches (with 36 hours timeout). 
- Except for FIFO, the learning time grows roughly exponentially with associativity. POLCA learns FIFO and PLRU up to associativity 16.

- Prior approaches for permutation-based policies [97] can learn only FIFO, LRU, and PLRU, from our experimental setup. In contrast, PoLCA learns policies such like MRU, LIP, SRRIP-HP, and SRRIP-FP (up to associativities 12, 6, 6, and 6 respectively).

- Prior general purpose approaches [96] learn MRU only up to associativity 5 and timeout after 72 hours for larger associativities. In contrast, POLCA learns MRU up to associativity 12 and takes 600 milliseconds for associativity 6 .

Alternatives approach exists that leverage different heuristics, like random walks, for a deeper counterexample exploration. These approaches generally enable faster hypothesis refinement, and hence better performance. However, we opted for a default and deterministic setup, and leave a more thorough performance evaluation for future work.

Platform. We run all experiments on a Linux virtual machine (kernel 4.9.0-8-amd64) with Debian 9.0, Java OpenJDK v1.8.0_222, running on a Xeon Gold 6154 CPU (with 72 virtual cores), and 64 GB of RAM. We execute the experiments in parallel using a single virtual core for each policy.

\subsection{Case Study: Learning from Hardware}

This section reports on a case study where we use POLCA and CACHEQueRY to learn policies from real hardware. This case study's goals are (1) to determine whether POLCA can learn policies directly from hardware using CACHEQUERY as an interface, and (2) to understand the additional challenges involved with learning policies from hardware.

\subsubsection{Setup}

We analyze the L1, L2, and L3 caches of the Intel i7-4790 (Haswell), i5-6500 (Skylake), and i7-8550U (Kaby Lake) processors (see Table 5.3 for the specifications). For each processor and cache level, we use POLCA to learn the policy while using CACHEQUeRY as an interface to the hardware cache.

We encounter the following challenges:

- For some policies, PolCA does not scale to the large associativities used in L3 caches. To overcome this, we use Intel's CAT technology [114] to virtually reduce L3 associativity to 4 for the i5-6500 (Skylake) and i7-8550U (Kaby Lake) processors. CAT is not supported by i7-4790 (Haswell).

- Modern L3 caches often implement adaptive replacement policies [79, 106, 24], where separate groups of leader cache sets implement distinct replacement policies and the remaining follower sets switch between these policies dynamically. We 
Chapter 5. Learning Replacement Policies from Hardware Caches

\begin{tabular}{crrrr}
\hline CPU & Cache level & Assoc. & Slices & Sets per slice \\
\hline \multirow{2}{*}{ 7-4790 } & L1 & 8 & 1 & 64 \\
(Haswell) & L2 & 8 & 1 & 512 \\
& L3 & 16 & 4 & 2048 \\
\hline \multirow{2}{*}{ (S-6500 } & L1 & 8 & 1 & 64 \\
& L2 & 4 & 1 & 1024 \\
& L3 & 12 & 8 & 1024 \\
i7-8550U & L1 & 8 & 1 & 64 \\
Kaby Lake) & L2 & 4 & 1 & 1024 \\
& L3 & 16 & 8 & 1024 \\
\hline
\end{tabular}

Table 5.3: Processors' specifications [2, 3, 4].

\begin{tabular}{|c|c|c|c|c|c|c|}
\hline CPU & Level & Assoc. & Sets & States & Policy & Reset Seq. \\
\hline \multirow{4}{*}{$\begin{array}{l}i 7-4790 \\
\text { (Haswell) }\end{array}$} & L1 & 8 & $0-63$ & 128 & PLRU & @ @ \\
\hline & L2 & 8 & $0-511$ & 128 & PLRU & $\mathrm{F}+\mathrm{R}$ \\
\hline & L3 & 16 & $\begin{array}{l}512-575 \text { (only slice } \\
0 \text { ) }\end{array}$ & - & - & - \\
\hline & & & $\begin{array}{l}768-831 \text { (only slice } \\
0 \text { ) }\end{array}$ & - & - & - \\
\hline \multirow{4}{*}{$\begin{array}{l}i 5-6500 \\
(\text { Skylake) }\end{array}$} & L1 & 8 & $0-63$ & 128 & PLRU & $\mathrm{F}+\mathrm{R}$ \\
\hline & L2 & 4 & $0-1023$ & 160 & New1 & D C B A @ \\
\hline & L3 & $4^{\dagger}$ & 033132165264297 & 175 & New2 & $\mathrm{F}+\mathrm{R}$ \\
\hline & & & $\begin{array}{lllll}396 & 429 & 528 & 561 & 660 \\
693 & 792 & 825 & 924 & 957\end{array}$ & & & \\
\hline \multirow{5}{*}{$\begin{array}{c}i 7-8550 U \\
(\text { Kaby Lake) }\end{array}$} & L1 & 8 & $0-63$ & 128 & PLRU & $\mathrm{F}+\mathrm{R}$ \\
\hline & L2 & 4 & $0-1023$ & 160 & New1 & D C B A @ \\
\hline & L3 & $4^{\dagger}$ & $\begin{array}{llllll}0 & 33 & 132 & 165 & 264 & 297\end{array}$ & 175 & New2 & $\mathrm{F}+\mathrm{R}$ \\
\hline & & & $\begin{array}{lllll}396 & 429 & 528 & 561 & 660\end{array}$ & & & \\
\hline & & & 693792825924957 & & & \\
\hline
\end{tabular}

Table 5.4: Results of learning policies from hardware caches. $\dagger$ indicates that the associativity has been virtually reduced using CAT. The 'Sets' column specifies the analyzed cache sets (unless otherwise specified, the findings apply to all slices). $\mathrm{F}+\mathrm{R}$ denotes the use of Flush+Refill to reset the cache set state.

only learn leader sets' policies. We later detail how we identify leader sets with CacheQuery.

- Our membership oracle Polca (Algorithm 3) relies on the assumption that traces are executed from a fixed initial state. In practice, this leads to a bootstrapping problem: knowing the reset sequence (i.e., a sequence of memory accesses that brings the cache into a fixed initial state) is a prerequisite for learning the policy, but computing the reset sequence requires knowledge about the policy itself. On many CPUs, cache sets can be reset by Flush+Refill, i.e., by invalidating the entire content (with clflush or wbinvd instructions) and accessing associativitymany different blocks (with the ' $\mathrm{C}$ ' MBL macro). For CPUs where this is not the 
case, we manually identify reset sequences for each cache (see Table 5.4). This is enabled by the fact that incorrect reset sequences lead to nondeterministic behavior (equal prefixes produce different outputs), which triggers errors in the learning algorithm.

Platform. We run CACHEQUERY on three different machines equipped with the three processors. Additionally, we run POLCA on the same platform described in Section 5.5. The communication between PolcA and CACheQuery happens over SSH in a local network.

\subsubsection{Results}

Learned policies. Table 5.4 summarizes the learned policies. We highlight the following findings:

- For all processors' L1 caches and for Haswell's L2 cache, PolCA learns the same policy, that is, a tree-based PLRU policy. We identified this policy by checking its equivalence to a manually implemented PLRU automaton. This result confirms common folklore that Intel processors implement PLRU in their L1 policy.

- For Skylake's and Kaby Lake's L2 caches, Polca learns a previously undocumented policy. This policy is indicated as New1 in Table 5.4, and we further discuss it in Section 5.7.

- For Skylake's and Kaby Lake's L3 caches, Polca learns a previously undocumented policy for the leader sets. This policy is indicated as New2 in Table 5.4, and we further discuss it in Section 5.7. Additionally, we confirm the mapping of Skylake's leader sets described in Chapter 4, and discover that Kaby Lake follows the same mapping.

- For Haswell's L3 cache, Polca cannot learn the replacement policy. This is due to (1) i7-4790 not supporting CAT, and (2) one of the leader sets showing a non-deterministic behavior.

Cost of learning from hardware. Learning policies from hardware caches comes with a significant overhead when compared with learning from software-simulated caches. This is due to (1) communication overhead between PolCA and CACHEQUERY, and (2) CACHEQUERY overhead for code generation and profiling. We separately analyze the impact of (1) and (2).

For (1), we compare the time needed to learn a PLRU policy with associativity 8 from a software-simulated cache and from CACHEQUERY where every MBL query hits the LevelDB cache (i.e., the results of the MBL queries on the real hardware have been precomputed). Learning from the software-simulated cache takes $1.46 \mathrm{~s}$ (see Table 5.2), while learning from CACHEQUERY takes $2247 \mathrm{~s}$, resulting in a 1500x overhead.

For (2), we measure the time taken to execute a single MBL query ' $\mathrm{C}$ M _ ?' across cache levels. The averaged query execution time (across 100 executions on the i5-6500 (Skylake) processor is $16 \mathrm{~ms}$ on L1, $11 \mathrm{~ms}$ on L2, and $20 \mathrm{~ms}$ on L3. We remark that learning the PLRU policy with associativity 8 requires more than 50'000 MBL queries. 


\subsubsection{Identifying Leader Sets}

Henry Wong[25] identifies an adaptive L3 cache on Intel's Ivy Bridge processors, and describes possible heuristics to detect the existence of leader sets.

In a similar way, we use CACHEQUERY to run several thrashing queries on a per set basis, obtaining the following results:

- Haswell i7-4790: sets $512-575$ in slice 0 implement a fixed policy susceptible to thrashing. While sets $768-831$ in slice 0 implement a fixed thrash resistant policy (that also seems to be not deterministic). The rest of cache sets follow the policy producing less misses.

- Skylake i5-6500: index bits satisfying $b_{9} \oplus b_{4}=b_{8} \oplus b_{3}=b_{7} \oplus b_{2}=b_{6} \oplus b_{1}=$ $b_{5} \oplus b_{0}=b_{1}=0$ implement a fixed policy susceptible to thrashing (New2). The rest seem to use an adaptive policy that behaves in a non-deterministic way.

- Kaby Lake i7-8550U: we observe the same behavior and set selection than Skylake i5-6500.

On Haswell, we confirm previous results reported by Reload+Refresh [98]. However, we remark that leader sets are only present in slice 0 , which is also clarified in nanoBench [115]. It is also worth mentioning that the ranges seem to be selected by comparing the index bits with some fixed bit masks: $\left(b_{10}, \ldots, b_{6}\right)=8$ and $\left(b_{10}, \ldots, b_{6}\right)=12$, respectively.

In Chapter 4, we identified sets with a fixed policy on Skylake, and argued that leader set influence did not cross slices. We extend this knowledge, including a description for Kaby Lake, and show that adaptivity in fact has effects across different slices, i.e., a single cache set leader producing lots of hits can affect all the follower sets in the cache.

Furthermore, we provide some observations regarding the adaptive policy implemented in the rest of sets from the L3 cache in Skylake and Kaby Lake. First, we observe another group of sets (those with index bits satisfying $b_{9} \oplus b_{4}=b_{8} \oplus b_{3}=$ $\left.b_{7} \oplus b_{2}=b_{6} \oplus b_{1}=b_{5} \oplus b_{0}=b_{1}=1\right)$ that change at a different rate than the majority. Second, it is possible to control the adaptive policies in 2 ways, by only interacting with the thrash-vulnerable fixed sets: (1) causing thrashing on them makes the adaptive policies to be more thrash resistant, i.e., @ M a M? produces a miss; (2) causing a lot of hits makes them tend towards the New2 policy. We remark that this interaction needs to happen concurrently, which might indicate some complex dueling mechanism that takes clock cycles into account. If the unknown adaptive policy is inspired by DRRIP [24], it could behave deterministically when completely saturated. However, we have not yet been able to learn it. Interestingly, the set selection we describe is very similar to that in [106], what increases our confidence in the above explanation.

\subsection{Case Study: Synthesizing Explanations}

This section reports on a case study where we use the synthesis approach from Section 5.4 to derive policy explanations for automata learned in Sections 5.5-5.6. This case 
study's goals are (1) to evaluate if our approach can explain the replacement policies learned in Sections 5.5-5.6, and (2) to determine whether the synthesized explanations can help in understanding previously undocumented policies.

\subsubsection{Setup}

We encoded our template (and all rules generators) from Section 5.4 in Sketch [105]. We use Sketch to synthesize explanations for all the policies from Section 5.5 (i.e., FIFO, LRU, PLRU, MRU, LIP, SRRIP-HP, and SRRIP-IP) and for the undocumented policies New1 and New2 from Section 5.6. In our experiments, we fix the associativity to 4 .

For all policies, we synthesize explanations using Sketch $^{6}$, and record the time needed to synthesize an explanation that satisfies our constraints. During synthesis, we bound both the size of natural numbers and the recursion depth of our grammar generators, for associativity 4 , we choose a size bound of 4 and recursion depth bound of 2. We explore the synthesis space incrementally until we find a solution or the space is exhausted. For each policy, we first try synthesizing an explanation using the simplified template from Section 5.5 (which we refer to as Simple template), and if we cannot synthesize a solution, then we try using the more general template from Section 5.5 (which we refer to as Extended template).

Platform. We run the experiments on the same platform as in Section 5.5 and use Sketch v1.7.5, with a single thread.

\subsubsection{Results}

Table 5.5 summarizes the results of our synthesis approach. We highlight the following findings:

1. Our approach successfully explains the FIFO, LRU, and LIP policies using the Simple template in less than $5 \mathrm{~s}$.

2. Our approach synthesize explanations for the MRU, SRRIP-HP, SRRIP-FP, New1, and New2 policies using the Extended template. The synthesis time varies (from $\sim 40 \mathrm{~s}$ for MRU to $\sim 4.5$ days for SRRIP-HP), but it is roughly correlated with the number of states.

3. Our current templates do not encompass PLRU. The main reason is that PLRU uses a tree-based data structure as global control state, rather than a local, per-line control state as in our templates. Supporting tree-based policies would require modifying our templates to handle a global state and extending our grammars with operators for the traversal of tree-based structures. Synthesis, however, did not successfully terminate in our initial experiments for this enhanced template. Thus, we opted in favor of simpler and more general templates that allow

\footnotetext{
${ }^{6}$ Sketch uses a random seed to explore the search space. Hence, Sketch might synthesize different explanations that satisfy the constraints. In our experiments, we fix the seed to --slv-seed 1337.
} 
us to explain a broader set of policies in reasonable time, even at the cost of not supporting special tree-based global policies like PLRU.

\begin{tabular}{ccrc}
\hline Policy & States & Template & Execution Time \\
\hline FIFO & 4 & Simple & $0 \mathrm{~h} 0 \mathrm{~m} 0.18 \mathrm{~s}$ \\
LRU & 24 & Simple & $0 \mathrm{~h} 0 \mathrm{~m} 0.81 \mathrm{~s}$ \\
PLRU & 8 & - & - \\
LIP & 24 & Simple & $0 \mathrm{~h} 0 \mathrm{~m} 4.36 \mathrm{~s}$ \\
MRU & 14 & Extended & $0 \mathrm{~h} 0 \mathrm{~m} 39.80 \mathrm{~s}$ \\
SRRIP-HP & 178 & Extended & $105 \mathrm{~h} 28 \mathrm{~m} 30 \mathrm{~s}$ \\
SRRIP-FP & 256 & Extended & $48 \mathrm{~h} 30 \mathrm{~m} 25 \mathrm{~s}$ \\
New1 & 160 & Extended & $9 \mathrm{~h} 36 \mathrm{~m} 9 \mathrm{~s}$ \\
New2 & 175 & Extended & $26 \mathrm{~h} 4 \mathrm{~m} 22 \mathrm{~s}$ \\
\hline
\end{tabular}

Table 5.5: Synthesizing explanations for policies (of associativity 4). In the Simple template, normalize is fixed to the identity function and the grammar for expressions is simpler. In contrast, the Extended template supports the normalize rule and has a more expressive expression grammar.

Explaining New1 and New2. Sketch successfully synthesize explanations for the previously undocumented policies New1 and New2 from Section 5.6. Below we provide a high-level description of the policies.

New1 policy is defined by:

- The initial control state is $\{3,3,3,0\}$.

- Promote: Set the accessed line's age to 0.

- Evict: Select the first line, starting from left, whose age is 3.

- Insert: Set the evicted line's age to 1.

- Normalize: After a hit or a miss, while there is no line with age 3, increase the age of all lines by 1 except for the just accessed/evicted line.

New2 policy is defined by:

- The initial control state is $\{3,3,3,3\}$.

- Promote: If the accessed line has age 1 set it to 0 , otherwise set it to 1.

- Evict: Select the first line, starting from left, whose age is 3.

- Insert: Set the evicted line's age to 1.

- Normalize: After a hit or miss, while there is no line with age 3, increase all lines by 1 . 


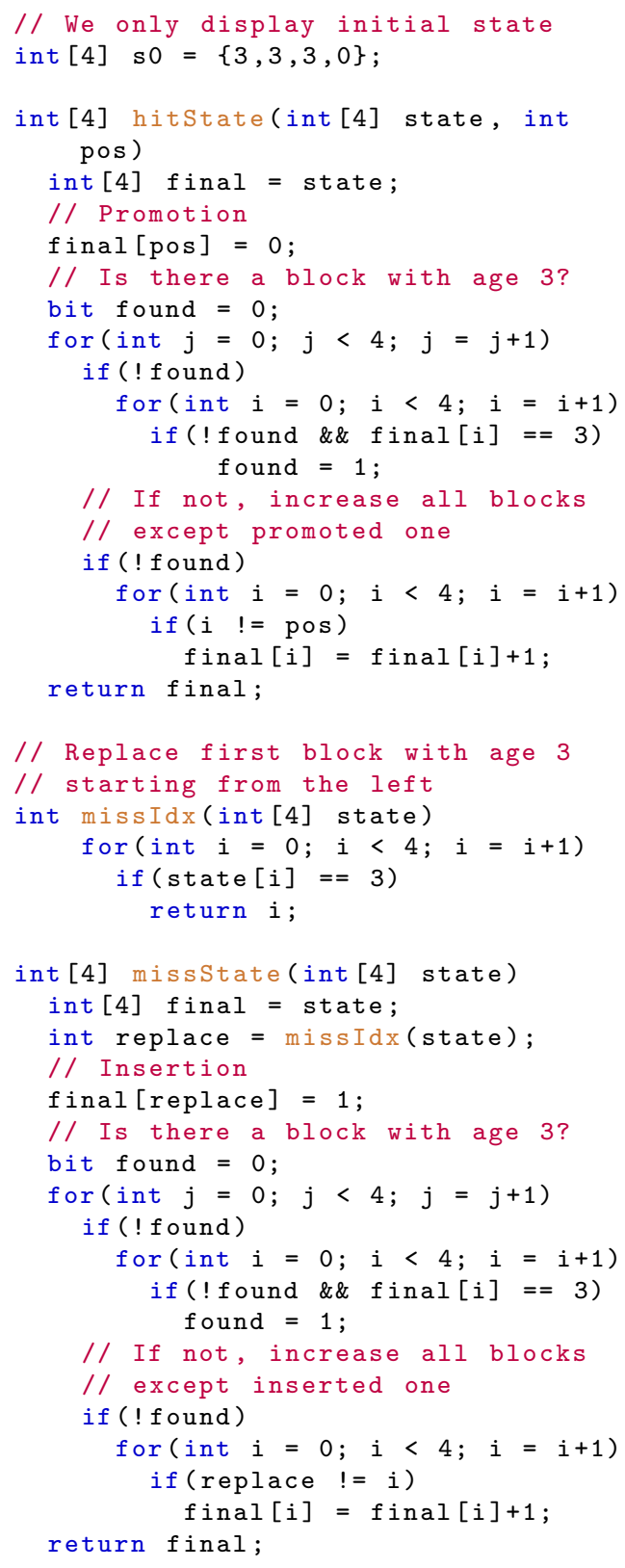

(a) Sketch solution for New1 undocumented policy.

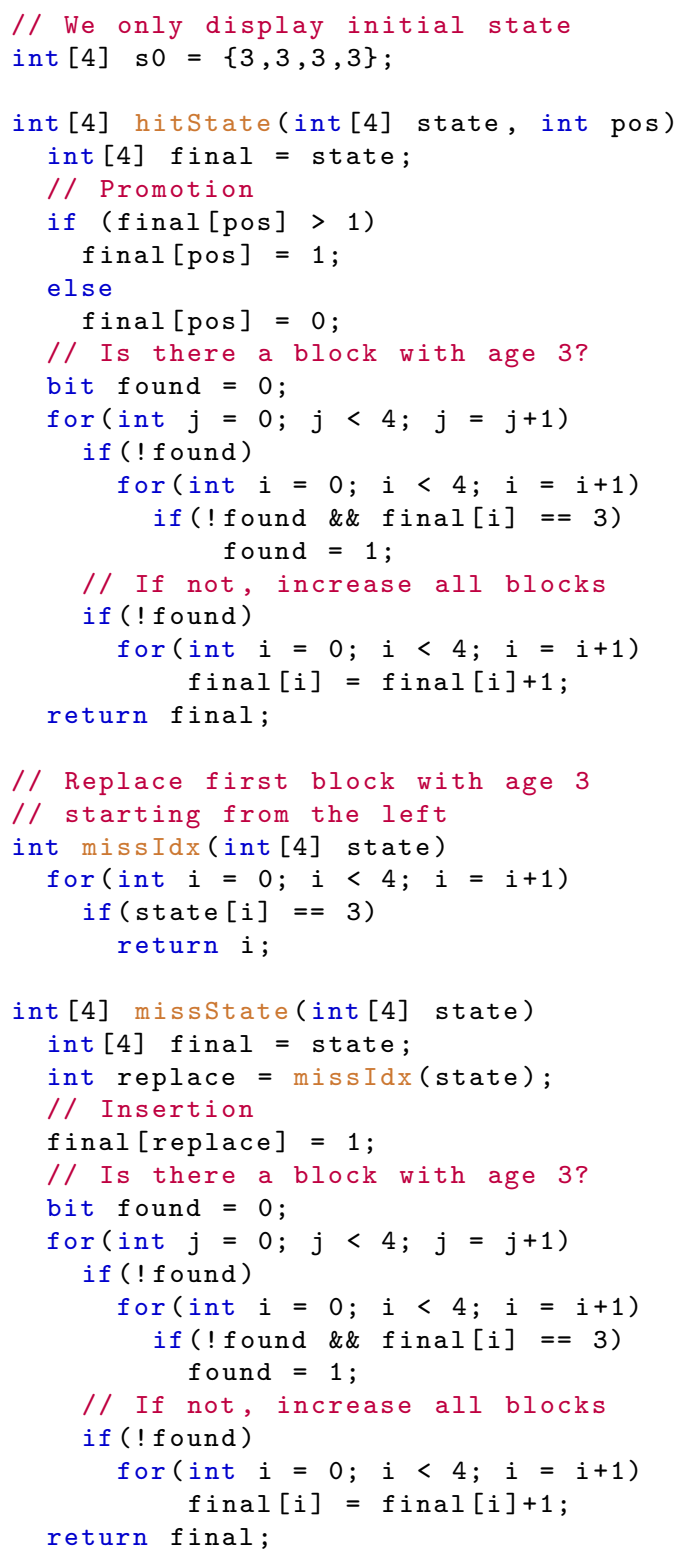

(b) Sketch solution for New2 undocumented policy.

Figure 5.7: Synthesized high-level programs for previously undocumented replacement policies using the Extended template.

In contrast to the automata models, our high-level representation allow us to compare the previously undocumented policies with known ones. Concretely, both New1 and New2 are variants of the SRRIP-HP policy, defined in [24]. The main difference appears in the normalization rule, where SRRIP-HP normalizes the ages (by increasing 
all ages by 1 while there is no line with age 3 ) only before a miss.

Figure 5.7 shows a complete cleaned up version, with minor variable renaming and layout adjustments, for the synthesized readable solutions describing the 2 previously undocumented cache replacement policies.

In a concurrent work, Andreas and Reineke [115] obtain the same results using a different approach, which again increases our confidence in the explanations.

\subsection{Discussion}

\section{Threats to validity}

- Hardware interface: CACHEQueRY employs several mechanisms (see Section 5.3.3) to eliminate noise and to provide an interface to a cache whose state is determined by explicit memory loads. However, a replacement policy could take into account hints from hardware prefetchers, different cache levels, or other partitions created by CAT [116]. We suppress these effects by design and thus potentially learn a restricted state machine.

- Automata learning: Our automata learning approach provides precise correctness guarantees (Corollary 4). These guarantees rely on two assumptions: (1) the policy under learning is a deterministic finite state machine, and (2) the test suite of depth $k$ is large enough to find counterexamples during the learning. Violating any of these assumptions may result in an incorrect policy.

Scalability Our approach can successfully learn policies up to associativity 16 (for software simulators, see Section 5.5) and 8 (for hardware caches, see Section 5.6). PolCA is key to make automata learning scale to this extent since it exploits dataindependence symmetries to significantly reduce the learning state-space. For many policies, however, scalability is still limited by the state-space's exponential growth with respect to associativity. Potential paths forward include identifying and exploiting further symmetries to reduce the state-space, learning abstractions rather than full models, or giving up on the correctness guarantees. Due to its modularity, our approach is well-suited for integrating such variants (and future improvements) in automata learning and program synthesis.

\subsection{Related Work}

Model learning. For related work on automata learning techniques for black-box systems we refer the interested reader to Vaandrager's survey paper [117].

Reverse-engineering cache policies. Abel and Reineke [97] design an efficient algorithm for learning permutation base replacement policies, a class of policies that include LRU, PLRU, and FIFO. They use an adhoc approach to reverse engineer two variants of PLRU that employ randomization [99]. 
Guillem Rueda's master thesis [96] studies how register automata learning can serve to learn a broader class of replacement policies, in comparison to permutation based policies, including MRU. This is an interesting and novel approach, however, their method does not scale in practice (Section 5.5).

Wong [25] notices the use of adaptive policies in Intel's Ivy Bridge, and tries to identify the new implemented policies guided by recent papers [79, 24], without complete success.

In concurrent work, Abel and Reineke extend nanoBench [94, 115] to reverse engineer cache replacement policies. In contrast to our approach, they proceed by producing random sequences and comparing the results from hardware against a pool of more than 300 software-simulated caches. While this approach is less general and the results lack correctness guarantees, in practice, it proves highly efficient and accurate. They are able to target larger caches and to infer probabilistic replacement policies; and our results confirm several of their findings.

Security. Rowhammer.js [7] tests thousands of eviction strategies, memory access patterns with high eviction rate, in order to identify efficient strategies to mount a rowhammer attack from a web browser. Recent attacks $[92,116,118]$ show how detailed knowledge about the replacement policy state can leak information, in contrast to the common content based leak. Similarly, Briongos et al. [98] exploit a new cache sidechannel attack leveraging changes in the policy state to bypass mechanisms based on monitoring of cache misses. For this, they attempt to explain the behavior of the replacement policy on several modern Intel CPUs. While their description is not completely accurate, it is enough to prove their attack.

Detailed policy models, such as the ones we provide, enable one to systematically compute optimal eviction strategies, and to unveil new sophisticated cache attacks.

Custom kernels. Recent research projects have developed custom kernels and hypervisors for specialized tasks that require extremely high performance, precise measurements, or access to privileged modes. These environments provide complete control over the hardware improving testing and reproducibility. Some examples include angryOS [119], which has been used for reverse engineering microcode, or Sushi Roll [120], a highly deterministic kernel, initially designed for fuzzing, converted into a cycle-bycycle CPU introspection tool.

Implementing interfaces as CACHEQUERY on a custom kernel can provide a better environment for high performance and predictability, ultimately enabling the use of learning methods for other undocumented microarchitectural components, like prefetchers, branch predictors, or data buffers. 


\section{Conclusions \& Future Work}

\subsection{Conclusions}

This thesis provides novel results in the different topics presented in Chapter 1, and furthermore, poses new questions and research lines for future work.

On exploitation, we demonstrate that shared event loops in Chrome are vulnerable to side-channel attacks, and how a spy renderer can break confidentiality by monitoring the loop usage pattern of other processes with unprivileged JavaScript snippets. This work renovates its relevance in the context of multi-process browsers with Site Isolation [121] and SameSite cookies [122], where many common classes of attacks are being effectively mitigated. Under these settings, cross-origin-and-process leaks promise to become the next line of attack in the web. However, our approach is not only relevant to browsers, and the question of which other event-driven systems are susceptible to these kind of attacks is still open.

On quantification, we provide the first systematic study of the problem of finding minimal cache eviction, and break several countermeasures based on wrong assumptions about the attacker's capabilities. By connecting the problem with threshold-group testing and providing a novel algorithm, we not only improve the asymptotic cost from quadratic to linear, but also provide a practical speed up. Our results demonstrate the benefits of carefully studying well known building blocks, and provide the basis for research on principled countermeasures against microarchitectural attacks.

On models, we present a practical end-to-end solution for learning hardware cache replacement policies directly from timing measurements. In our experiments we successfully infer human-readable descriptions of cache replacement policies used in recent Intel processors, including 2 previously undocumented policies. Our contributions are two independent tools, ready to use in alternative workflows, such as advanced learning approaches [123, 124], or manual analysis.

\subsection{Future Work}

A more detailed analysis of the algorithmic implications of adaptive cache replacement policies for finding minimal eviction sets is out of the scope of this work. However, we 
briefly describe some ideas for future work:

- Controlling the policy. A better understanding of adaptivity mechanisms could be exploited to augment the number of followers that behave deterministically, and hence facilitate the reduction. For instance, once we find eviction sets for a leader set on Skylake, a parallel thread could produce hits on that set (or misses on another leader), ensuring that it keeps the lead.

- Group testing. Work on noisy group testing [125], or threshold group testing with gap [68], can provide useful tools for dealing with the uncertainty about the exact behavior of modern microarchitectures.

Another line of work we are currently developing is to quantify cache leakage using the automata models extracted in Chapter 5. For this, we leverage useful notions from automata theory, like synchronizing and distinguishing sequences, to compute the maximum capacity of cache channels. With this approach, we plan to provide useful metric for evaluating the security of different cache implementations.

Likewise, CACHEQUery, as a prototype, shows that it is possible to establish a civilized interaction with a complex microarchitectural component, thus enabling the use of off-the-shelf automatic learning techniques. We plan to extend this approach to cover other undocumented components, like hardware prefetchers or branch predictors. 


\section{Funding Acknowledgments}

This research was supported by a grant from Intel Corporation, Atracción de Talento Investigador grant 2018-T2/TIC-11732A, Ramón y Cajal fewlloship grants RYC-201416766 and RYC-2016-20281, Spanish projects TIN2015-70713-R DEDETIS, TIN201239391-C04-01 StrongSoft, and TIN2015-67522-C3-1-R TRACES, RTI2018-102043-BI00 SCUM and PGC2018-102210-B-I00 BOSCO, and Madrid regional projects S2013/ICE2731 N-GREENS and S2018/TCS-4339 BLOQUES. 


\section{Bibliography}

[1] B. Steffen, F. Howar, and M. Merten, Introduction to Active Automata Learning from a Practical Perspective, pp. 256-296. Springer Berlin Heidelberg, 2011.

[2] Intel, Intel 64 and IA-32 Architectures Optimization Reference Manual. Intel, 2018 .

[3] G. Irazoqui, T. Eisenbarth, and B. Sunar, "Systematic reverse engineering of cache slice selection in intel processors," in In Proceedings of the 18th EUROMICRO Conference on Digital System Design, 2015.

[4] C. Maurice, N. L. Scouarnec, C. Neumann, O. Heen, and A. Francillon, "Reverse engineering intel last-level cache complex addressing using performance counters," in Research in Attacks, Intrusions, and Defenses - 18th International Symposium, RAID 2015, Kyoto, Japan, November 2-4, 2015, Proceedings, pp. 48-65, 2015.

[5] J. Spolsky, "The law of leaky abstractions," November, 11 2002. https://www. joelonsoftware.com/2002/11/11/the-law-of-leaky-abstractions/.

[6] M. Seaborn and T. Dullien, "Exploiting the DRAM rowhammer bug to gain kernel privileges," in Black Hat, 2015.

[7] Daniel Gruss and Clémentine Maurice and Stefan Mangard, "Rowhammer.js: A Remote Software-Induced Fault Attack in JavaScript," in DIMVA, Springer, 2016.

[8] P. C. Kocher, "Timing attacks on implementations of diffie-hellman, rsa, dss, and other systems," in Advances in Cryptology - CRYPTO '96, (Berlin, Heidelberg), pp. 104-113, Springer Berlin Heidelberg, 1996.

[9] D. J. Bernstein, "Cache-timing attacks on AES," 2005. https://cr.yp.to/ antiforgery/cachetiming-20050414.pdf. 
[10] N. S. ecurity Agency, "TEMPEST: A Signal Problem," 1972. Mirror to declassified document: http://cryptome.org/nsa-tempest.pdf.

[11] B. Carrara, Air-gap covert channels. PhD thesis, Université d'Ottawa/University of Ottawa, 2016.

[12] P. C. Kocher, J. Jaffe, and B. Jun, "Differential power analysis," in Proceedings of the 19th Annual International Cryptology Conference on Advances in Cryptology, CRYPTO '99, (Berlin, Heidelberg), p. 388-397, Springer-Verlag, 1999.

[13] P. Kocher, J. Horn, A. Fogh, D. Genkin, D. Gruss, W. Haas, M. Hamburg, M. Lipp, S. Mangard, T. Prescher, M. Schwarz, and Y. Yarom, "Spectre attacks: Exploiting speculative execution," in IEEE Symposium on Security and Privacy (SP), 2019.

[14] M. Lipp, M. Schwarz, D. Gruss, T. Prescher, W. Haas, A. Fogh, J. Horn, S. Mangard, P. Kocher, D. Genkin, Y. Yarom, and M. Hamburg, "Meltdown: Reading kernel memory from user space," in 27th USENIX Security Symposium (USENIX Security 18), (Baltimore, MD), pp. 973-990, USENIX Association, Aug. 2018.

[15] D. Kohlbrenner and H. Shacham, "Trusted browsers for uncertain times," in 25th USENIX Security Symposium (USENIX Security 16), (Austin, TX), pp. 463-480, USENIX Association, Aug. 2016.

[16] M. Schwarz, C. Maurice, D. Gruss, and S. Mangard, "Fantastic timers and where to find them: High-resolution microarchitectural attacks in javascript," in $F i$ nancial Cryptography and Data Security (A. Kiayias, ed.), (Cham), pp. 247-267, Springer International Publishing, 2017.

[17] R. McIlroy, J. Sevcík, T. Tebbi, B. L. Titzer, and T. Verwaest, "Spectre is here to stay: An analysis of side-channels and speculative execution," CoRR, vol. abs/1902.05178, 2019.

[18] D. Brumley and D. Boneh, "Remote timing attacks are practical," in Proceedings of the 12th Conference on USENIX Security Symposium - Volume 12, SSYM'03, (USA), p. 1, USENIX Association, 2003.

[19] S. A. Crosby, D. S. Wallach, and R. H. Riedi, "Opportunities and limits of remote timing attacks," ACM Trans. Inf. Syst. Secur., vol. 12, pp. 17:1-17:29, Jan. 2009.

[20] A. Abel, F. Benz, J. Doerfert, B. Dörr, S. Hahn, F. Haupenthal, M. Jacobs, A. H. Moin, J. Reineke, B. Schommer, and R. Wilhelm, "Impact of resource sharing on performance and performance prediction: A survey," in CONCUR 2013 - Concurrency Theory, (Berlin, Heidelberg), pp. 25-43, Springer Berlin Heidelberg, 2013.

[21] T. Dullien, "MitiGator. The well-intentioned, but short-sighted and not terribly effective alligator, always working to make exploitation harder." https: //twitter.com/halvarflake/status/836492121339211776. 
[22] A. Barth, C. Jackson, C. Reis, T. Team, et al., "The security architecture of the chromium browser." http://www.adambarth.com/papers/2008/ barthjackson-reis.pdf, 2008.

[23] C. Reis and S. D. Gribble, "Isolating web programs in modern browser architectures," in EuroSys, ACM, 2009.

[24] A. Jaleel, K. B. Theobald, S. C. Steely, Jr., and J. Emer, "High Performance Cache Replacement Using Re-reference Interval Prediction (RRIP)," SIGARCH Comput. Archit. News, vol. 38, pp. 60-71, June 2010.

[25] H. Wong, "Intel Ivy Bridge Cache Replacement Policy," January 2013.

[26] "The linux kernel archives: Transparent huge pages." https: //www .kernel.org/ doc/Documentation/vm/transhuge.txt, 2017.

[27] "HTML Living Standard." https://html.spec.whatwg.org/. Accessed: 201705-24.

[28] B. W. Lampson, "A note on the confinement problem," Communications of the $A C M$, vol. 16, no. 10, pp. 613-615, 1973.

[29] D. Bernstein, "Cache-timing attacks on AES." https://cr.yp.to/ antiforgery/cachetiming-20050414.pdf, 2005.

[30] D. A. Osvik, A. Shamir, and E. Tromer, "Cache attacks and countermeasures: the case of AES," in CT-RSA, Springer, 2006.

[31] Y. Yarom and K. Falkner, "FLUSH+RELOAD: A high resolution, low noise, L3 cache side-channel attack," in USENIX Security Symposium, 2014.

[32] S. Kadloor, N. Kiyavash, and P. Venkitasubramaniam, "Mitigating timing side channel in shared schedulers," IEEE/ACM Trans. Netw., vol. 24, no. 3, pp. 15621573, 2016.

[33] S. Jana and V. Shmatikov, "Memento: Learning secrets from process footprints," in $S S P$, IEEE, 2012.

[34] M. A. Hogye, C. T. Hughes, J. M. Sarfaty, and J. D. Wolf, "Analysis of the feasibility of keystroke timing attacks over ssh connections." http://www.cs. virginia.edu/ evans/cs588-fall2001/projects/reports/team4.pdf, 2001.

[35] K. Zhang and X. Wang, "Peeping tom in the neighborhood: Keystroke eavesdropping on multi-user systems," in USENIX Security, USENIX Association, 2009 .

[36] D. Gruss, R. Spreitzer, and S. Mangard, "Cache template attacks: Automating attacks on inclusive last-level caches," in USENIX Security, USENIX Association, 2015 .

[37] D. X. Song, D. Wagner, and X. Tian, "Timing Analysis of Keystrokes and Timing Attacks on SSH," in USENIX Security, USENIX Association, 2001. 
[38] E. Bosman, K. Razavi, H. Bos, and C. Giuffrida, "Dedup Est Machina: Memory Deduplication as an Advanced Exploitation Vector," in SSP, IEEE, 2016.

[39] M. V. Pedersen and A. Askarov, "From Trash to Treasure: Timing-sensitive Garbage Collection," in SSP, IEEE, 2017.

[40] "Understanding about:tracing results." https://www.chromium.org/ developers/how-tos/trace-event-profiling-tool/trace-event-reading. Accessed: 2017-02-16.

[41] D. J. Berndt and J. Clifford, "Using dynamic time warping to find patterns in time series.," in KDD workshop, AAAI Press, 1994.

[42] T. Giorgino, "Computing and visualizing dynamic time warping alignments in $\mathrm{r}$ : The dtw package," JSS, vol. 31, no. 7, pp. 1-24, 2009.

[43] Y. Sakurai, C. Faloutsos, and M. Yamamuro, "Stream monitoring under the time warping distance," in ICDE, IEEE, 2007.

[44] "Covert channels in the sop." https://github.com/cgvwzq/ sop-covert-channels. Accessed: 2017-02-16.

[45] T. Zhang, Y. Zhang, and R. B. Lee, "CloudRadar: A Real-Time Side-Channel Attack Detection System in Clouds," in RAID, Springer, 2016.

[46] B. Gras, K. Razavi, E. Bosman, H. Bos, and C. Giuffrida, "ASLR on the Line: Practical Cache Attacks on the MMU," in NDSS, The Internet Society, 2017.

[47] "Isolation explainer." https://wicg.github.io/isolation/explainer.html, 2016. Accessed: 2017-05-24.

[48] E. W. Felten and M. A. Schneider, "Timing attacks on web privacy," in $C C S$, ACM, 2000.

[49] A. Bortz and D. Boneh, "Exposing private information by timing web applications," in $W W W, \mathrm{ACM}, 2007$.

[50] T. Van Goethem, W. Joosen, and N. Nikiforakis, "The Clock is Still Ticking: Timing Attacks in the Modern Web," in CCS, ACM, 2015.

[51] N. Gelernter and A. Herzberg, "Cross-Site Search Attacks," in CCS, ACM, 2015.

[52] R. Kotcher, Y. Pei, P. Jumde, and C. Jackson, "Cross-origin pixel stealing: timing attacks using CSS filters," in CCS, ACM, 2013.

[53] P. Stone, "Pixel perfect timing attacks with html5 (white paper)." https://www . contextis.com/documents/2/Browser_Timing_Attacks.pdf, 2013.

[54] M. Andrysco, D. Kohlbrenner, K. Mowery, R. Jhala, S. Lerner, and H. Shacham, "On subnormal floating point and abnormal timing.," in SSP, IEEE, 2015. 
[55] Y. Oren, V. P. Kemerlis, S. Sethumadhavan, and A. D. Keromytis, "The Spy in the Sandbox: Practical Cache Attacks in JavaScript and Their Implications," in $C C S$, ACM, 2015.

[56] D. Gruss, C. Maurice, and S. Mangard, "Rowhammer.js: A remote softwareinduced fault attack in javascript," in DIMVA, Springer, 2016.

[57] J. M. Booth, "Not so incognito: Exploiting resource-based side channels in javascript engines." http://nrs.harvard.edu/urn-3:HUL. InstRepos : $17417578,2015$.

[58] E. Vela, "Matryoshka: Timing attacks against javascript applications in browsers." http://sirdarckcat.blogspot.com.es/2014/05/ matryoshka-web-application-timing.html, 2013.

[59] A. Panchenko, F. Lanze, J. Pennekamp, T. Engel, A. Zinnen, M. Henze, and K. Wehrle, "Website fingerprinting at internet scale," in NDSS, The Internet Society, 2016.

[60] J. Hayes and G. Danezis, "k-fingerprinting: A Robust Scalable Website Fingerprinting Technique," in USENIX Security, USENIX Association, 2016.

[61] Q. Sun, D. R. Simon, Y.-M. Wang, W. Russell, V. N. Padmanabhan, and L. Qiu, "Statistical identification of encrypted web browsing traffic," in SSP, IEEE, 2002.

[62] K. P. Dyer, S. E. Coull, T. Ristenpart, and T. Shrimpton, "Peek-a-Boo, I Still See You: Why Efficient Traffic Analysis Countermeasures Fail," in SSP, IEEE, 2012.

[63] Y. Yarom and K. Falkner, "FLUSH+RELOAD: A High Resolution, Low Noise, L3 Cache Side-Channel Attack," in 23rd USENIX Security Symposium (USENIX Security 14), (San Diego, CA), pp. 719-732, USENIX Association, 2014.

[64] F. Liu, Y. Yarom, Q. Ge, G. Heiser, and R. B. Lee, "Last-Level Cache SideChannel Attacks Are Practical," in Proceedings of the 2015 IEEE Symposium on Security and Privacy, SP '15, (Washington, DC, USA), pp. 605-622, IEEE Computer Society, 2015.

[65] G. Irazoqui, T. Eisenbarth, and B. Sunar, "S\$A: A Shared Cache Attack That Works Across Cores and Defies VM Sandboxing - and Its Application to AES," in Proceedings of the 2015 IEEE Symposium on Security and Privacy, SP '15, (Washington, DC, USA), pp. 591-604, IEEE Computer Society, 2015.

[66] Daniel Gruss and Clémentine Maurice and Stefan Mangard, "Rowhammer.js: A Remote Software-Induced Fault Attack in JavaScript," in DIMVA, Springer, 2016.

[67] D. G. Michael Schwarz, Moritz Lipp, "JavaScript Zero: Real JavaScript and Zero Side-Channel Attacks," in Network and Distributed System Security Symposium 2018 (NDSS'18), 2018. 
[68] P. Damaschke, Threshold Group Testing, pp. 707-718. Springer Berlin Heidelberg, 2006.

[69] Y. Oren, V. P. Kemerlis, S. Sethumadhavan, and A. D. Keromytis, "The Spy in the Sandbox: Practical Cache Attacks in JavaScript and Their Implications," in CCS, ACM, 2015.

[70] A. Abel and J. Reineke, "Reverse engineering of cache replacement policies in intel microprocessors and their evaluation," in IEEE International Symposium on Performance Analysis of Systems, 2014.

[71] J. Horn, "Reading privileged memory with a side-channel." https://googleprojectzero.blogspot.com.es/2018/01/ reading-privileged-memory-with-side.html, 2018.

[72] E. Bosman, K. Razavi, H. Bos, and C. Giuffrida, "Dedup Est Machina: Memory Deduplication as an Advanced Exploitation Vector," in IEEE Symposium on Security and Privacy, SP 2016, San Jose, CA, USA, May 22-26, 2016, pp. 9871004, 2016.

[73] B. Levin, "A representation for multinomial cumulative distribution functions," The Annals of Statistics, pp. 1123-1126, 1981.

[74] R. Dorfman, "The detection of defective members of large populations," The Annals of Mathematical Statistics, vol. 14, no. 4, pp. 436-440, 1943.

[75] T. H. Cormen, C. E. Leiserson, R. L. Rivest, and C. Stein, "Introduction to algorithms," Growth, vol. 5, no. A8, p. B1, 1989.

[76] D. Genkin, L. Pachmanov, E. Tromer, and Y. Yarom, "Drive-by key-extraction cache attacks from portable code." Cryptology ePrint Archive, Report 2018/119, 2018 .

[77] C. Maurice, N. Le Scouarnec, C. Neumann, O. Heen, and A. Francillon, "Reverse Engineering Intel Last-Level Cache Complex Addressing Using Performance Counters," in Proceedings of the 18th International Symposium on Research in Attacks, Intrusions and Defenses (RAID'15), November 2015.

[78] C. Maurice, M. Weber, M. Schwarz, L. Giner, D. Gruss, C. Alberto Boano, S. Mangard, and K. Römer, "Hello from the Other Side: SSH over Robust Cache Covert Channels in the Cloud," in Proceedings of the 24th Annual Network and Distributed System Security Symposium, NDSS, The Internet Society, February 2017.

[79] M. K. Qureshi, D. N. Lynch, O. Mutlu, and Y. N. Patt, "A Case for MLPAware Cache Replacement," SIGARCH Comput. Archit. News, vol. 34, no. 2, pp. 167-178, 2006.

[80] D. Gruss, R. Spreitzer, and S. Mangard, "Cache template attacks: Automating attacks on inclusive last-level caches," in 24th USENIX Security Symposium 
(USENIX Security 15), (Washington, D.C.), pp. 897-912, USENIX Association, 2015.

[81] Y. Kim, R. Daly, J. Kim, C. Fallin, J. H. Lee, D. Lee, C. Wilkerson, K. Lai, and O. Mutlu, "Flipping Bits in Memory Without Accessing Them: An Experimental Study of DRAM Disturbance Errors," in Proceeding of the 41st Annual International Symposium on Computer Architecuture, ISCA '14, pp. 361-372, IEEE Press, 2014.

[82] M. Oliverio, K. Razavi, H. Bos, and C. Giuffrida, "Secure Page Fusion with VUsion," in Proceedings of the 26th Symposium on Operating Systems Principles, Shanghai, China, October 28-31, 2017, pp. 531-545, 2017.

[83] M. Lipp, D. Gruss, R. Spreitzer, C. Maurice, and S. Mangard, "ARMageddon: Cache Attacks on Mobile Devices," in 25th USENIX Security Symposium (USENIX Security 16), (Austin, TX), pp. 549-564, USENIX Association, 2016.

[84] M. Schwarz, S. Weiser, D. Gruss, C. Maurice, and S. Mangard, Malware Guard Extension: Using SGX to Conceal Cache Attacks, pp. 3-24. Springer International Publishing, 2017.

[85] "L3 cache mapping on Sandy Bridge CPUs." http://lackingrhoticity. blogspot.com.es/2015/04/13-cache-mapping-on-sandy-bridge-cpus . html, 2015.

[86] R. Hund, C. Willems, and T. Holz, "Practical Timing Side Channel Attacks Against Kernel Space ASLR," in Proceedings of the 2013 IEEE Symposium on Security and Privacy, SP '13, (Washington, DC, USA), pp. 191-205, IEEE Computer Society, 2013.

[87] G. I. Apecechea, T. Eisenbarth, and B. Sunar, "Systematic Reverse Engineering of Cache Slice Selection in Intel Processors," IACR Cryptology ePrint Archive, vol. 2015, p. 690, 2015.

[88] M. K. Qureshi, A. Jaleel, Y. N. Patt, S. C. S. Jr., and J. S. Emer, "Adaptive insertion policies for high performance caching," in 34th International Symposium on Computer Architecture (ISCA 2007), June 9-13, 2007, San Diego, California, USA, pp. 381-391, 2007.

[89] D. Trilla, C. Hernández, J. Abella, and F. J. Cazorla, "Cache side-channel attacks and time-predictability in high-performance critical real-time systems," in Proceedings of the 55th Annual Design Automation Conference, DAC 2018, San Francisco, CA, USA, June 24-29, 2018, pp. 98:1-98:6, 2018.

[90] M. K. Qureshi, "CEASER: Mitigating Conflict-Based Cache Attacks via Encrypted-Address and Remapping," in IEEE/ACM International Symposium on Microarchitecture - MICRO 2018, 2018.

[91] R. Wilhelm, J. Engblom, A. Ermedahl, N. Holsti, S. Thesing, D. B. Whalley, G. Bernat, C. Ferdinand, R. Heckmann, T. Mitra, F. Mueller, I. Puaut, P. P. 
Puschner, J. Staschulat, and P. Stenström, "The worst-case execution-time problem - overview of methods and survey of tools," ACM Trans. Embedded Comput. Syst., vol. 7, no. 3, pp. 36:1-36:53, 2008.

[92] G. Doychev, B. Köpf, L. Mauborgne, and J. Reineke, "CacheAudit: A Tool for the Static Analysis of Cache Side Channels," ACM Trans. Inf. Syst. Secur., vol. 18, no. 1, pp. 4:1-4:32, 2015.

[93] R. Brotzman, S. Liu, D. Zhang, G. Tan, and M. Kandemir, "Casym: Cache aware symbolic execution for side channel detection and mitigation," in CaSym: Cache Aware Symbolic Execution for Side Channel Detection and Mitigation, p. 0, IEEE, 2019.

[94] A. Abel and J. Reineke, "uops.info: Characterizing latency, throughput, and port usage of instructions on intel microarchitectures," in Proceedings of the TwentyFourth International Conference on Architectural Support for Programming Languages and Operating Systems, ASPLOS '19, pp. 673-686, ACM, 2019.

[95] P. Pessl, D. Gruss, C. Maurice, M. Schwarz, and S. Mangard, "DRAMA: Exploiting DRAM addressing for cross-cpu attacks," in 25th USENIX Security Symposium (USENIX Security 16), (Austin, TX), pp. 565-581, USENIX Association, Aug. 2016.

[96] G. Rueda, "Learning cache replacement policies using register automata," 2013. Master's thesis, Uppsala University, Department of Information Technology.

[97] A. Abel and J. Reineke, "Measurement-based modeling of the cache replacement policy," in 19th IEEE Real-Time and Embedded Technology and Applications Symposium, RTAS 2013, Philadelphia, PA, USA, April 9-11, 2013, pp. 65-74, IEEE Computer Society, 2013.

[98] S. Briongos, P. Malagon, J. M. Moya, and T. Eisenbarth, "Reload+refresh: Abusing cache replacement policies to perform stealthy cache attacks," in 29th USENIX Security Symposium (USENIX Security 20), (Boston, MA), USENIX Association, Aug. 2020.

[99] A. Abel and J. Reineke, "Reverse engineering of cache replacement policies in intel microprocessors and their evaluation," in 2014 IEEE International Symposium on Performance Analysis of Systems and Software, ISPASS 2014, Monterey, CA, USA, March 23-25, 2014, pp. 141-142, 2014.

[100] AbsInt, "AbsInt aiT Worst-Case Execution Time Analyzers."

[101] N. Binkert, B. Beckmann, G. Black, S. K. Reinhardt, A. Saidi, A. Basu, J. Hestness, D. R. Hower, T. Krishna, S. Sardashti, et al., "The gem5 simulator," ACM SIGARCH computer architecture news, vol. 39, no. 2, pp. 1-7, 2011.

[102] J. Handy, The Cache Memory Book. San Diego, CA, USA: Academic Press Professional, Inc., 1993. 
[103] A. Malamy, R. Patel N., and N. M. Hayes, "Methods and apparatus for implementing a pseudo-lru cache memory replacement scheme with a locking feature," April 1992. US5353425A.

[104] M. Isberner, F. Howar, and B. Steffen, "The open-source learnlib," in Computer Aided Verification, Springer, 2015.

[105] A. Solar-Lezama, "The sketching approach to program synthesis," in Programming Languages and Systems, pp. 4-13, Springer Berlin Heidelberg, 2009.

[106] M. K. Qureshi, A. Jaleel, Y. N. Patt, S. C. Steely, and J. Emer, "Adaptive insertion policies for high performance caching," in Proceedings of the 34th Annual International Symposium on Computer Architecture, ISCA '07, pp. 381-391, ACM, 2007.

[107] P. Cañones, B. Köpf, and J. Reineke, "On the Incomparability of Cache Algorithms in Terms of Timing Leakage," Logical Methods in Computer Science, vol. Volume 15, Issue 1, 2019.

[108] D. Angluin, "Learning regular sets from queries and counterexamples," Inf. Comput., vol. 75, no. 2, pp. 87-106, 1987.

[109] O. Niese, An Integrated Approach to Testing Complex Systems. PhD thesis, Universität Dortmund, 2003.

[110] J. Moerman, Nominal Techniques and Black Box Testing for Automata Learning. PhD thesis, Radboud University, 2019.

[111] F. B. Khendek, S. Fujiwara, G. V. Bochmann, F. Khendek, M. Amalou, and A. Ghedamsi, "Test selection based on finite state models," IEEE Transactions on Software Engineering, vol. 17, pp. 591-603, 1991.

[112] Intel, "How to Benchmark Code Execution Times on Intel@ IA-32 and IA-64 Instruction Set Architectures," September 2010.

[113] E. Tromer, D. A. Osvik, and A. Shamir, "Efficient cache attacks on aes, and countermeasures," J. Cryptol., vol. 23, pp. 37-71, Jan. 2010.

[114] Intel, "Are noisy neighbors in your data center keeping you up at night," 2017.

[115] A. Abel and J. Reineke, "nanobench: A low-overhead tool for running microbenchmarks on x86 systems," Apr 2020.

[116] V. Kiriansky, I. A. Lebedev, S. P. Amarasinghe, S. Devadas, and J. S. Emer, "DAWG: A defense against cache timing attacks in speculative execution processors," in 51st Annual IEEE/ACM International Symposium on Microarchitecture, MICRO 2018, Fukuoka, Japan, October 20-24, 2018, pp. 974-987, 2018.

[117] F. Vaandrager, "Model Learning," Commun. ACM, vol. 60, no. 2, pp. 86-95, 2017. 
[118] W. Xiong and J. Szefer, "Leaking information through cache LRU states," CoRR, vol. abs/1905.08348, 2019.

[119] P. Koppe, B. Kollenda, M. Fyrbiak, C. Kison, R. Gawlik, C. Paar, and T. Holz, "Reverse Engineering x86 Processor Microcode," in Proceedings of the 26th USENIX Conference on Security Symposium, SEC'17, pp. 1163-1180, USENIX Association, 2017.

[120] B. Falk, "Sushi Roll: A CPU research kernel with minimal noise for cycle-by-cycle micro-architectural introspection," August 2019.

[121] C. Reis, A. Moshchuk, and N. Oskov, "Site isolation: Process separation for web sites within the browser," in 28th USENIX Security Symposium (USENIX Security 19), (Santa Clara, CA), pp. 1661-1678, USENIX Association, Aug. 2019.

[122] M. West, "Same-site cookies." https://tools.ietf.org/html/ draft-west-first-party-cookies-07, 2016.

[123] S. Cassel, F. Howar, B. Jonsson, and B. Steffen, Extending Automata Learning to Extended Finite State Machines, pp. 149-177. Springer International Publishing, 2018.

[124] A. Khalili and A. Tacchella, "Learning nondeterministic mealy machines," in The 12th International Conference on Grammatical Inference (A. Clark, M. Kanazawa, and R. Yoshinaka, eds.), vol. 34 of Proceedings of Machine Learning Research, (Kyoto, Japan), pp. 109-123, PMLR, 17-19 Sep 2014.

[125] S. Cai, M. Jahangoshahi, M. Bakshi, and S. Jaggi, "Efficient algorithms for noisy group testing," IEEE Transactions on Information Theory, vol. 63, pp. 21132136, April 2017. 